\title{
Identification of Novel Biomarkers for Predicting Prognosis and Immunotherapy Response in Head and Neck Squamous Cell Carcinoma Based on ceRNA Network and Immune Infiltration Analysis
}

\author{
Ya Guo $\mathbb{D}^{1}$, Wei Kang Pan $\mathbb{D}^{2},{ }^{2}$ Zhong Wei Wang $\mathbb{D}^{1},{ }^{1}$ Wang Hui Su ${ }^{D},{ }^{1}$ Kun Xu $\mathbb{D}^{1}$, \\ Hui Jia $\mathbb{D}^{1}$, and Jing Chen $\mathbb{D}^{1}$ \\ ${ }^{1}$ Department of Radiation Oncology, The Second Affiliated Hospital, Xi'an Jiao Tong University, Xi'an, 710004 Shaanxi, China \\ ${ }^{2}$ Department of Pediatric Surgery, The Second Affiliated Hospital, Xi'an Jiao Tong University, Xi'an, 710004 Shaanxi, China
}

Correspondence should be addressed to Ya Guo; gy8569851@163.com

Received 3 September 2021; Revised 23 October 2021; Accepted 19 November 2021; Published 6 December 2021

Academic Editor: Rui Liu

Copyright @ 2021 Ya Guo et al. This is an open access article distributed under the Creative Commons Attribution License, which permits unrestricted use, distribution, and reproduction in any medium, provided the original work is properly cited.

\begin{abstract}
Objectives. Patients with head and neck squamous cell carcinoma (HNSCC) have poor prognosis and show poor responses to immune checkpoint (IC) inhibitor (ICI) therapy. Competing endogenous RNA (ceRNA) networks, tumor-infiltrating immune cells (TIICs), and ICIs may influence tumor prognosis and response rates to ICI therapy. This study is aimed at identifying prognostic and IC-related biomarkers and key TIIC signatures to improve prognosis and ICI therapy response in HNSCC patients. Methods and Results. Ninety-five long noncoding RNAs (lncRNAs), microRNAs (miRNAs), and 1746 mRNAs were identified using three independent methods. We constructed a ceRNA network and estimated the proportions of 22 immune cell subtypes. Ten ceRNAs were related to prognosis according to Kaplan-Meier analysis. Two risk signatures based, respectively, on nine ceRNAs (ANLN, CFL2, ITGA5, KDELC1, KIF23, NFIA, PTX3, RELT, and TMC7) and three immune cell types (naïve B cells, neutrophils, and regulatory $\mathrm{T}$ cells) via univariate Cox regression, least absolute shrinkage and selection operator, and multivariate Cox regression analyses could accurately and independently predict the prognosis of HNSCC patients. Key mRNAs in the ceRNA network were significantly correlated with naïve B cells and regulatory $\mathrm{T}$ cells and with stage, grade, and immune and molecular subtype. Eight IC genes exhibited higher expression in tumor tissues and were correlated with eight key mRNAs in the ceRNA network in HNSCC patients with different HPV statuses according to coexpression and TIMER 2.0 analyses. Most drugs were effective in association with expression of these key signatures (ANLN, CFL2, ITGA5, KIF23, NFIA, PTX3, RELT, and TMC7) based on GSCALite analysis. The prognostic value of key biomarkers and associations between key ceRNAs and IC genes were validated using online databases. Eight key ceRNAs were confirmed to predict response to ICI in other cancers based on TIDE analysis. Conclusions. We constructed two risk signatures to accurately predict prognosis in HNSCC. Key IC-related signatures may be associated with response to ICI therapy. Combinations of ICIs with inhibitors of eight key mRNAs may improve survival outcomes of HNSCC patients.
\end{abstract}

\section{Introduction}

Head and neck cancer (HNC) is among the most common malignancies worldwide, accounting for about 600,000 new cases annually and 330,000 deaths [1]. Head and neck squamous cell carcinoma (HNSCC) is the predominant pathological subtype, comprising more than $90 \%$ of HNC cases [2]. Despite advances in surgery and radiotherapy, the 5- year overall survival (OS) rate for HNSCC is still unsatisfactory $[3,4]$. Thus, there is an indisputably urgent need to identify effective biomarkers to predict prognosis and OS of patients with HNSCC.

Regulatory competing endogenous RNA (ceRNA) networks, consisting of long noncoding RNAs (lncRNAs), microRNAs (miRNAs), and mRNAs, have a crucial role in the processes of tumor occurrence and progression and in 
prediction of prognosis $[5,6]$. Furthermore, accumulating evidence suggests that immune cell infiltration may have a significant impact on the prognosis of HNSCC patients [7, 8]. Although the prognostic value of ceRNA networks and immune cell infiltration in HNSCC have been reported, few studies have combined ceRNA networks and immune cell infiltration to predict prognosis in HNSCC patients; moreover, these studies have limitations, and their conclusions have been inconsistent. Further, few studies have had their results successfully confirmed in another independent database, and in many, a single analytical method was used to explore key molecules or immune cells [5, 9-11]. Moreover, in HNSCC, multiple clinical characteristics, including human papillomavirus (HPV) status, immune subtype, molecular subtype, grade, and stage, are associated with prognosis $[12,13]$. There has not been sufficient systematic evaluation of the association among ceRNA-network RNAs, immune cell infiltration, and these clinical characteristics to fully elucidate the roles of these factors in prognosis. Therefore, a systematic scientific approach is needed to identify effective biomarkers for risk assessment of patient prognosis.

HNSCCs comprise a group of highly heterogeneous and immunosuppressive cancers.

Immunotherapy is aimed at increasing the activity of the immune system to eliminate cancer cells [14]. Immune checkpoint (IC) inhibitors (ICIs) represent a broadly effective immunotherapy that can block inhibitory IC pathways to restore antitumor immunity.

Anti-PD1/PDL1 ICIs can improve antitumor immune activity [4]. Although ICIs exert beneficial therapeutic effects on HNSCC, the response rate to them is still low [15]. Therefore, identifying biomarkers able to predict ICI treatment response can contribute to patient screening and individualized treatment approaches and is of great significance for standardizing immunosuppressive therapy and improving the prognosis of HNSCC patients. Previous studies have revealed that the expression of immunosuppressive molecules and tumor-infiltrating immune cells (TIICs) may regulate the response rate to immunotherapy and prognosis in HNSCC patients. A previous study proposed the epithelial-mesenchymal transition CYT Index as a superior predictor of prognosis and immunotherapy response across HNSCC [16], and immune cell infiltration scores were identified as sensitive prognostic biomarkers and predictive indicators for immunotherapy response [8]. However, no reliable biomarker for predicting response to ICIs and prognosis in HNSCC has yet been identified $[17,18]$.

In this study, mRNA, IncRNA, and miRNA expression profiles of HNSCC were downloaded from The Cancer Genome Atlas (TCGA) database to construct a ceRNA network. CIBERSORT (cell type identification by estimating relative subset of known RNA transcripts) was used to estimate the abundance of 22 immune cells based on TCGA dataset. Furthermore, two prognostic signatures were developed using a comprehensive bioinformatics method. Next, the association between key genes and immune cells was evaluated via coexpression analysis. We systematically investigated the associations between key ceRNA signatures and clinical characteristic (including stage, grade, and immune and molecular subtype). Moreover, IC correlation analysis and key ceRNA-related drug targets were performed to assess the predictive ability of these biomarkers with respect to ICI treatment response in HNSCC and to explore potential targets for improving the effectiveness of immunotherapy. Finally, the prognostic value of key biomarkers and the associations between key ceRNAs and IC genes were validated using online databases.

\section{Materials and Methods}

2.1. Data Analysis and ceRNA Network Construction. RNA sequencing (RNA-seq) and miRNA-seq data and clinical information for HNSCC patients were extracted from TCGA database (https://gdc-portal.nci.nih.gov/) [19]. HTseq-count profiles were obtained. In addition, practical extraction and reports Language (Perl) scripts were used to merge all clinical data. The $\mathrm{R}$ software (version 3.6.3, https://www.r-project .org/) was used to process downloaded files, to convert and eliminate unqualified data, to apply a relatively conservative approach to the data analysis, and to identify a suitable number of differentially expressed mRNAs (DEmRNAs), miRNAs (DEmiRNAs), and lncRNAs (DElncRNAs). The limma, edgeR, and DESeq2 R packages were used for differential expression analysis. Log fold change $>1$ or $<-1$ and false discovery rate- (FDR-) adjusted $P$ value $<0.05$ were used as the thresholds to identify significantly expressed biomarkers. Only biomarkers that were identified using three independent analysis methods were selected as the final DEmRNAs, DEmiRNAs, and DElncRNAs [20]. A ceRNA network was established using the "GDCRNATools" $\mathrm{R}$ package. The ceRNA network was evaluated by hypergeometric testing and correlation analysis. $P<0.05$ was considered to indicate a statistically significant result [21]. Finally, Cytoscape v.3.7.2 was used to visualize the ceRNA network [22].

2.2. Construction and Confirmation of ceRNA-Related Prognostic Model. We performed univariate Cox regression, least absolute shrinkage and selection operator (LASSO), and multivariate Cox regression analyses to identify prognosis-related signatures. Moreover, we plotted receiver operating characteristic (ROC) curves for 1-year, 3-year, and 5-year survival and calculated the corresponding area under the curve (AUC) values using the survival and time ROC packages to assess the predictive ability of the ceRNArelated signatures [23]. An AUC of $<0.5$ was considered insignificant. Patients were then divided into high- and low-risk groups according to the average risk score. We further calculated survival differences between the high- and low-risk groups [24]. Next, we performed univariate and multivariate Cox regression analyses to determine whether the risk score based on key ceRNAs was independent of other clinical characteristics (age, sex, grade, and stage) in the prediction of HNSCC prognosis. A hazard ratio $(\mathrm{HR})>1$ and $P<0.05$ were considered to indicate unfavorable prognostic factors, whereas $\mathrm{HR}<1$ and $P<0.05$ indicated favorable prognostic factors [25]. 


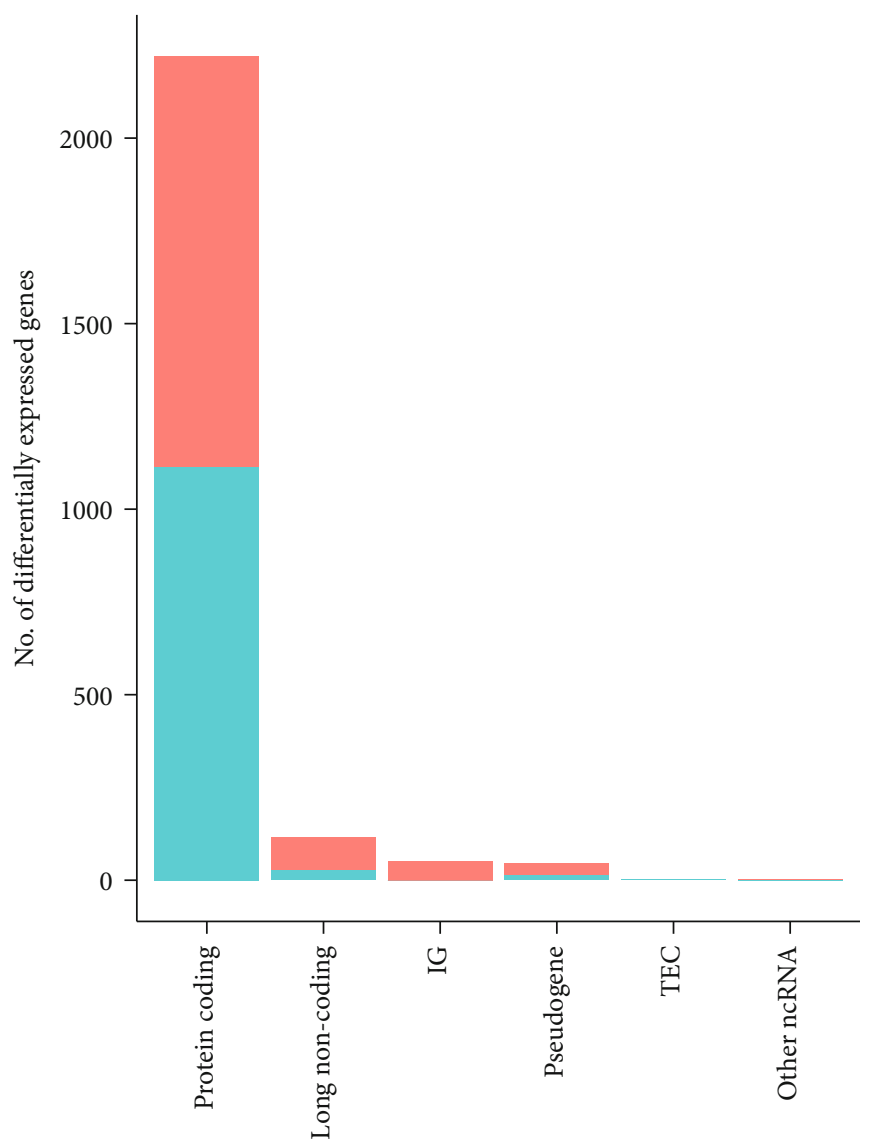

Up-regulated

Down-regulated

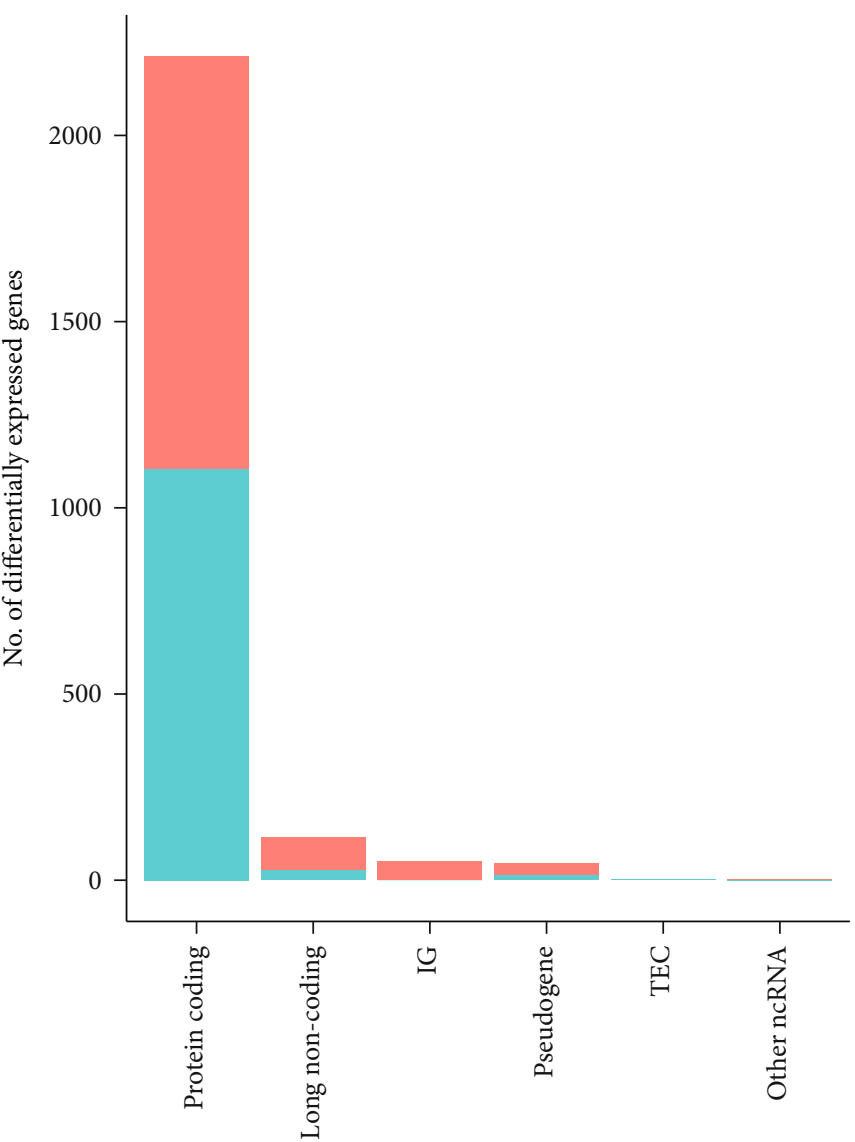

Up-regulated

Down-regulated

(a)

(b)

FIgUre 1: Continued. 


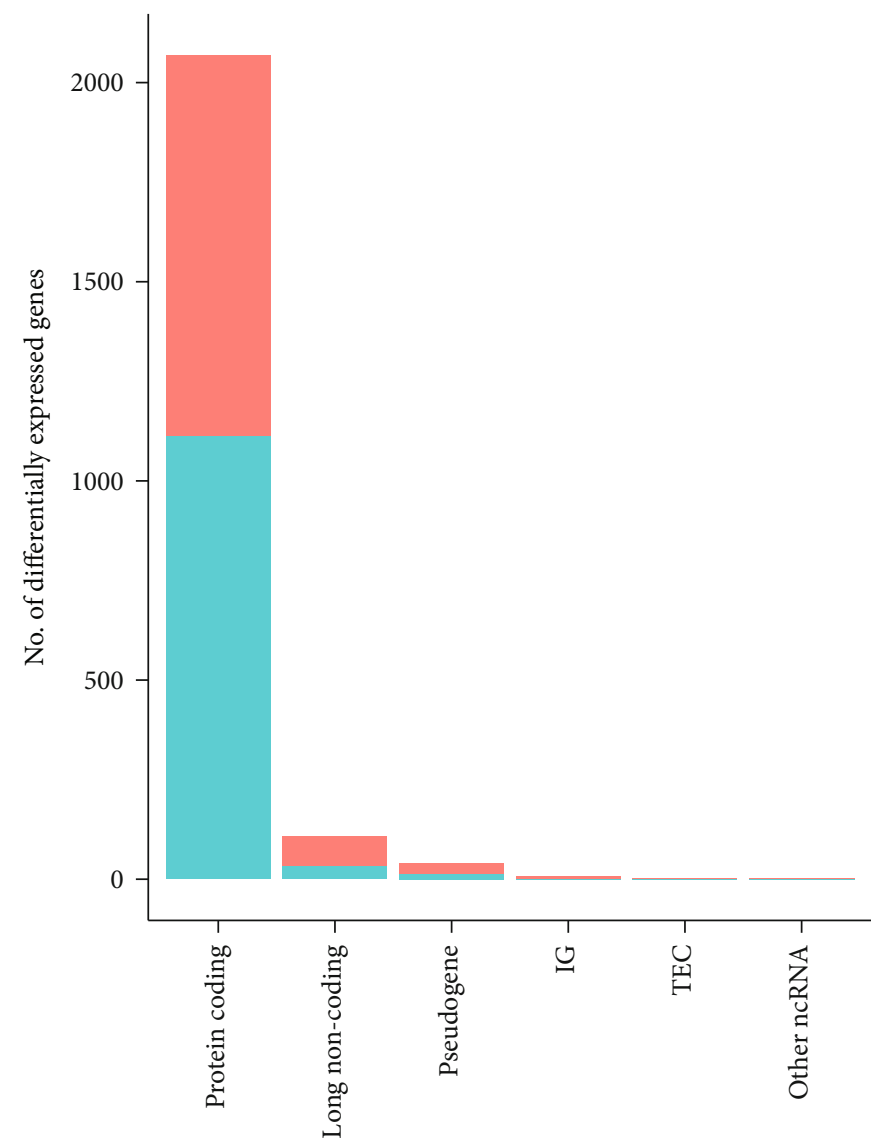

Up-regulated

Down-regulated

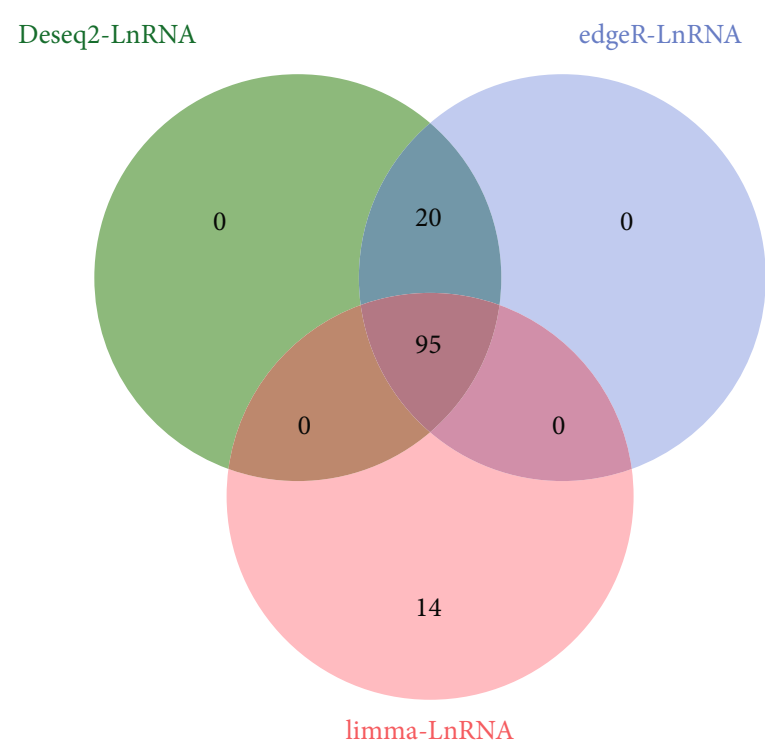

Size of each list

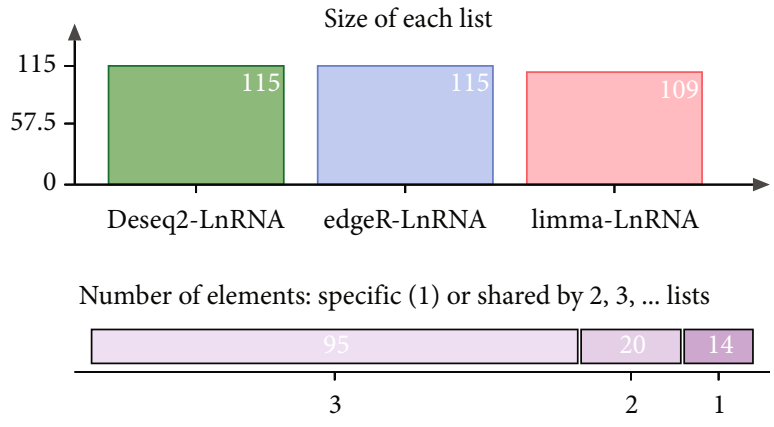

(d)

FIgUre 1: Continued. 

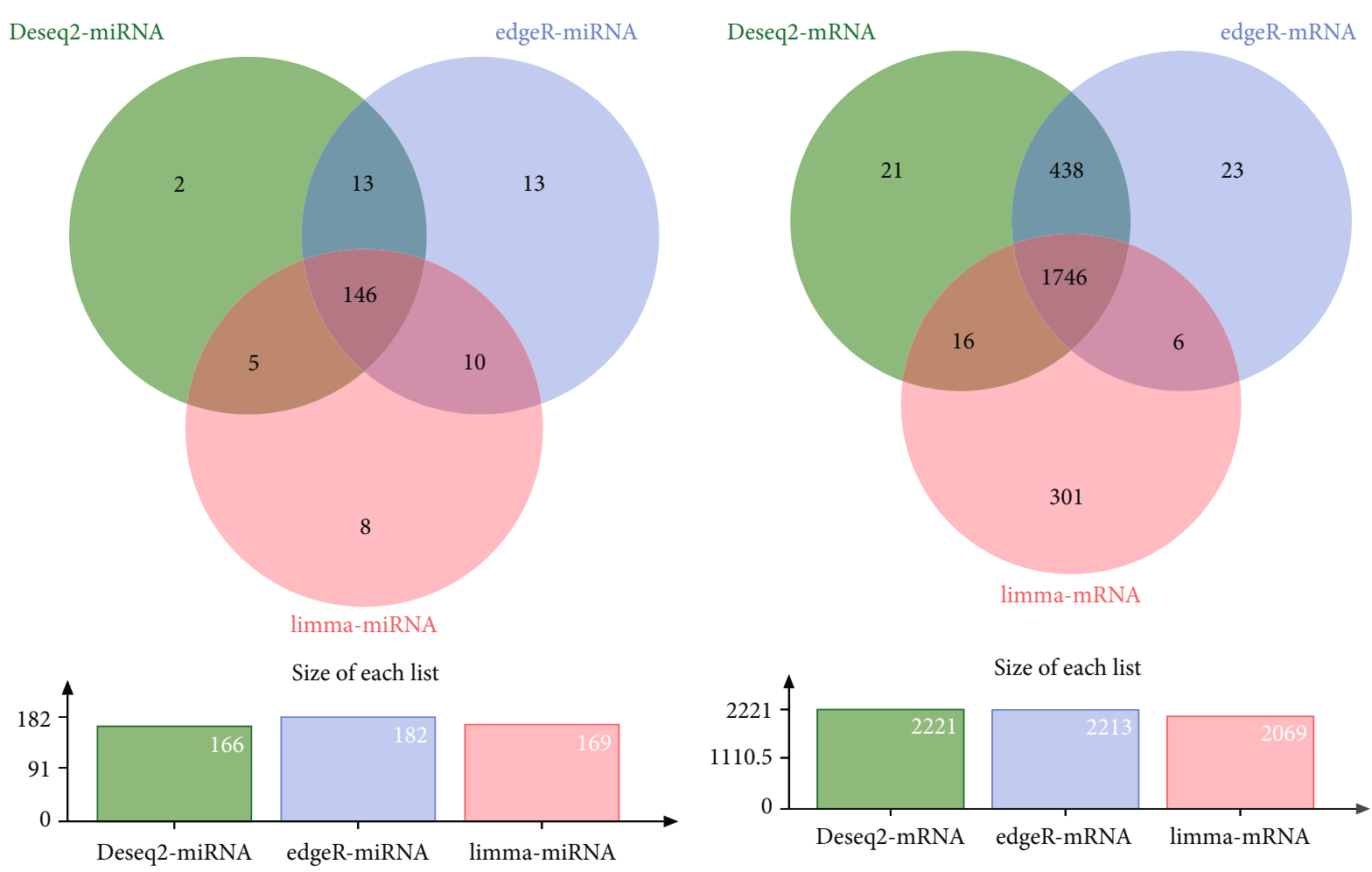

Number of elements: specific (1) or shared by $2,3, \ldots$ lists

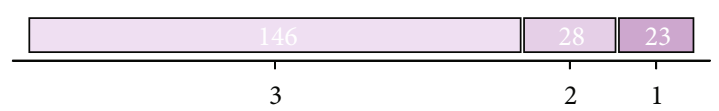

Number of elements: specific (1) or shared by $2,3, \ldots$ lists

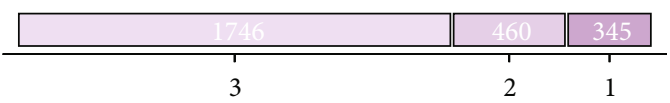

(e)

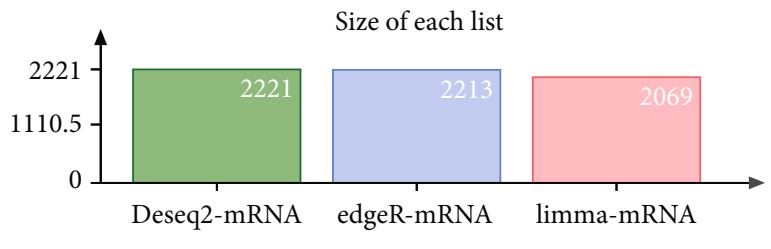

(f)

Figure 1: Continued. 


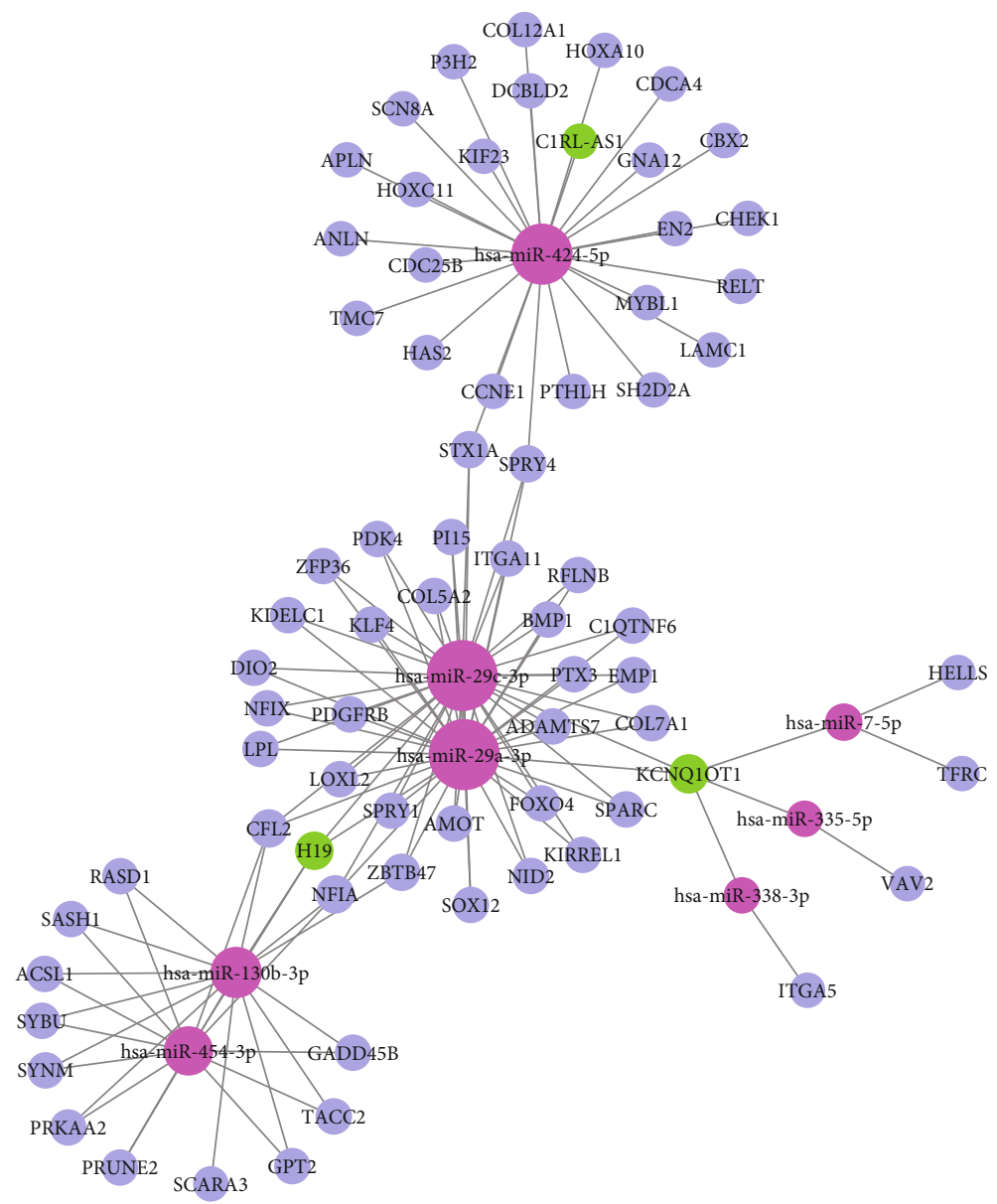

(g)

FIGURE 1: Identification of DElncRNAs, DEmiRNAs, DEmRNAs, and construction of the ceRNA network. Differentially expressed genes were identified by three independent methods. (a) Deseq2, (b) edgeR, and (c) limma. (d-f) Venn diagram displaying the intersection of (d) DElncRNAs, (e) DEmiRNAs, and (f) DEmRNAs. (g) Construction of the ceRNA network. Red circles represent miRNAs, green circles represent lncRNAs, and purple circles represent mRNAs. TEC: to be experimentally confirmed; IG: immunoglobulin. Log fold change $>1$ or $<-1$ and FDR-adjusted $P$ value $<0.05$ were used to identify significantly expressed biomarkers.

2.3. Construction of Immune Cell-Related Prognostic Model. The CIBERSORT algorithm was used to estimate the abundance of 22 immune cell subtypes according to the RNAseq count data [26]. The associations between immune cell types and prognosis were investigated by univariate Cox regression, LASSO regression, and multivariate Cox regression. We further assessed the sensitivity and specificity of immune cell-related prognostic models using ROC curves. In addition, we performed an independent prognostic analysis to assess whether the risk score based on key immune cells could predict HNSCC prognosis independently of other clinical characteristics.

2.4. Associations between Key ceRNAs and Significant Immune Cell Subtypes. The associations between significant mRNAs in the ceRNA network and key immune cells were investigated using Pearson correlation coefficients. Moreover, we analyzed the associations between the gene risk score and key immune cells.
2.5. Clinical Correlation Analysis. The Tumor Immune System Interactions Database (TISIDB; http://cis.hku.hk/ TISIDB) was used to explore Spearman correlations between the identified key ceRNAs and clinical characteristics. Moreover, the expression distribution patterns of nine key ceRNAs across immune and molecular subtypes were determined using TISIDB [27]. The immune subtypes comprised five groups: C1 (wound healing), C2 (IFN-gamma dominant), C3 (inflammatory), C4 (lymphocyte depleted), and C6 (TGF- $\beta$ dominant) [12]. HNSCC can be classified into four molecular subtypes, namely, atypical (AT), basal (BA), classical (CL), and mesenchymal (MS) [13].

2.6. Correlations between Immunotherapy Response and Key ceRNAs. The expression of ICs is correlated with the immune response to immunotherapy. We extracted the expression levels of eight immune-checkpoint genes (CD274, CTLA4, HAVCR2, LAG3, PDCD1, PDCD1LG2, 

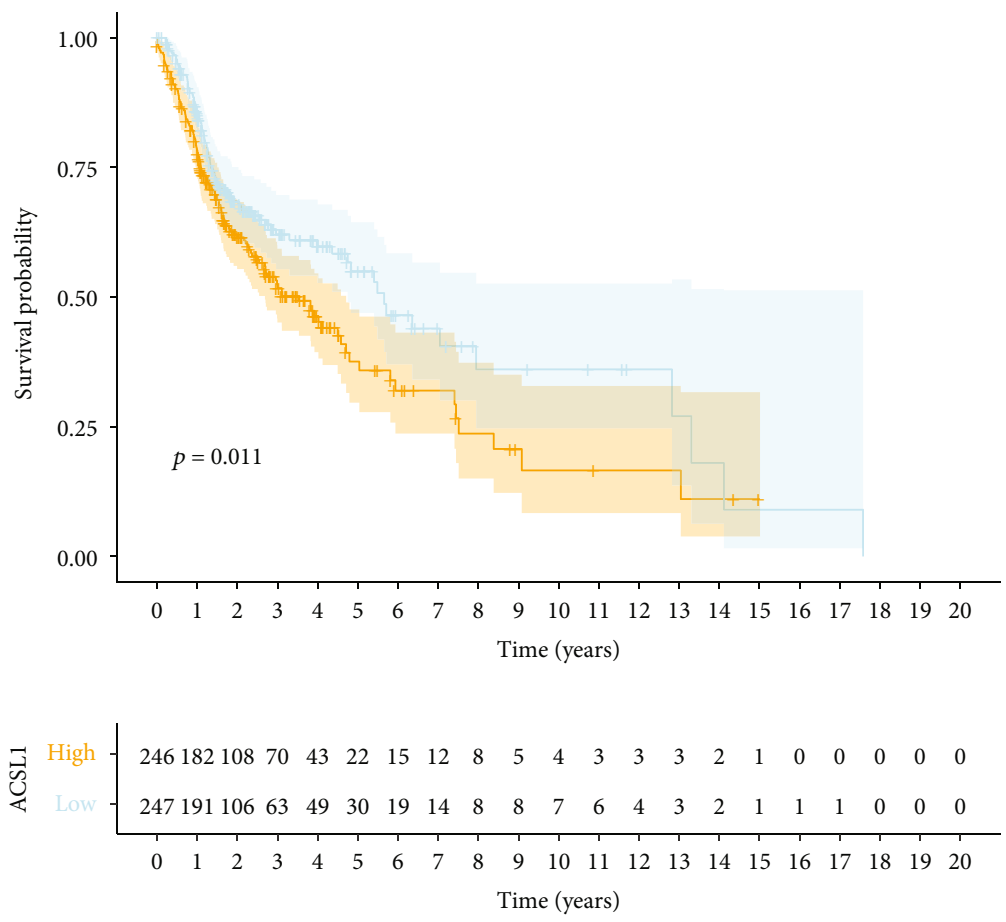

ACSL1

+ High

Low

(a)
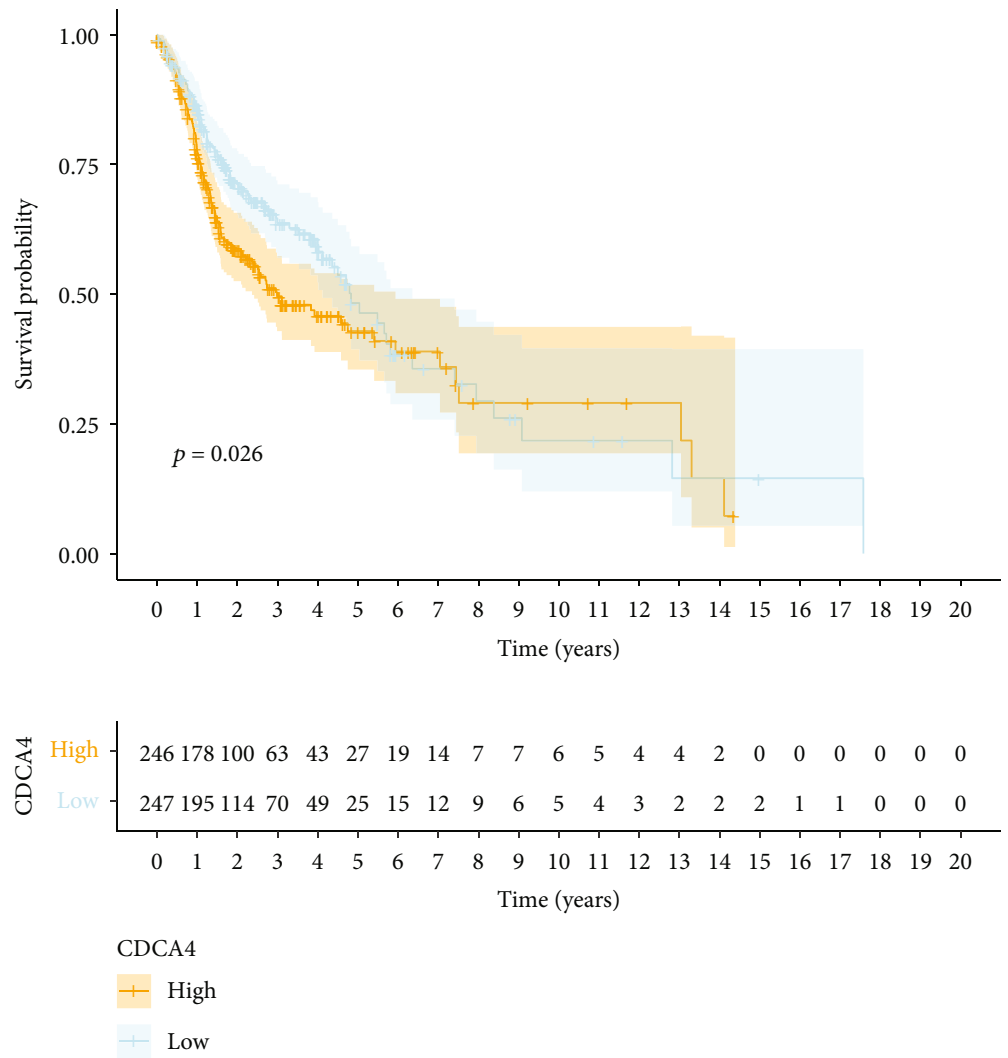

(b)

Figure 2: Continued. 

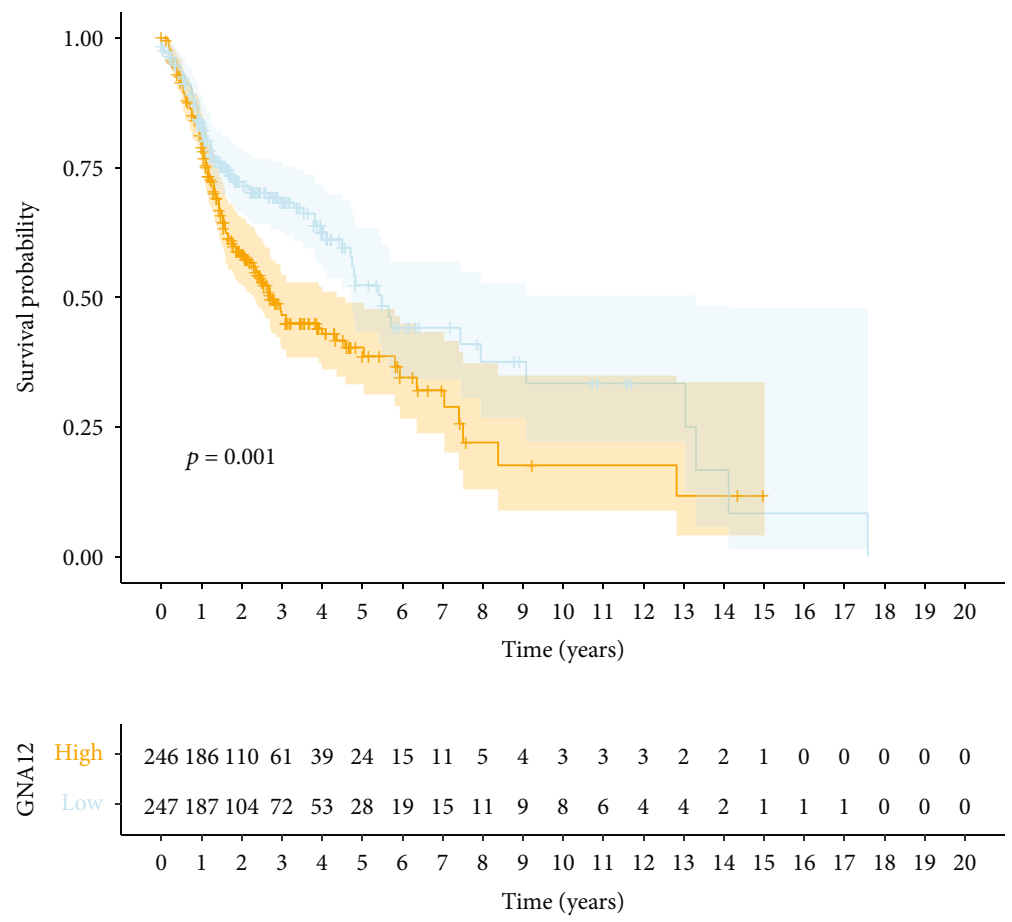

GNA12

+ High
$+\quad$ Low

(c)

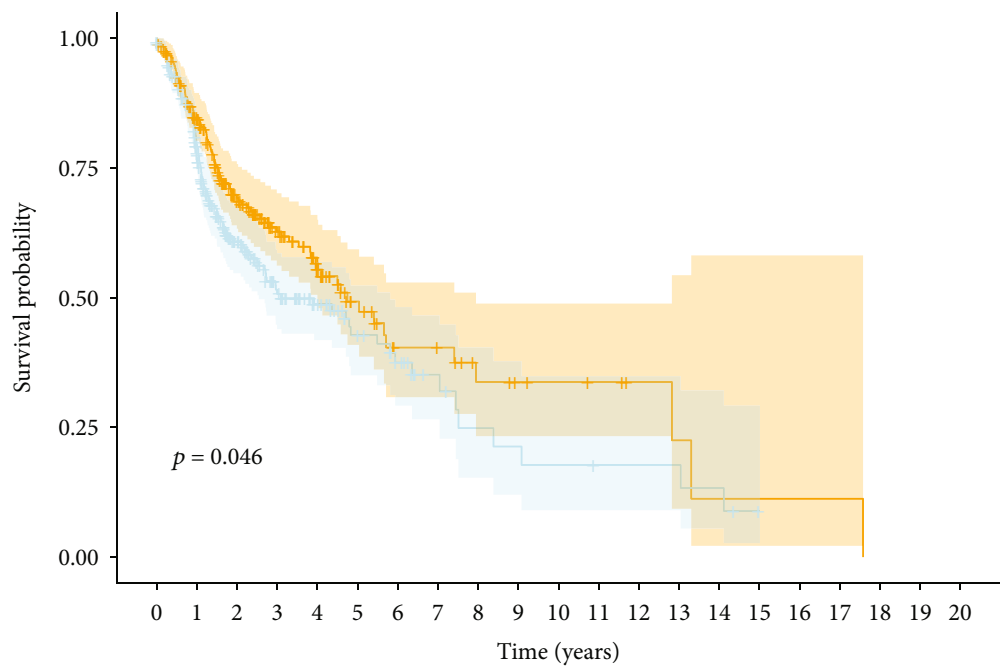

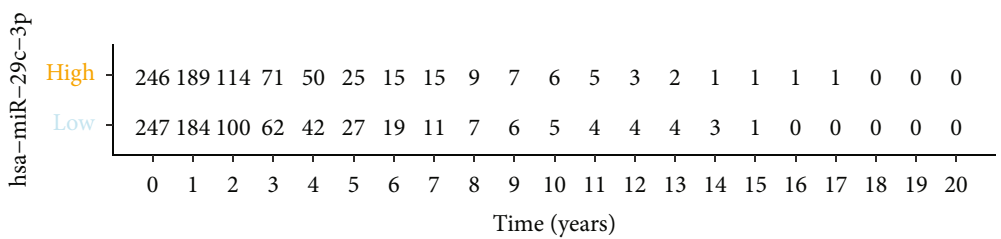

hsa $-\mathrm{miR}-29 \mathrm{c}-3 \mathrm{p}$

+ High

Low

(d)

Figure 2: Continued. 

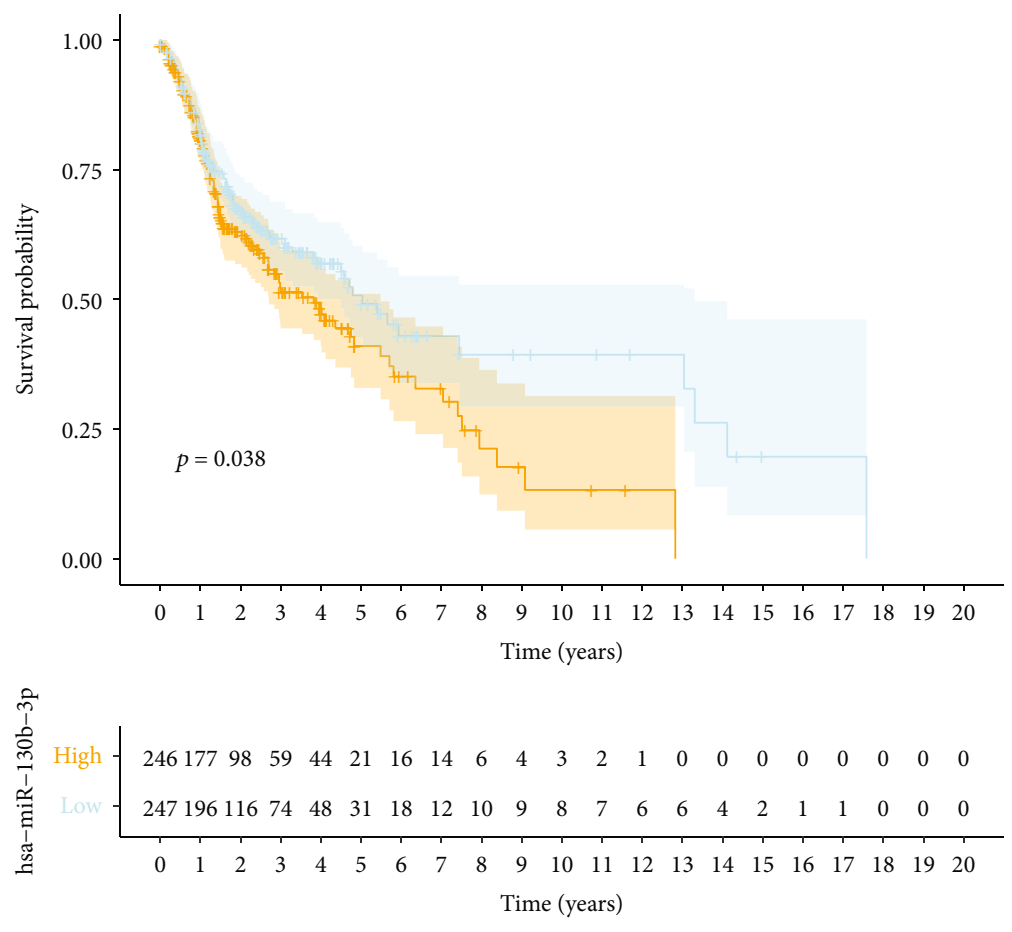

hsa - miR-130b-3p

+ High

+ Low

(e)

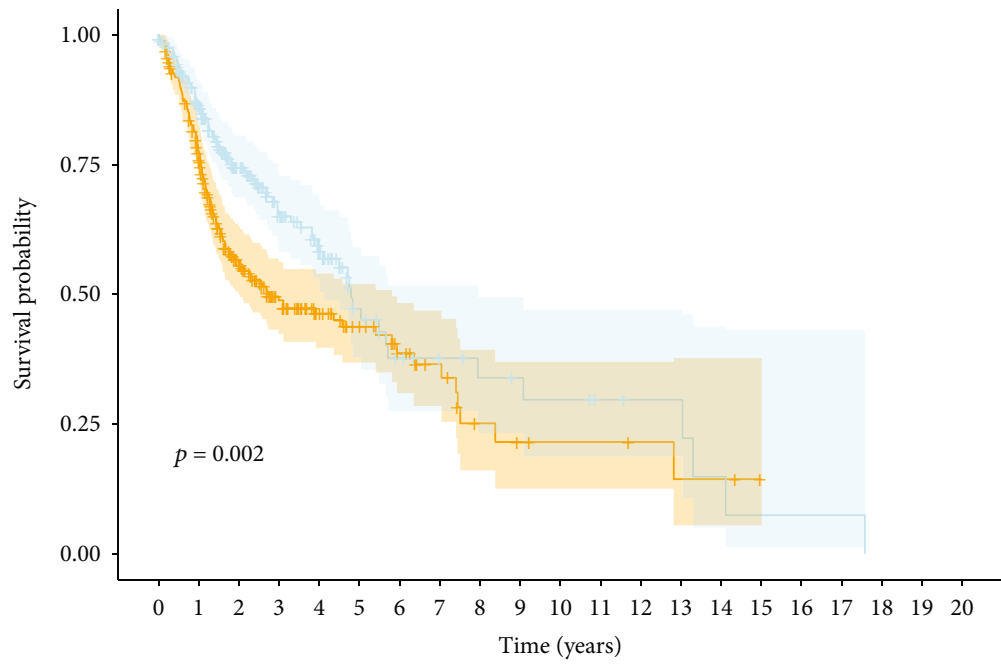

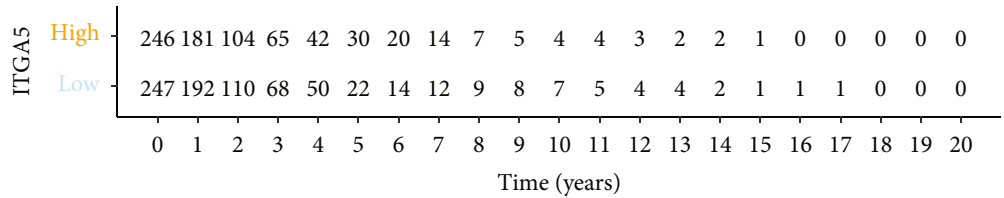

ITGA5

+ High

Low

(f)

Figure 2: Continued. 

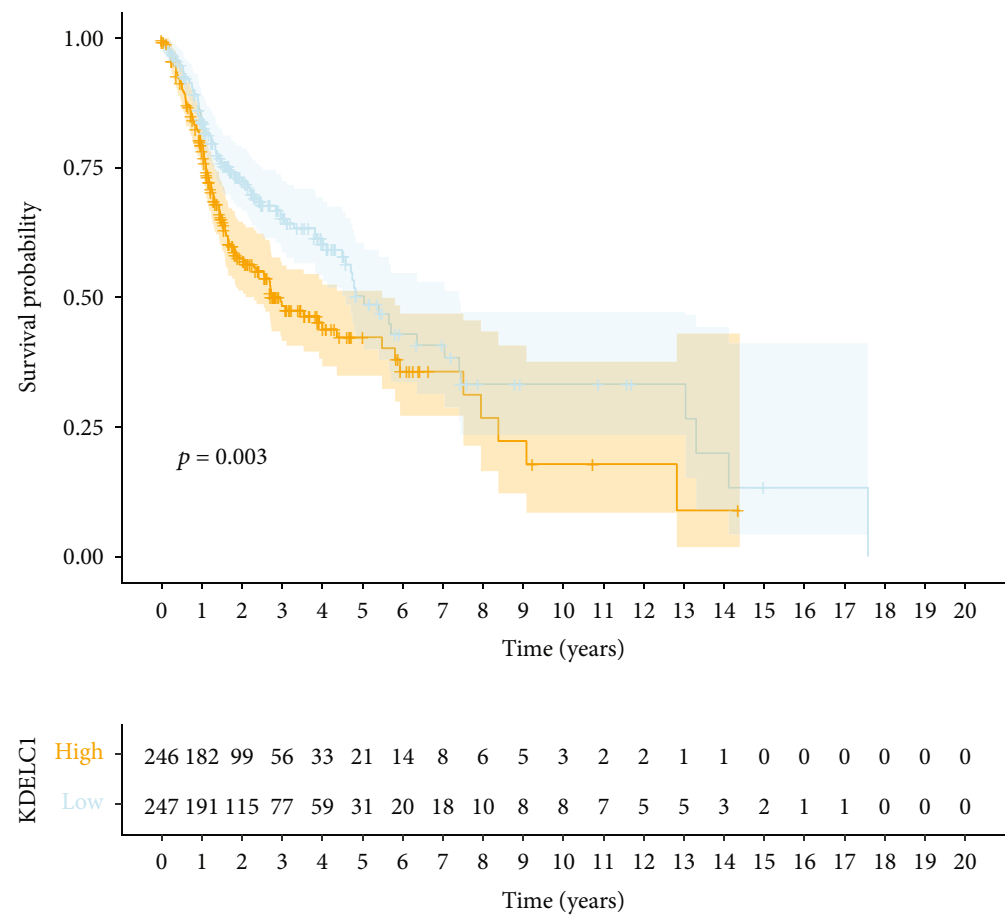

KDELC1

+ High

+ Low

(g)

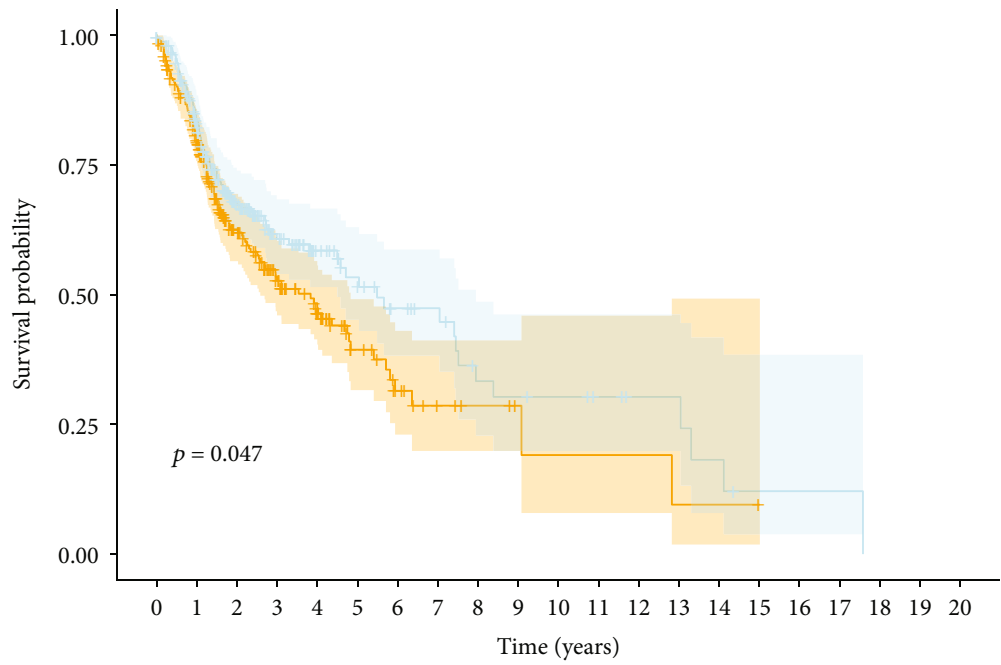

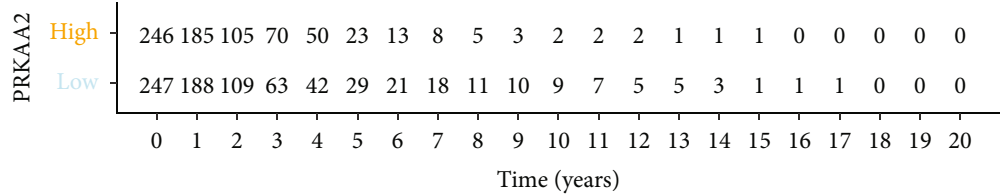

PRKAA2

+ High

Low

(h)

Figure 2: Continued. 

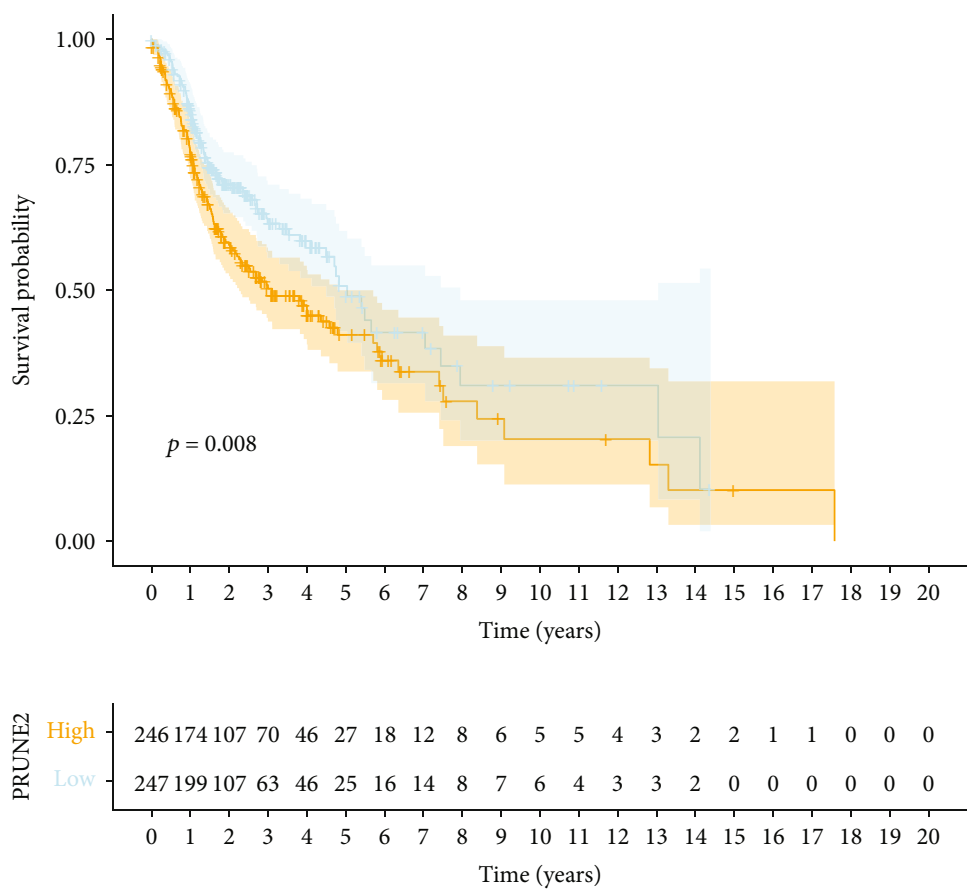

PRUNE2

+ High

Low

(i)
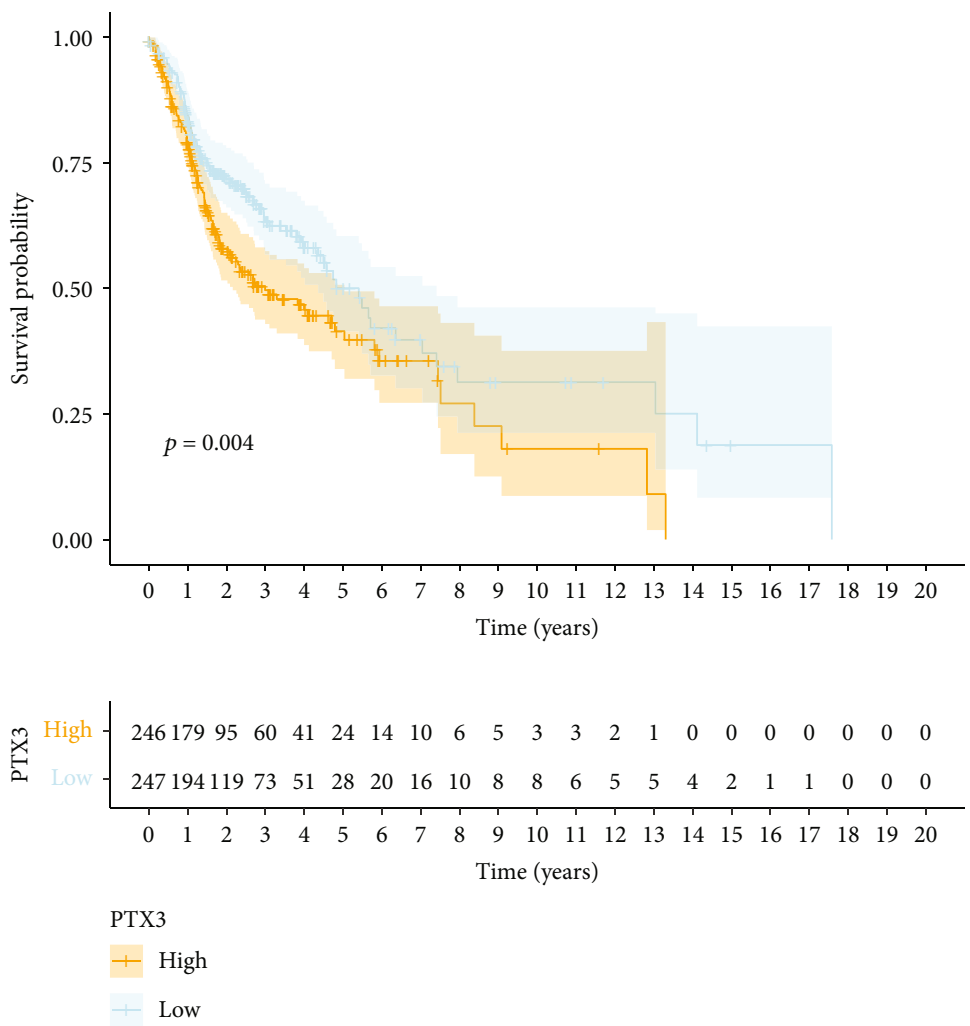

(j)

Figure 2: Survival analysis of key members in the ceRNA network. (a-j) K-M survival curves for the significant members of the ceRNA network. (a) ACSL1, (b) CDCA4, (c) GNA12, (d) hsa-miR-29c-3p, (e) hsa-miR-130b-3p, (f) ITGA5, (g) KDELC1, (h) PRKAA2, (i) PRUNE2, and (j) PTX3. 
TABLE 1: Identification of prognosis-related ceRNAs by univariate Cox regression analysis.

\begin{tabular}{lcccc}
\hline Id & HR & HR.95L & HR.95H & P value \\
\hline ANLN & 1.2194 & 1.0487 & 1.4179 & 1.2617 \\
C1QTNF6 & 1.1279 & 1.0083 & 1.3756 & 0.0099 \\
CFL2 & 1.2049 & 1.0554 & 1.3166 & 0.0058 \\
DCBLD2 & 1.1560 & 1.0149 & 1.5816 & 0.0290 \\
GNA12 & 1.3001 & 1.0687 & 1.3567 & 0.0087 \\
ITGA5 & 1.2156 & 1.0892 & 1.5753 & 0.0005 \\
KDELC1 & 1.3339 & 1.1295 & 1.5034 & 0.0007 \\
KIF23 & 1.2437 & 1.0289 & 0.9319 \\
NFIA & 0.7776 & 0.6488 & 1.1292 & 0.0242 \\
PRUNE2 & 1.0667 & 1.0078 & 1.1882 & 0.0065 \\
PTX3 & 1.1174 & 1.0509 & 1.4822 & 0.0260 \\
RELT & 1.2274 & 1.0165 & 1.2926 \\
TMC7 & 1.1395 & 1.0046 & 0.0004 \\
\hline
\end{tabular}

TIGIT, and SIGLEC15) and compared their differences in expression between tumor and normal tissues in HNSCC using the $\mathrm{R}$ packages limma, ggplot2, and pheatmap. Coexpression analysis was used to define the correlations between the expression levels of nine key ceRNAs and those of the eight immune-checkpoint genes. A two-gene correlation map was obtained using the R package ggstatsplot. A $P$ value of less than 0.05 was considered to indicate statistical significance. The Tumor Immune Dysfunction and Exclusion (TIDE, http://tide.dfci.harvard.edu) algorithm was then used to estimate the predictive power of the custom biomarker with respect to response outcome and OS [28]. We confirmed the correlations between the eight key mRNAs and response to ICIs in other cancers using TIDE.

2.7. Correlations between Expression of Key ceRNAs and Drug Sensitivity. Gene Set Cancer Analysis (GSCALite, http://bioinfo.life.hust.edu.cn/web/GSCALite/) is a web server for dynamic analysis and visualization of gene sets in cancer and drug sensitivity correlations. The expression of each gene in the gene set was determined, and small molecule/drug sensitivity (half-maximal inhibitory concentration; IC50) was obtained using Spearman's correlation analysis. Correlations with $\mathrm{FDR}<0.05$ were considered to represent results [29-31]. We used GSCALite to analyze the correlations between drug sensitivity and the signature of nine key ceRNAs.

2.8. Validation of Results. LOGpc (Long-term Outcome and Gene Expression Profiling Database of pan-cancers, http:// bioinfo.henu.edu.cn/DatabaseList.jsp) includes 209 gene expression datasets and provides 13 types of survival terms for 31,310 patients with 27 distinct malignancies [32]. We first confirmed the prognostic value of major target genes using LOGpc datasets. Next, we confirmed the prognostic value of key immune cell types and ceRNAs using the TIMER 2.0 database [33]. Finally, we confirmed the expression of eight immune-checkpoint genes and determined the correlations between the expression levels of these genes and nine key genes in HNSCC with different HPV statuses using the gene correlation TIMER 2.0 module (http://timer. cistrome.org/) [34].

2.9. Statistical Analysis. All statistical analyses were carried out using $\mathrm{R}$ (version 3.6.3, https://www.r-project.org/) and the limma, GDCRNATools, ggplot2, rms, glmnet, survminer, and timeROC packages. A two-sided $P$ value of less than 0.05 was considered to indicate statistical significance.

\section{Results}

3.1. Identification of Differentially Expressed Genes, DEmiRNAs, and DElncRNAs. We identified 115 DElncRNAs, 166 DEmiRNAs, and 2221 DEmRNAs using the DESeq2 package in the $\mathrm{R}$ programming language. Of these, 115 lncRNAs, 182 miRNAs, and 2213 mRNAs were found to be differentially expressed in HNSCC using the edgeR method. Next, we acquired 109 DElncRNAs, 169 DEmiRNAs, and 2069 DEmRNAs using the R package limma. A total of 95 DElncRNAs, 146 DEmiRNAs, and 1746 DEmRNAs were identified using three independent methods (Figures 1(a)-1(f)).

3.2. Construction of ceRNA Network and Survival Analysis. We constructed a IncRNA-miRNA-mRNA ceRNA network, which included three lncRNAs, eight miRNAs, and 69 mRNAs (Figure 1(g)). Then, Kaplan-Meier (K-M) survival analysis was performed to identify prognostic members in the constructed ceRNA network. Our results showed that 10 key markers, including ACSL1, CDCA4, GNA12, hsamiR-29c-3p, hsa-miR-130b-3p, ITGA5, KDELC1, PRKAA2, PRUNE2, and PTX3, were significantly correlated with survival (Figures 2(a)-2(j)).

3.3. Construction of ceRNA-Related Prognostic Model. Thirteen ceRNAs were identified as prognosis-related signatures using univariate Cox regression (Table 1). LASSO regression analysis revealed that nine key markers were essential for modeling (Figures 3(a) and 3(b)); these were subjected to 


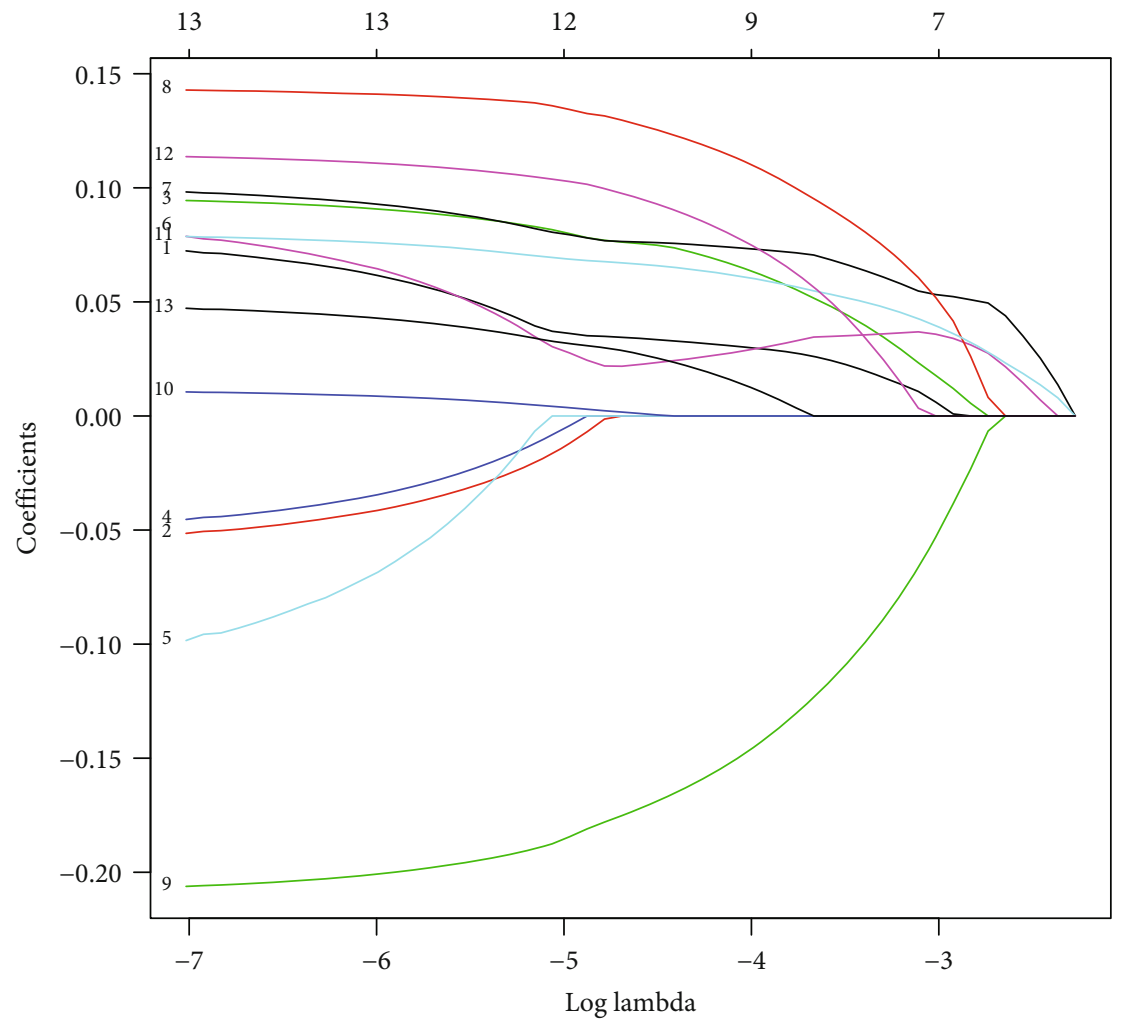

(a)

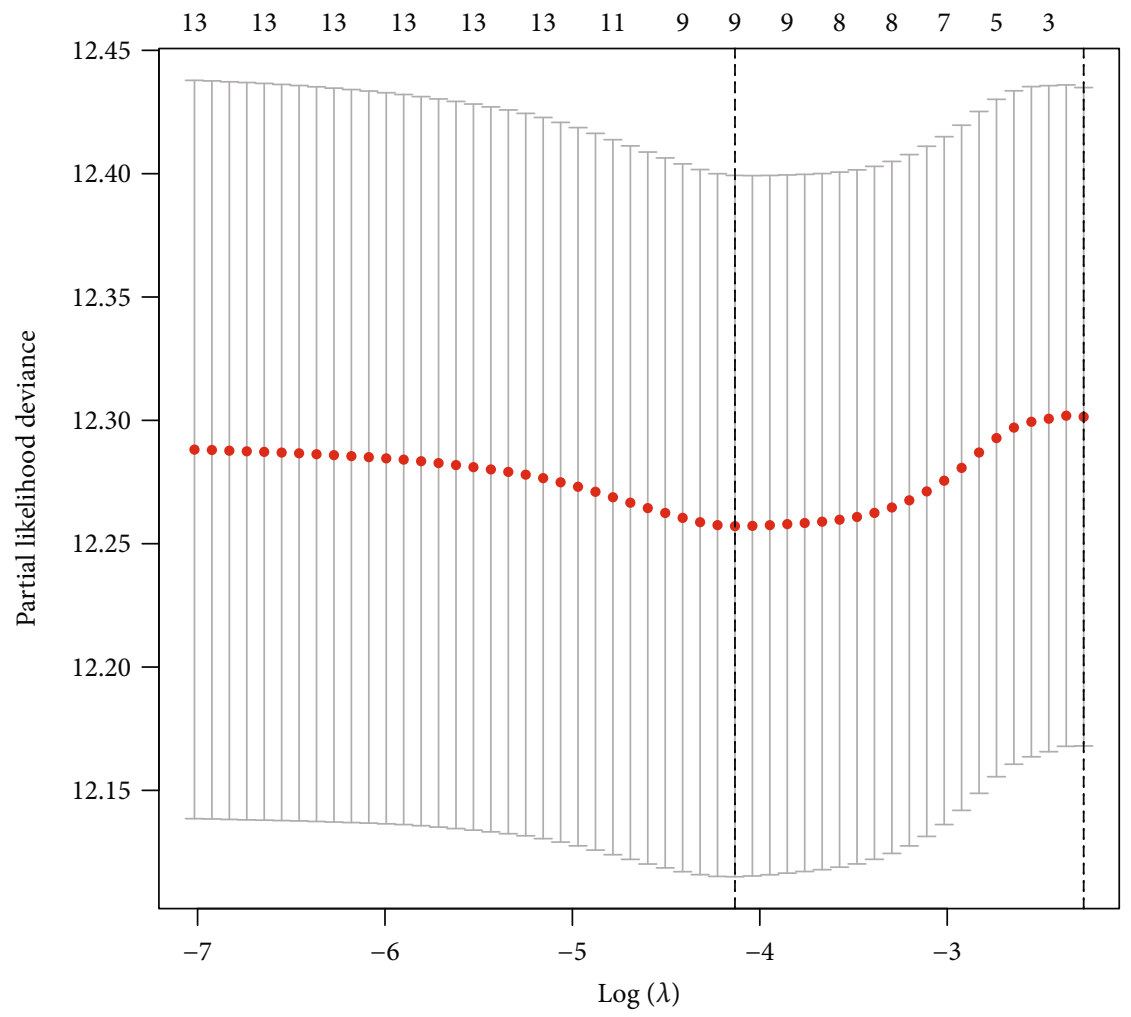

(b)

FIgUre 3: Continued. 


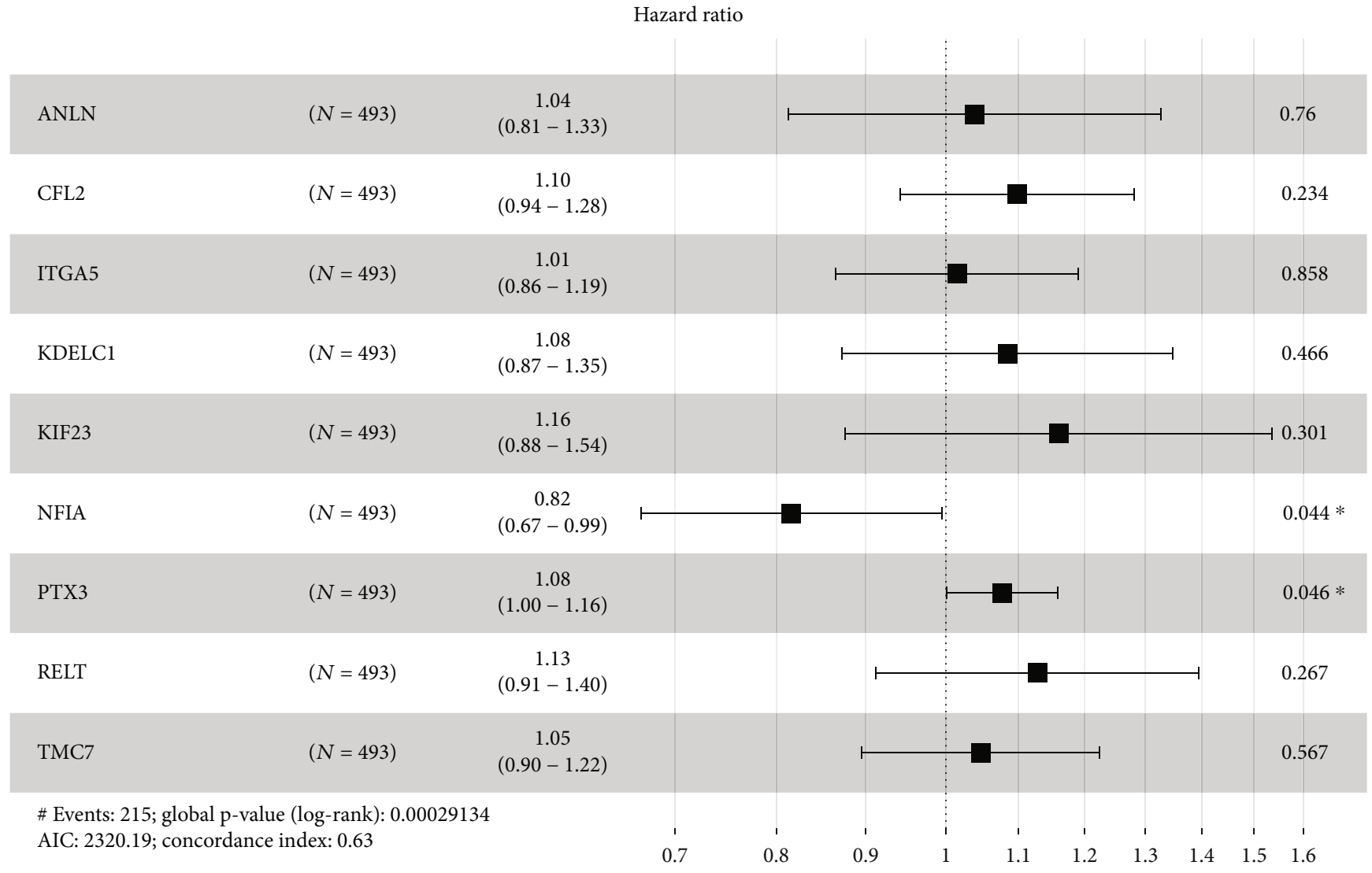

(c)

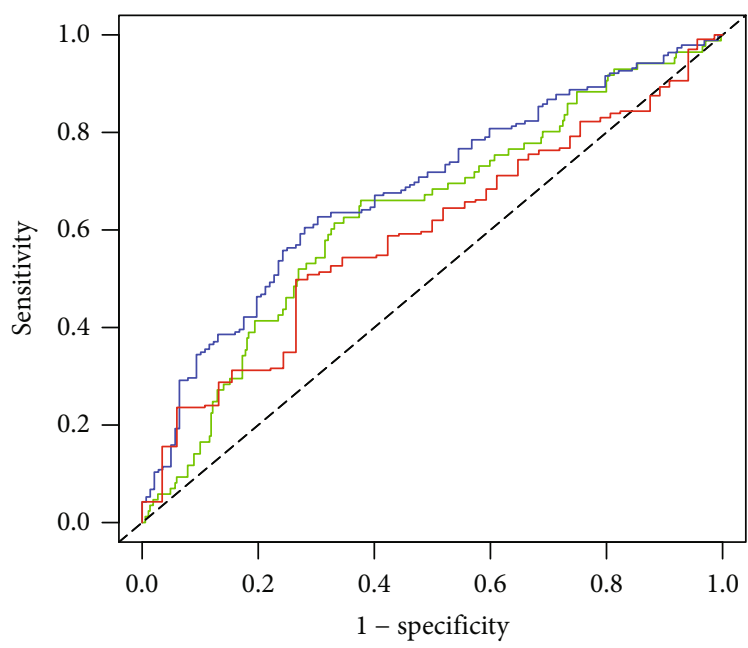

AUC at 1 years: 0.633

AUC at 3 years: 0.681

— AUC at 5 years: 0.591

(d)

Figure 3: Continued. 

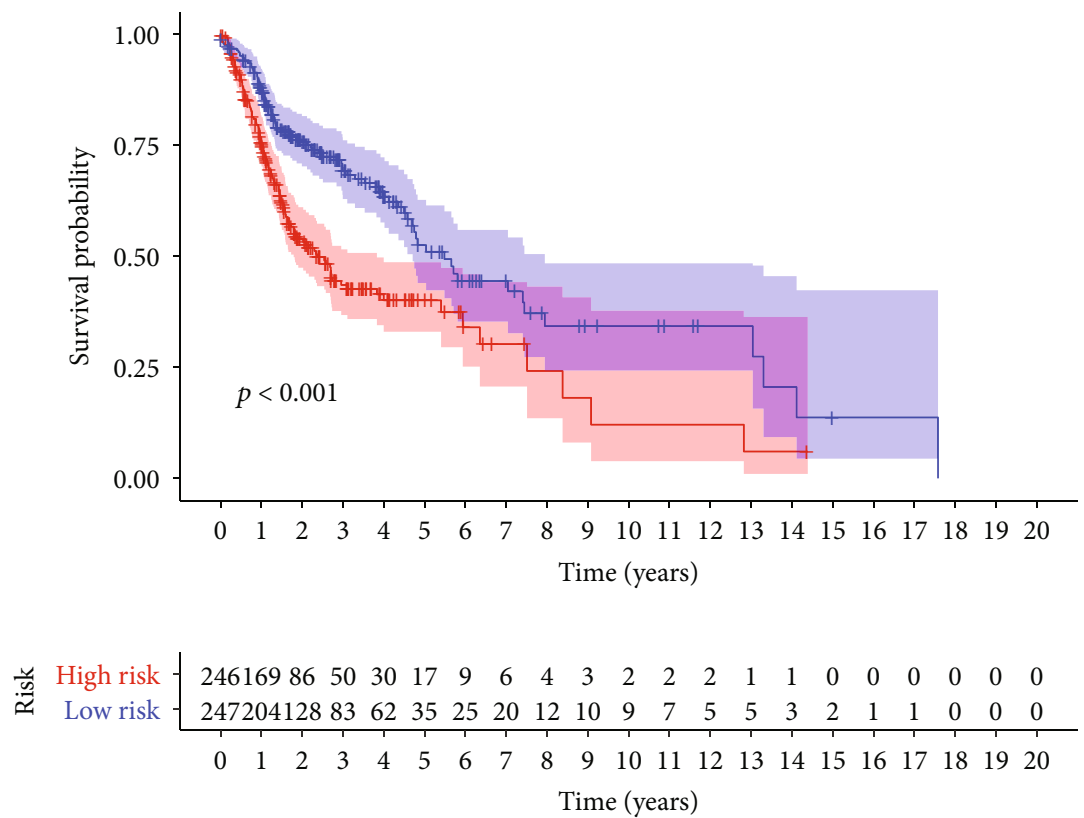

Risk

+ High risk
+ Low risk

(e)

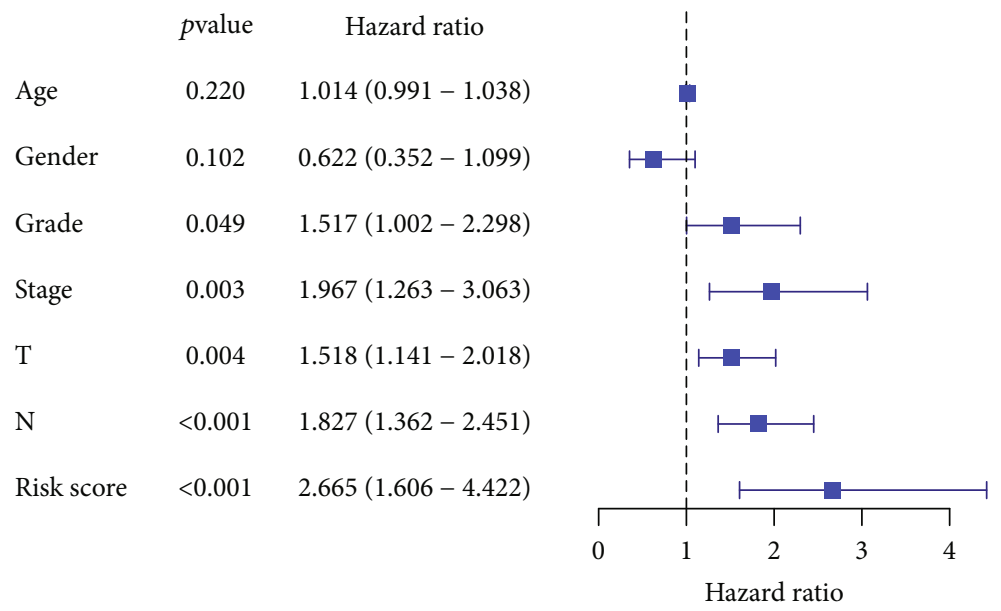

(f)

Figure 3: Continued. 


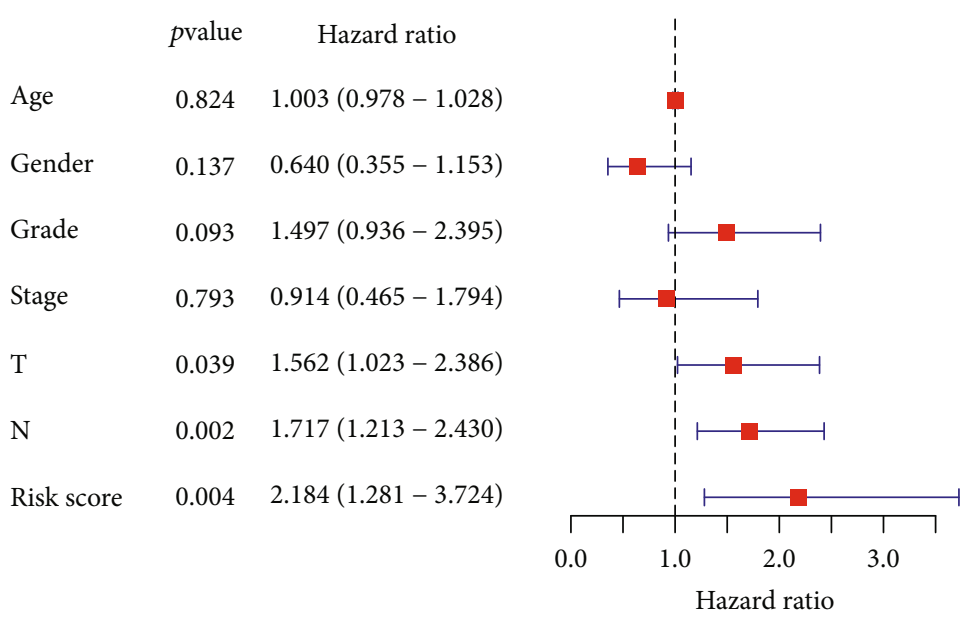

(g)

Figure 3: Development and validation of the ceRNA-related prognostic model. (a-c) LASSO and multivariate Cox regression analyses were applied to investigate the correlation between OS and ceRNAs. (d) ROC analysis showing the accuracy of the prediction model. (e) K-M survival curves comparing the high-risk and low-risk groups and the demonstrating predictive ability of our model. (f, g) Assessment of the independence of ceRNA-related prognostic model through (f) univariate and (g) multivariate Cox regression analyses. HR $>1$ and $P$ value $<0.05$ indicate poor prognostic factors. $\mathrm{HR}<1$ and $P$ values $<0.05$ indicate favorable prognostic factors.

multivariable model analysis. Only two key biomarkers (NFIA and PTX3) had a significant impact on HNSCC prognosis (Figure 3(c)). In addition, analysis of the ROC curves indicated that the AUCs for 1-, 3-, and 5-year OS were $0.633,0.681$, and 0.591 , respectively (Figure $3(\mathrm{~d})$ ). We further plotted survival curves for the high- and low-risk groups. As shown in Figure 3(e), patients in the low-risk group had significantly longer OS probability than those in the high-risk group. Finally, univariate and multivariate Cox regression analyses demonstrated that the risk score based on key ceRNAs was an independent predictor of poor prognosis in HNSCC patients (Figures 3(f) and 3(g)).

3.4. Immune Cell Infiltration Analysis. CIBERSORT was used to assess immune cell infiltration in each HNSCC sample. The results revealed significant differences in the proportions of immune cell infiltration (Figure 4(a)). In addition, the generated heatmap showed that 11 immune cell subtypes, M1 macrophages, CD8 T cells, M0 macrophages, M2 macrophages, resting CD4 memory T cells, memory $\mathrm{B}$ cell, naïve $\mathrm{B}$ cells, and regulatory $\mathrm{T}$ cells (Treg), monocytes, resting myeloid dendritic cells, and activated mast cells presented significant different proportions between the tumor group and the normal group (Figure 4(b)).

3.5. Construction and Validation of Immune Cell-Related Prognostic Model. Univariate Cox regression, LASSO regression, multivariate Cox regression, and independent prognostic analysis were used to analyze the associations between different immune cell subtypes and prognosis. The results showed that three variables were related to prognosis in HNSCC: high infiltration of Tregs and naïve $B$ cells was associated with a favorable prognosis, whereas increased levels of neutrophils were correlated with worse prognosis (Figures 4(c)-4(e)). According to ROC curve analysis, the
AUCs of the 1-, 3-, and 5-year prognosis models were $0.625,0.626$, and 0.568 , respectively (Figure $4(\mathrm{f})$ ). The K-M curve analysis indicated that the high-risk group had an unfavorable prognosis (Figure $4(\mathrm{~g})$ ). Finally, we found that the immune cell-related risk score was an independent factor for predicting prognosis in HNSCC (Figures 4(h) and 4(i)).

3.6. Relationships between Key ceRNAs and Significant Immune Cell Signatures. There were significant correlations between key molecules in the ceRNA network and immune cell signatures (Figure 5(a)). The coexpression analysis results indicated that CFL2, ITGA5, KDELC1, and TMC7 were negatively associated with levels of naïve $\mathrm{B}$ cell infiltration (Figures 5(b)-5(e)), whereas ANLN, ITGA5, KIF23, and TMC7 expression was negatively associated with Treg levels (Figures 5(f)-5(i)). As shown in Figures 5(j)-5(l), significant immune cell signatures in different risk score exhibited statistical significance, and higher levels of naïve B cells were associated with lower risk scores $(P=0.0085)$. A similar result was observed for Tregs $(P=4 e-9)$.

3.7. Clinical Correlation Analysis. We evaluated the correlations of the nine key signatures with tumor grade and stage. Our results showed that increased expression of ANLN and KIF23 was associated with higher tumor grade (Figures 6(a) and 6(b)). Moreover, the expression levels of ITGA5, KDELC1, and PTX3 were positively correlated with the stage of HNSCC, whereas NFIA expression decreased at higher HNSCC stages (Figures 6(c)-6(f)). Furthermore, our results showed that ANLN, KDELC1, KIF23, and NFIA expression was associated with different immune subtypes. ANLN and KIF23 showed increased expression in subtypes $\mathrm{C} 1$ and C2. KDELC1 showed higher expression in the C1, C2, and C6 subtypes, indicating that it may be mainly associated 


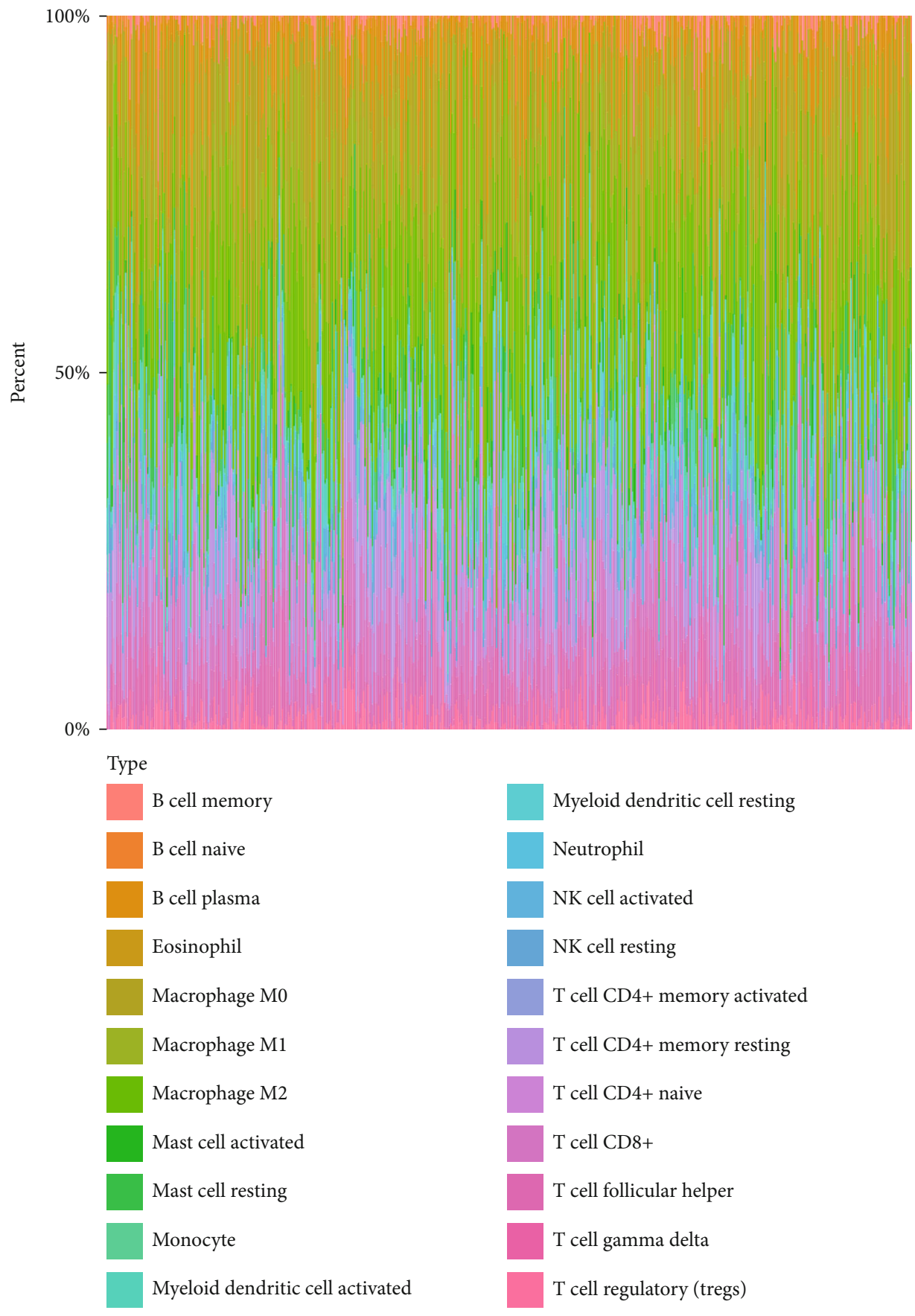

(a)

Figure 4: Continued. 

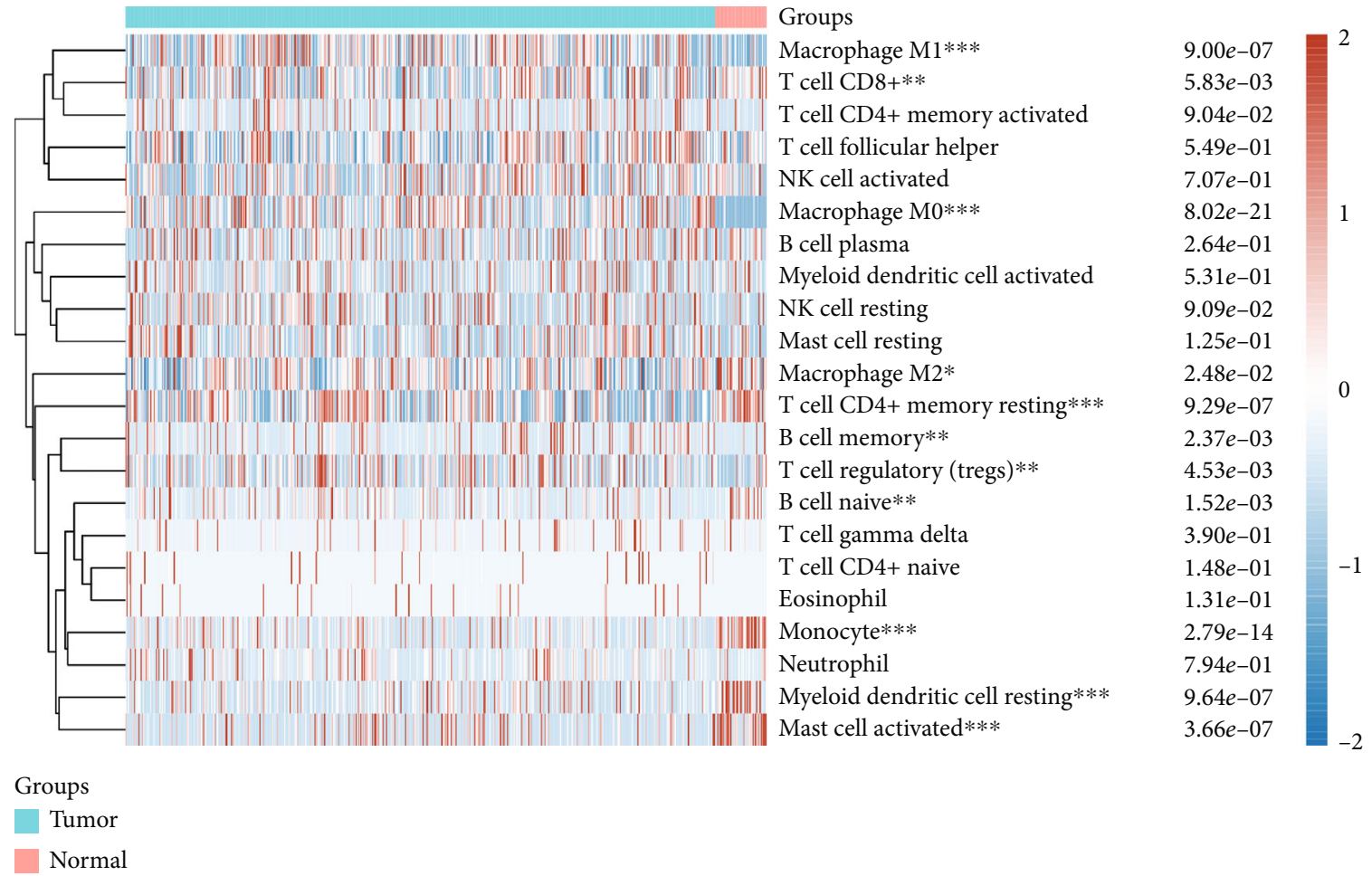

(b)

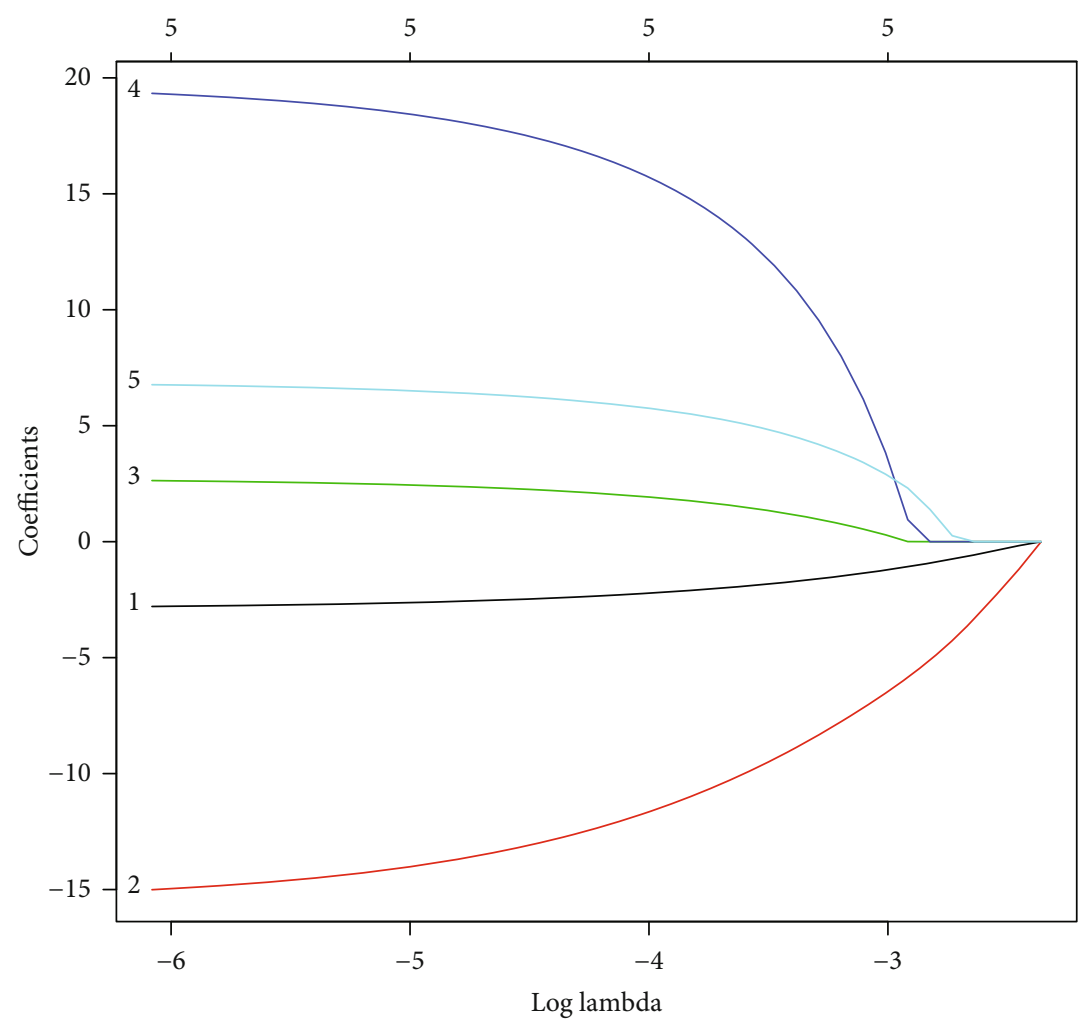

(c)

FIgURE 4: Continued. 


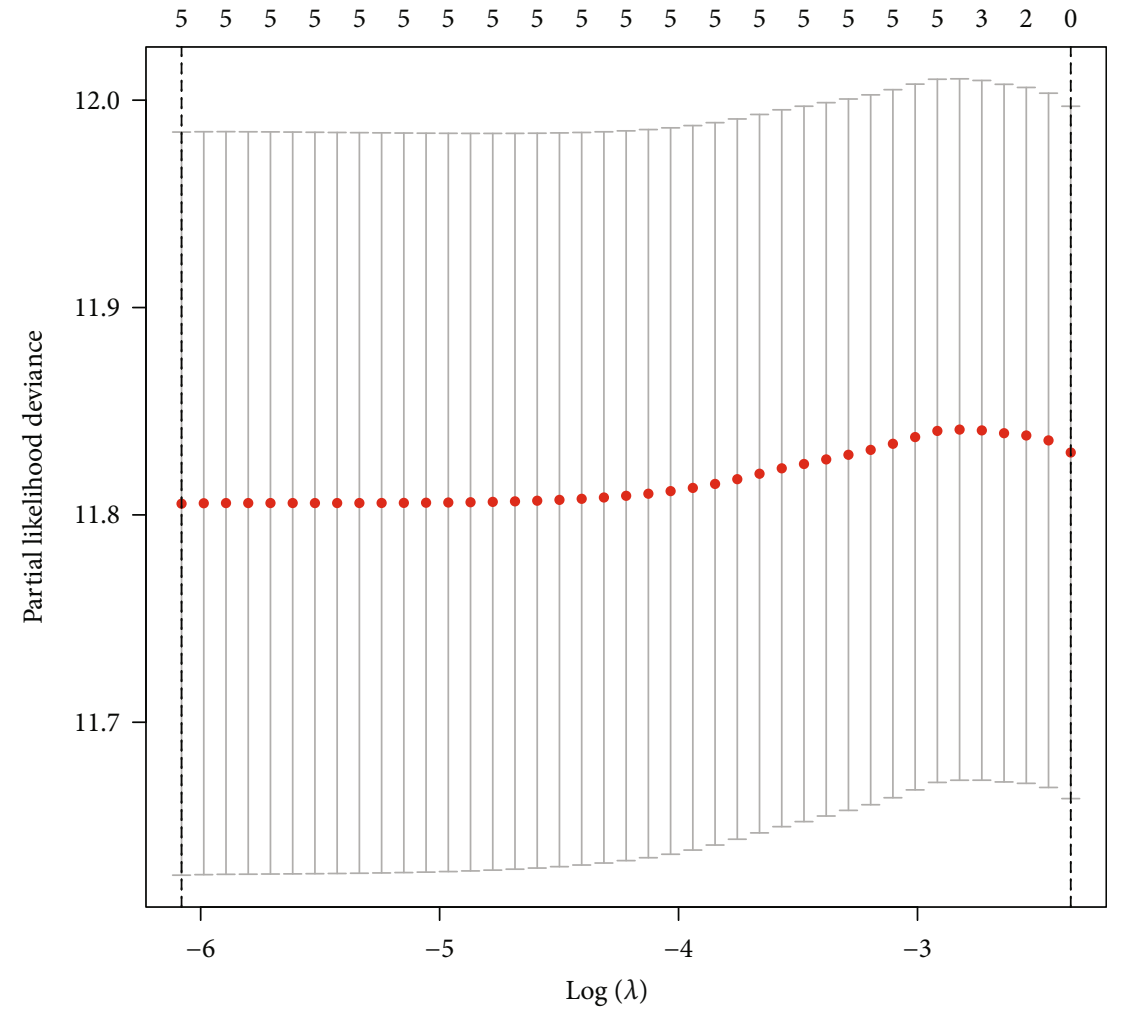

(d)

Hazard ratio

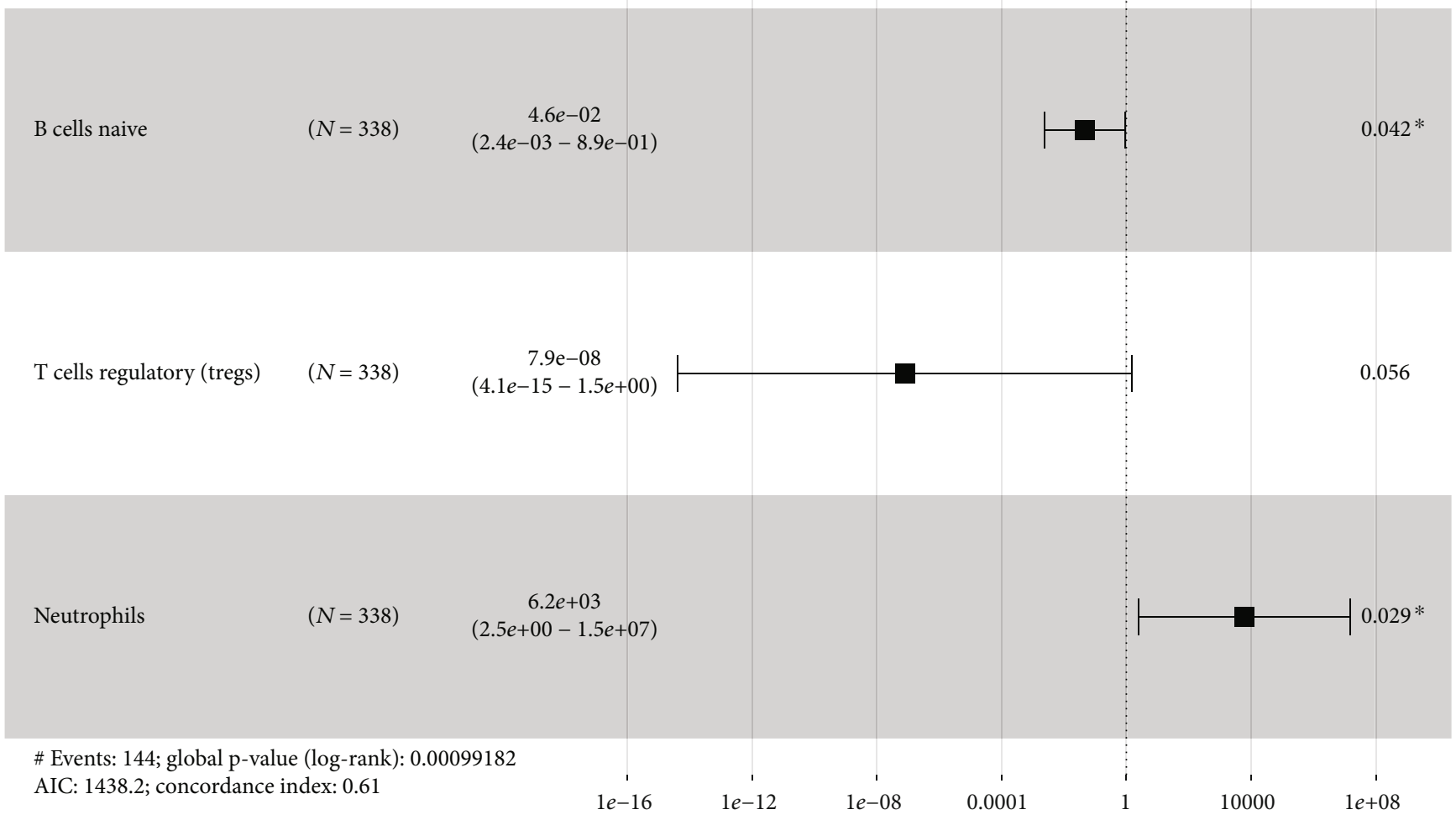

(e)

FIgURE 4: Continued. 


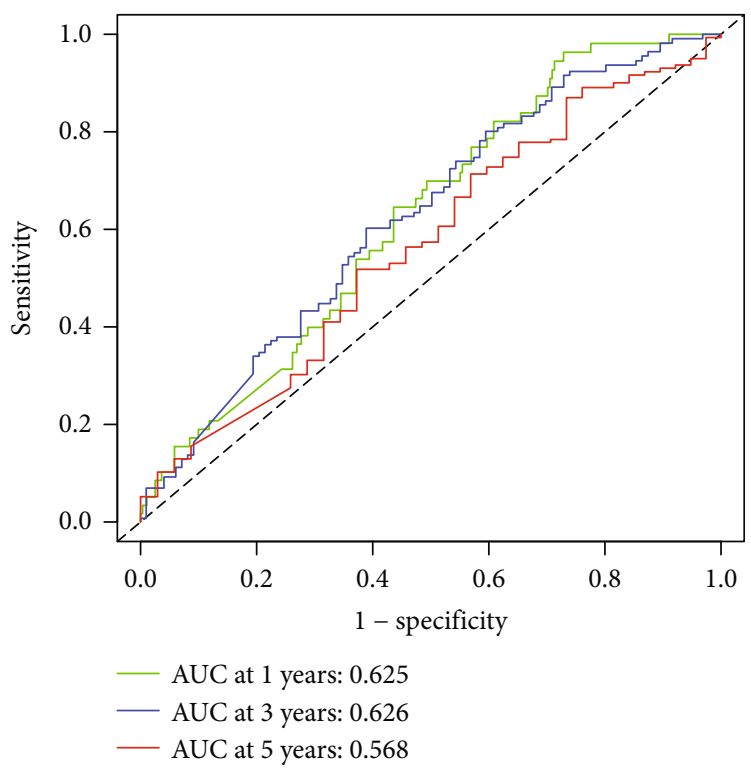

(f)

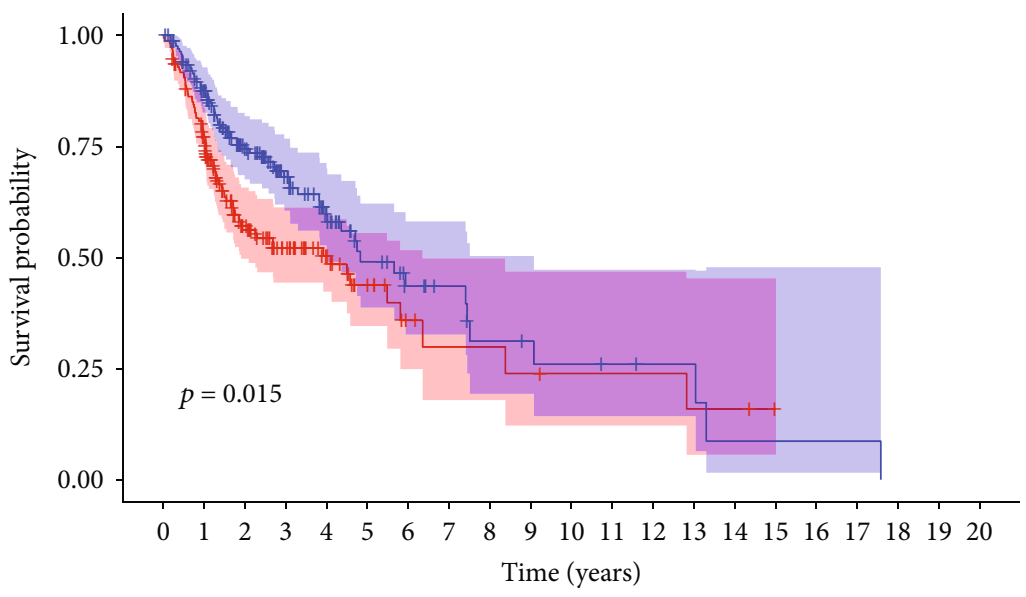

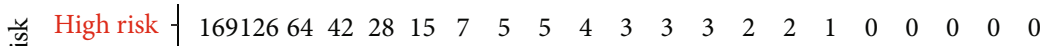

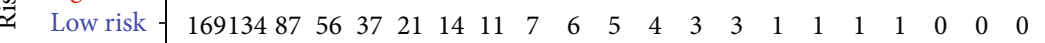

$\begin{array}{lllllllllllllllllllll}0 & 1 & 2 & 3 & 4 & 5 & 6 & 7 & 8 & 9 & 10 & 11 & 12 & 13 & 14 & 15 & 16 & 17 & 18 & 19 & 20\end{array}$ Time (years)

Risk

+ High risk

+ Low risk

(g)

FIgUre 4: Continued. 


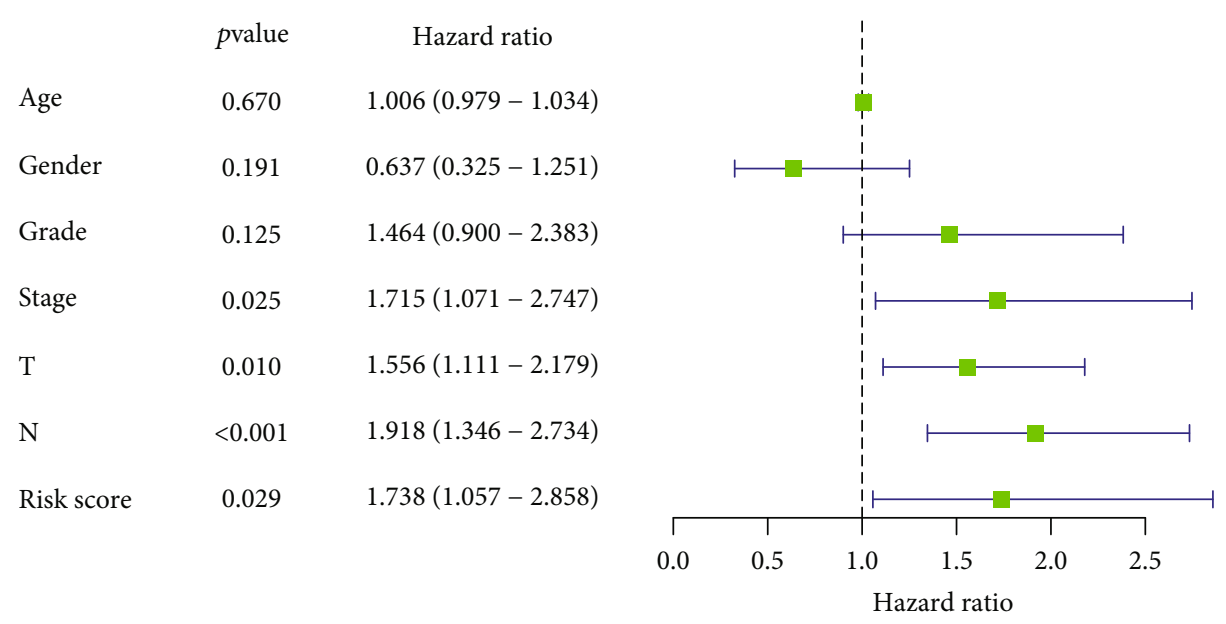

(h)

$\begin{array}{lcc} & p \text { value } & \text { Hazard ratio } \\ \text { Age } & 0.762 & 0.996(0.968-1.024) \\ \text { Gender } & 0.102 & 0.562(0.281-1.122) \\ \text { Grade } & 0.011 & 2.183(1.194-3.992) \\ \text { Stage } & 0.054 & 0.466(0.215-1.012) \\ \mathrm{T} & 0.005 & 2.171(1.261-3.735) \\ \mathrm{N} & <0.001 & 2.543(1.631-3.964) \\ \text { Risk score } & 0.006 & 2.398(1.281-4.488)\end{array}$

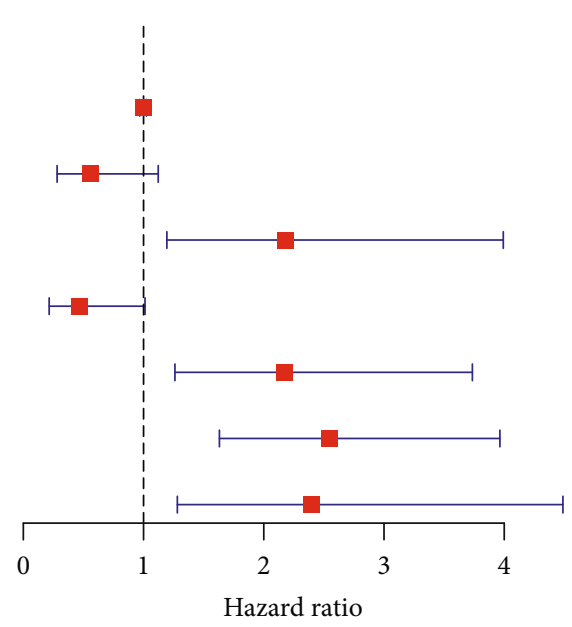

(i)

FIGURE 4: Construction and validation of the immune cells related prognostic model. (a) Distribution of 22 immune cell subtypes estimated by CIBERSORT in HNSCC. (b) Heatmap showing the levels of 22 immune cell subtypes in tumor and normal samples. (c-e) LASSO and multivariate Cox regression were used to investigate the correlation between OS and immune cell infiltration. (f, g) ROC and Kaplan-Meier survival curves showing the accuracy of immune cell subtypes and related prognostic models for predicting OS. (h) Univariate analysis and (i) multivariate Cox regression analysis confirmed the independence of the immune cell-related prognostic model.

with worse survival. NFIA showed higher expression in subtype C3 and decreased expression in C4, which predicted better survival (Figures 6(g)-6(i)). CFL2, ITGA5, KDELC1, and PTX3 were highly expressed in the MS subtype. Increased expression of RLET and TMC7 was mainly found in the basal subtype, and higher expression of NFIA was associated with the AT subtype (Figures 6(k)-6(q)).

3.8. Key ceRNA Expression Was Correlated with Immunotherapy Response and Drug Sensitivity. Multiple IC genes including CD274, CTLA4, HAVCR2, LAG3, PDCD1, SIGLEC15, TIGIT, and TIM3 exhibited higher expression in tumor tissues compared with normal tissues (Figure 7(a)). The expression of ANLN, ITGA5, and KIF23 was positively associated with the expression of CD274 (PDL1), HAVCR2, and SIGLEC15. CFL2 and RELT expression was positively correlated with the expression of CD274 (PDL1), CTLA4, HAVCR2, LAG3, PDCD1 (PD1), SIGLEC15, and TIGIT.
NFIA was positively related to the abovementioned seven immunosuppressive genes, except for CD274. Overexpression of PTX3 was associated with increased CTLA4, HAVCR2, SIGLEC15, and TIGIT expression. TMC7 expression was negatively correlated with the expression of CTLA4, HAVCR2, LAG3, PDCD1 (PD1), and TIGIT (Figure 7(b)).

In addition, our biomarker could effectively predict antiPD1 response compared with published biomarkers (MSI, CD274, CD8, IFNG, etc.) (Figure 7(c)). Moreover, Spearman's correlation analysis was performed to explore the correlations of the expression of the nine key genes with drug sensitivity in terms of IC50 values. Our results showed that most drugs were effective in association with increased expression of CFL2, TMC7, PTX3, ANLN, NFIA, and KIF23, whereas RELT and ITGA5 were negatively regulated by most drugs. Specifically, these molecules could be exploited as potential therapeutic drug targets for HNSCC (Figure $7(\mathrm{~d})$ ). 


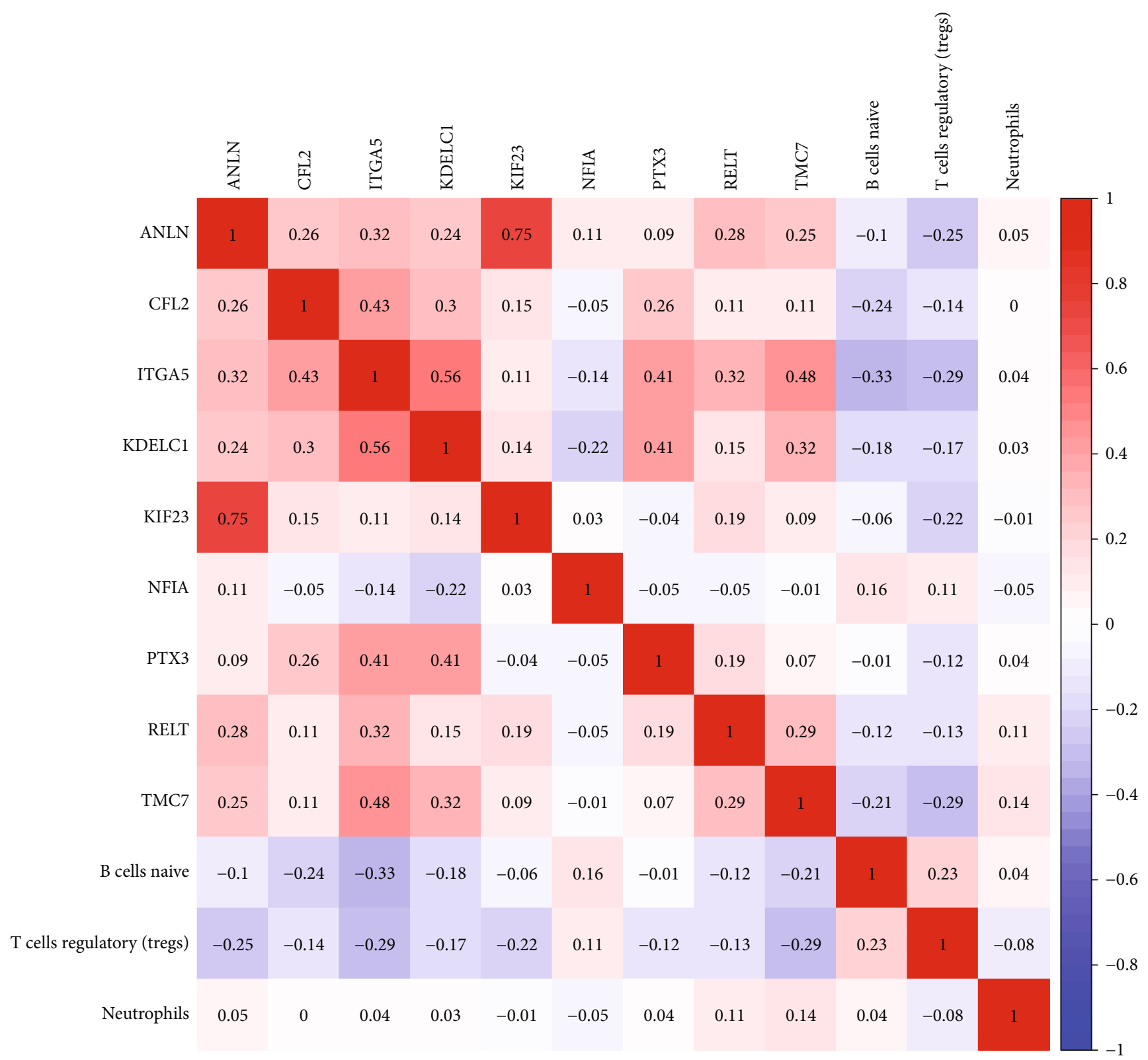

(a)

FIgURE 5: Continued. 


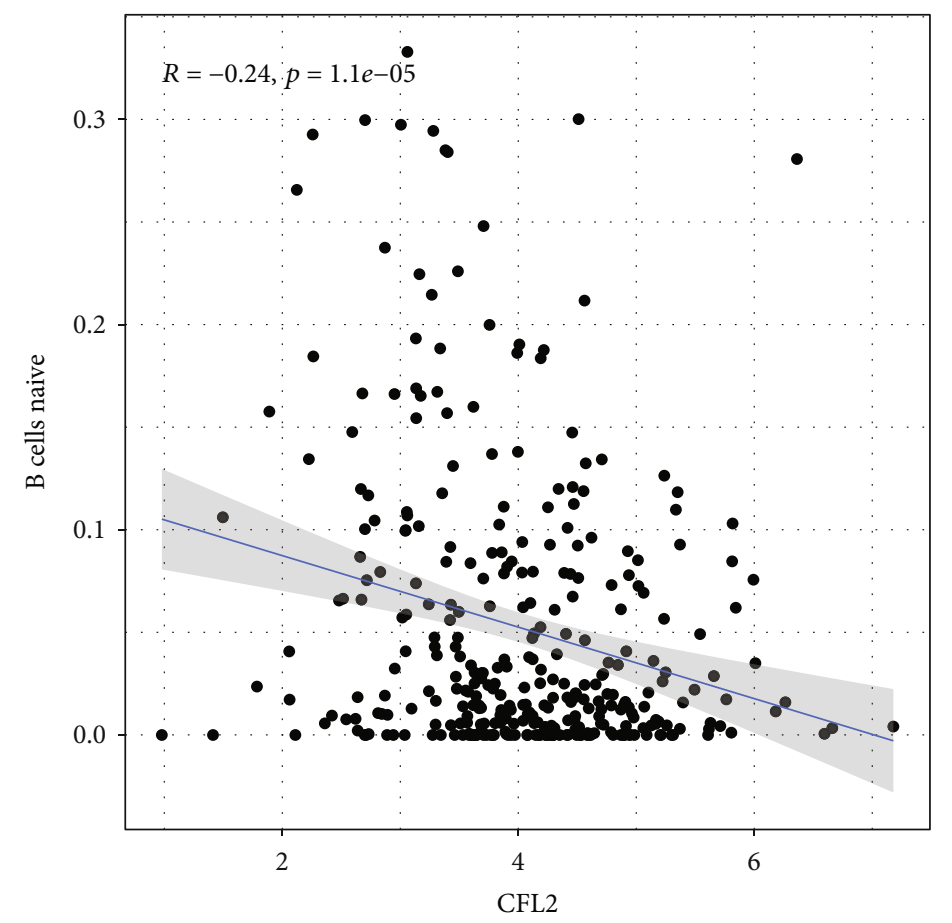

(b)

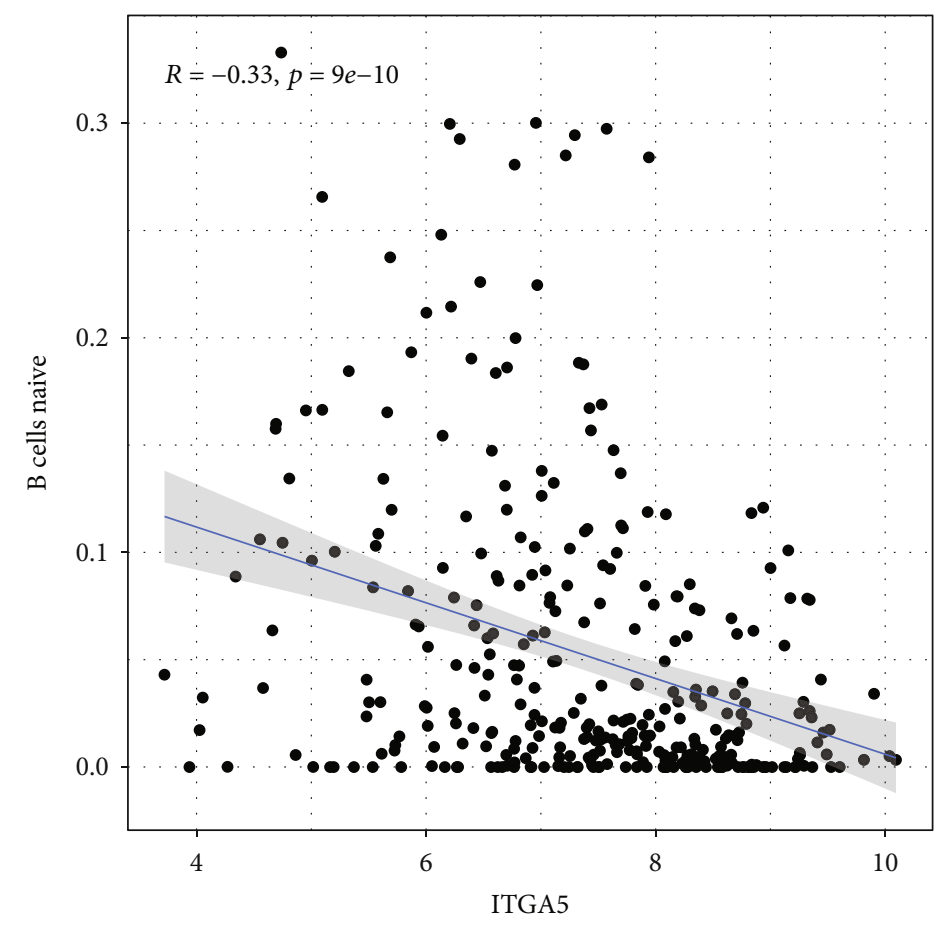

(c)

Figure 5: Continued. 


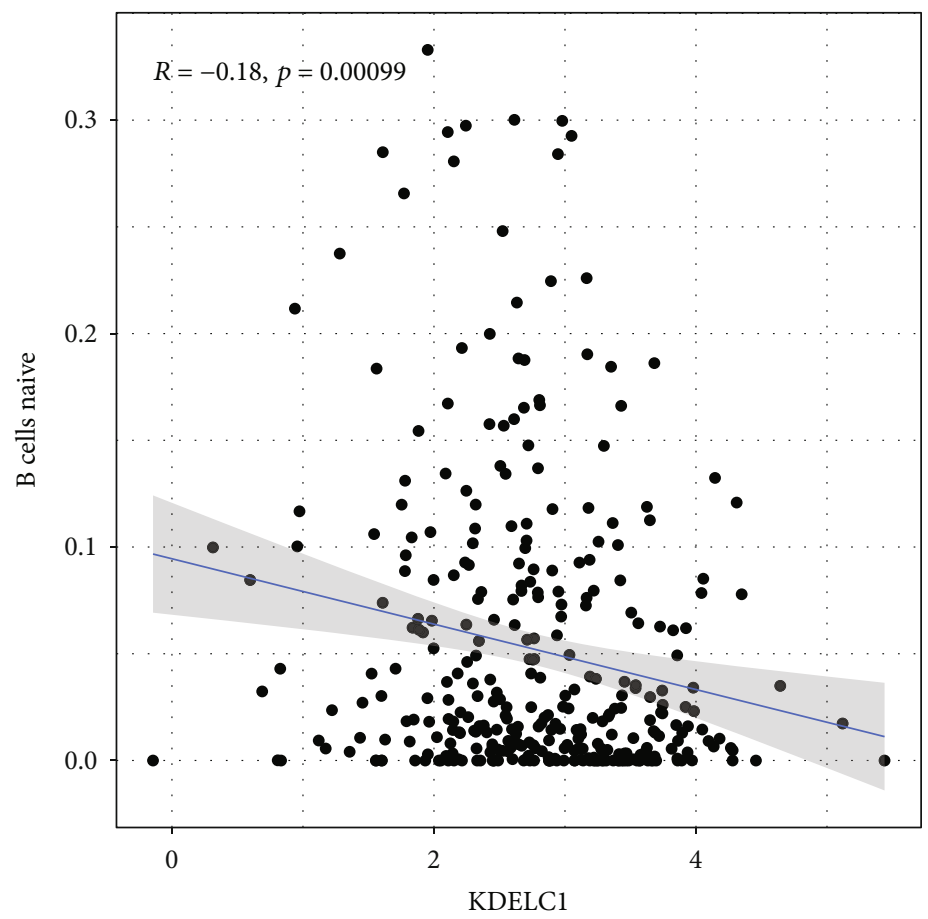

(d)

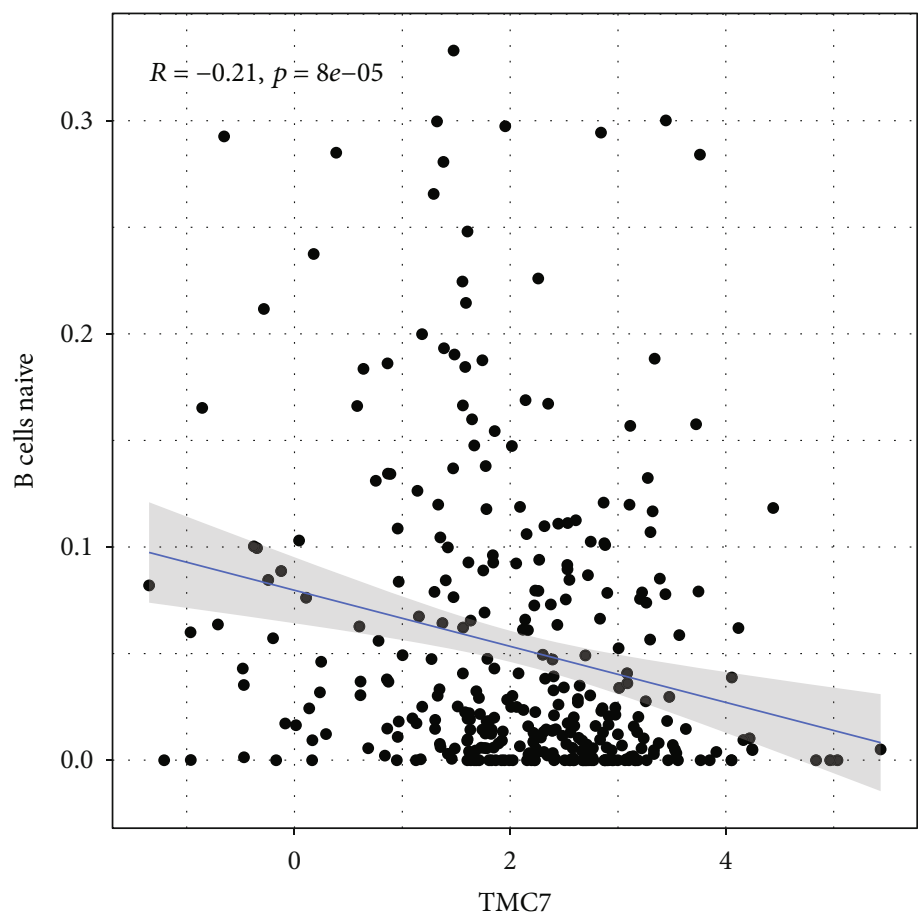

(e)

Figure 5: Continued. 


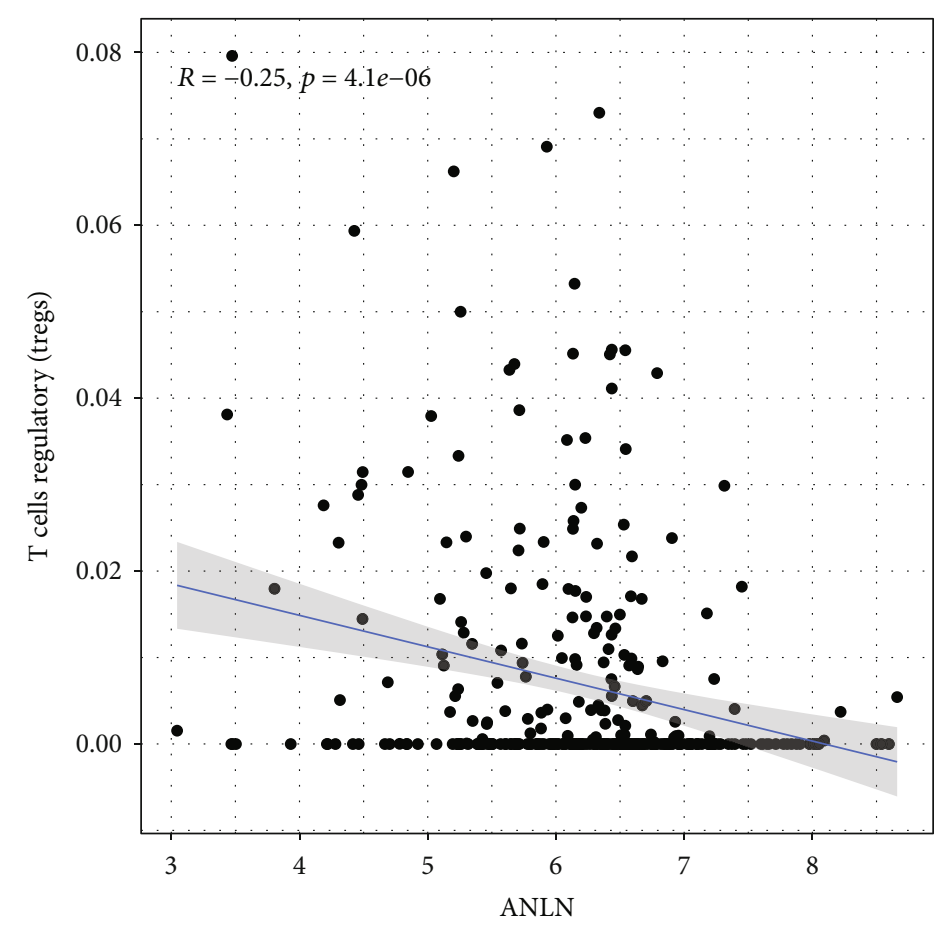

(f)

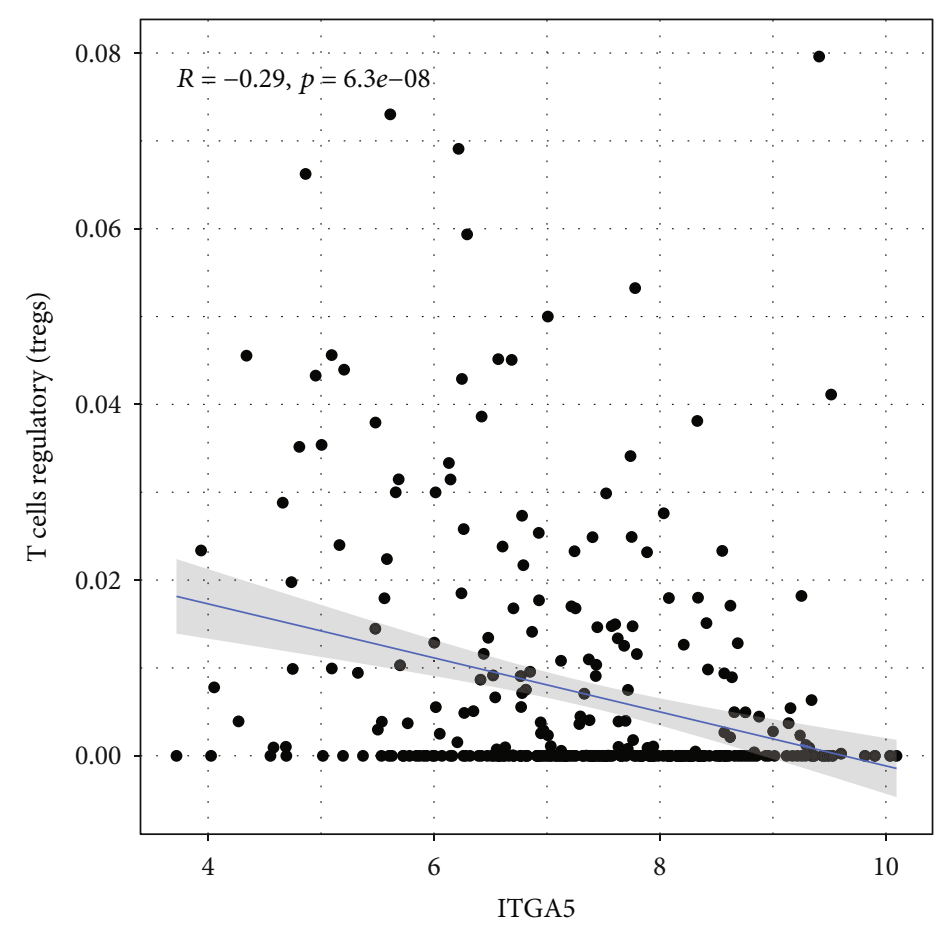

(g)

Figure 5: Continued. 


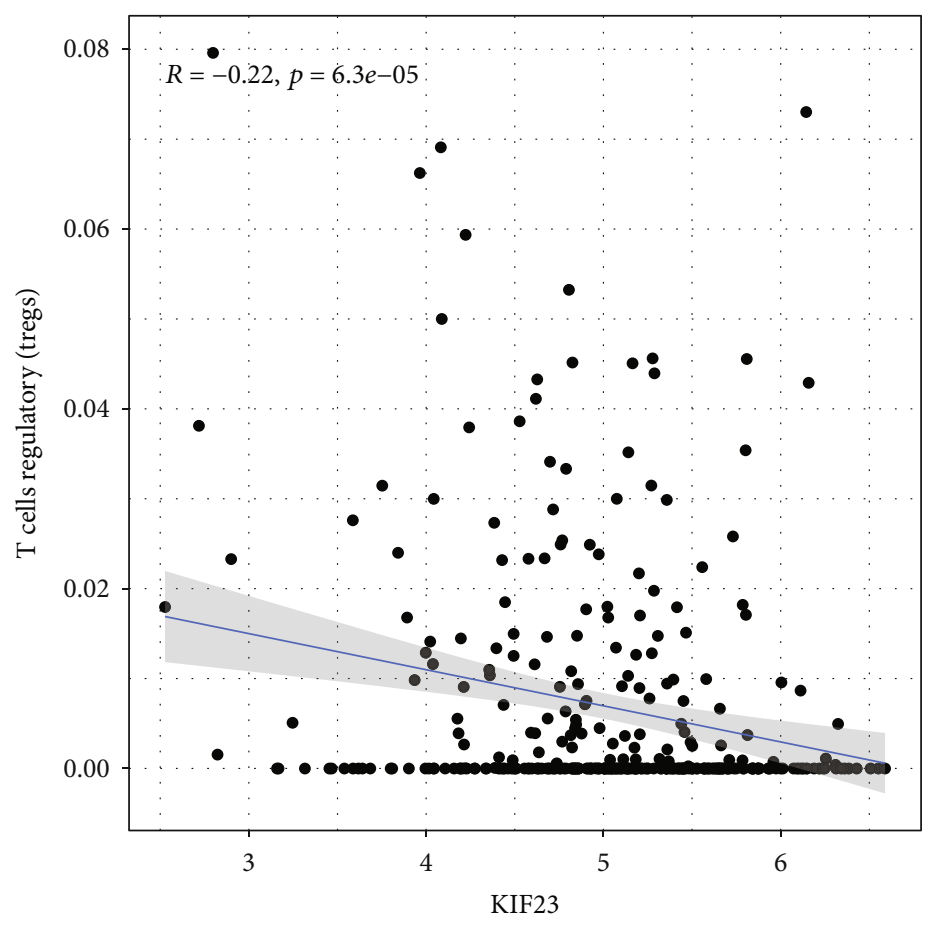

(h)

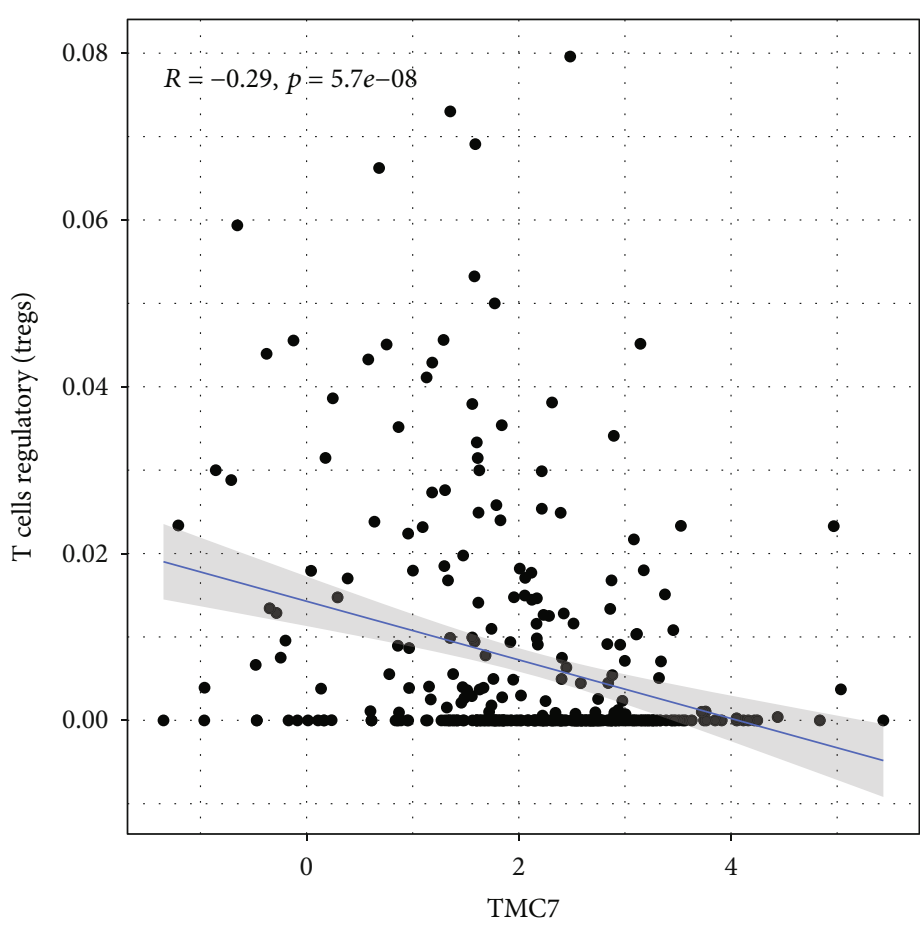

(i)

Figure 5: Continued. 


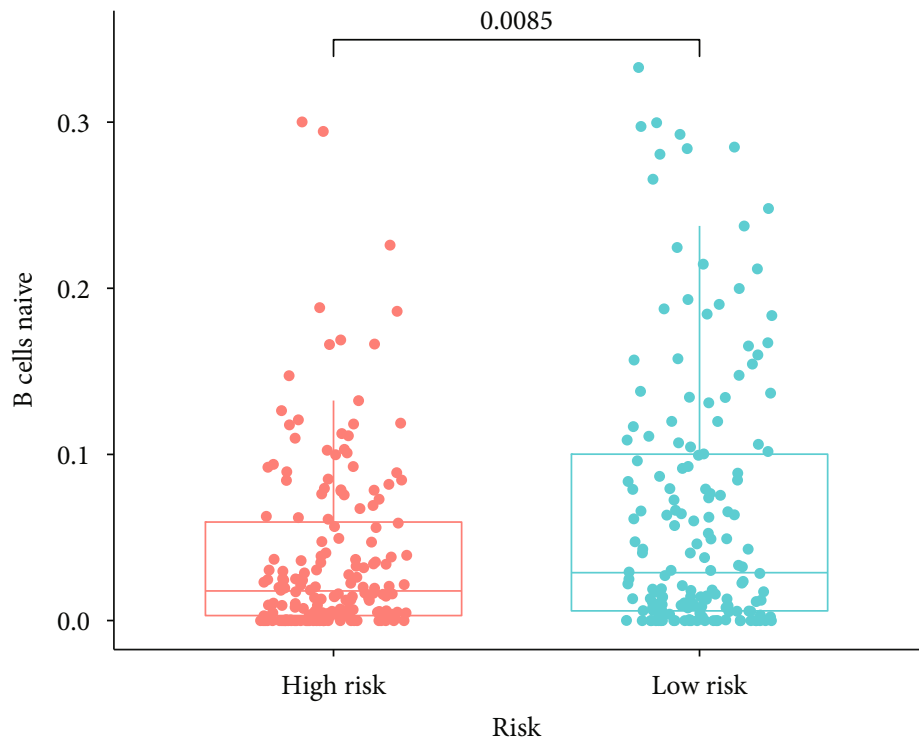

Risk

High risk

Low risk

(j)

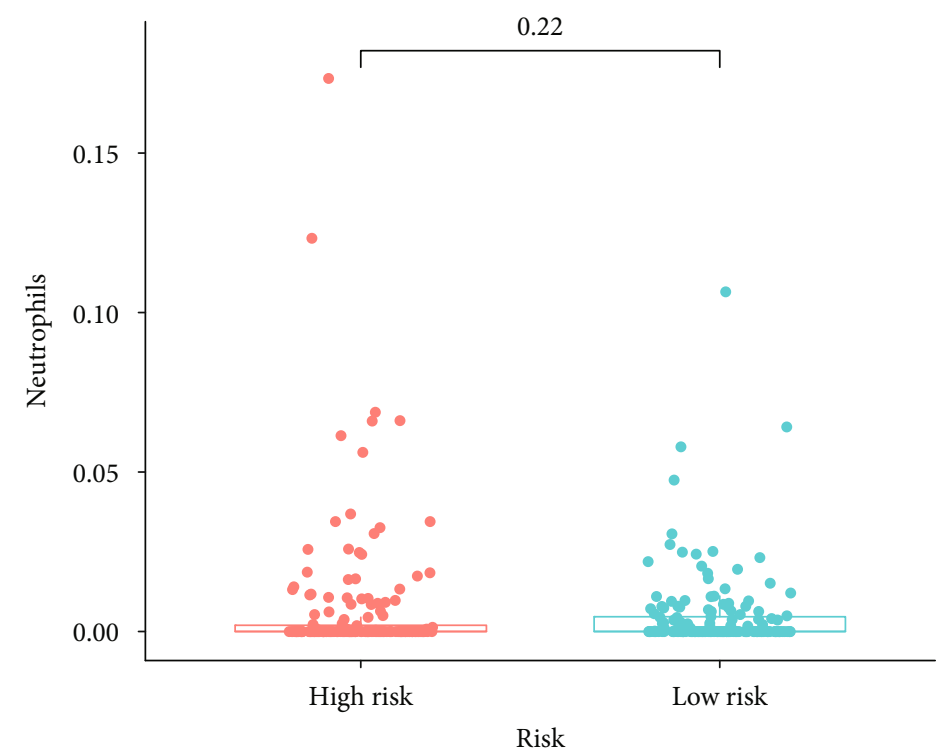

Risk

High risk

Low risk

(k)

Figure 5: Continued. 


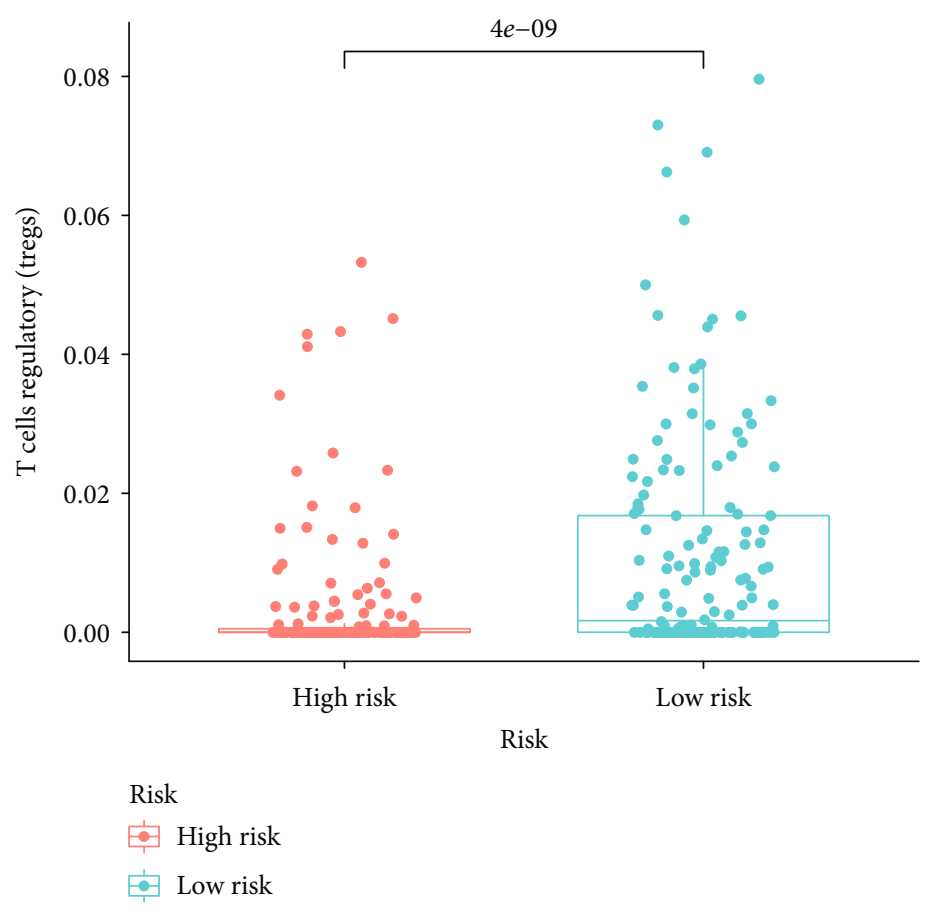

(1)

FIgURE 5: Analysis of the correlations of significant genes in the ceRNA network and gene risk score with immune cell infiltration. (a) Heatmap displaying correlations between prognostic immune cells and key genes in the ceRNA network. (b-e) Expression of CFL2, ITGA5, KDELC1, and TMC7 was negatively associated with infiltration of naïve B cells. (f-i) ANLN, ITGA5, KIF23, and TMC7 expression was negatively associated with Tregs. (j-l) Pearson's correlation analysis was performed to evaluate the associations between key immune cells and gene risk score.

3.9. Validation of the Prognostic Value of Biomarkers and Associations between Key ceRNAs and IC Genes. LOGpc was used to perform survival analysis for key ceRNAs. The results indicated that higher expression levels of CFL2, ITGA5, KDELC1, PTX3, and RELT were correlated with poor prognosis in HNSCC patients, whereas increased expression of NFIA was associated with longer OS. There was no correlation between ANLN, KIF23, or TMC7 expression and prognosis in HNSCC patients (Table 2). The associations between pivotal ceRNAs and prognosis were then determined using TIMER 2.0. We found that high expression levels of ANLN, CFL2, ITGA5, KDELC1, PTX3, RELT, and TMC7 were significantly associated with shorter OS in HNSCC patients, whereas higher expression of NFIA was correlated with longer OS. In HPV-positive HNSCC patients, high expression of ITGA5, KDELC1, and TMC7 was associated with poor prognosis, whereas high NFIA expression was correlated with good prognosis. ANLN, CFL2, KDELC1, PTX3, and RELT were associated with unfavorable prognosis in HPV-negative HNSCC (Figure 8(a)). In addition, the prognostic roles of immune cells in HNSCC patients with different HPV statuses were confirmed using the TIMER 2.0 database. In both HPV-positive and HPVnegative HNSCC patients, high infiltration levels of naïve $\mathrm{B}$ cells and Tregs were associated with a favorable prognosis according to several algorithms, whereas high neutrophil infiltration indicated an unfavorable prognosis (Figures 8(b)-8(d)). Eight tumor-related immunosuppres- sive molecules, CD274, CTLA4, HAVCR2, LAG3, PDCD1 (PD1), PDCD1LG2, SIGLEC15, and TIGIT, had higher expression in HNSCC tumor tissues compared with HNSCC normal tissues.

Notably, most IC molecules showed significantly increased expression in HPV-positive HNSCC patients (Figure 8(e)). We also found that the expression of these ICI factors was significantly positively correlated with the expression of seven key mRNAs (ANLN, CFL2, ITGA5, KIF23, NFIA, PTX3, and RELT), whereas the expression of immunosuppressive molecules was negatively associated with TMC7 expression, especially in HPV-positive HNSCC patients (Figure 8(f)). We further discovered that eight key ceRNAs were correlated with response to ICI in other cancers (melanoma, bladder, kidney, and glioblastoma) (Table 3).

These results are consistent with the results of our study.

\section{Discussion}

HNSCC is among the deadliest malignancies in humans and a significant cause of cancer-related deaths worldwide [35]. Despite advances in the screening, diagnosis, and treatment of HNSCC in recent decades, especially in terms of immunotherapy, the prognosis of HNSCC patients remains very poor [36]. Recent studies have explored the roles of immune cell infiltration and ceRNA networks in HNSCC. However, these studies have failed to accurately identify key molecules 


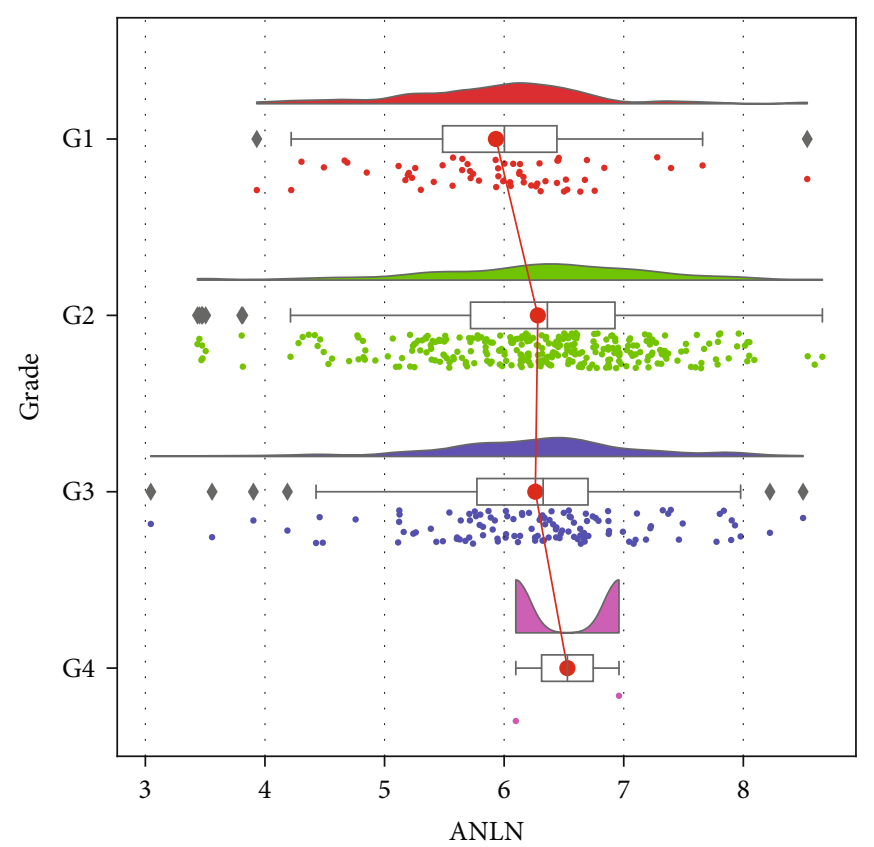

(a)

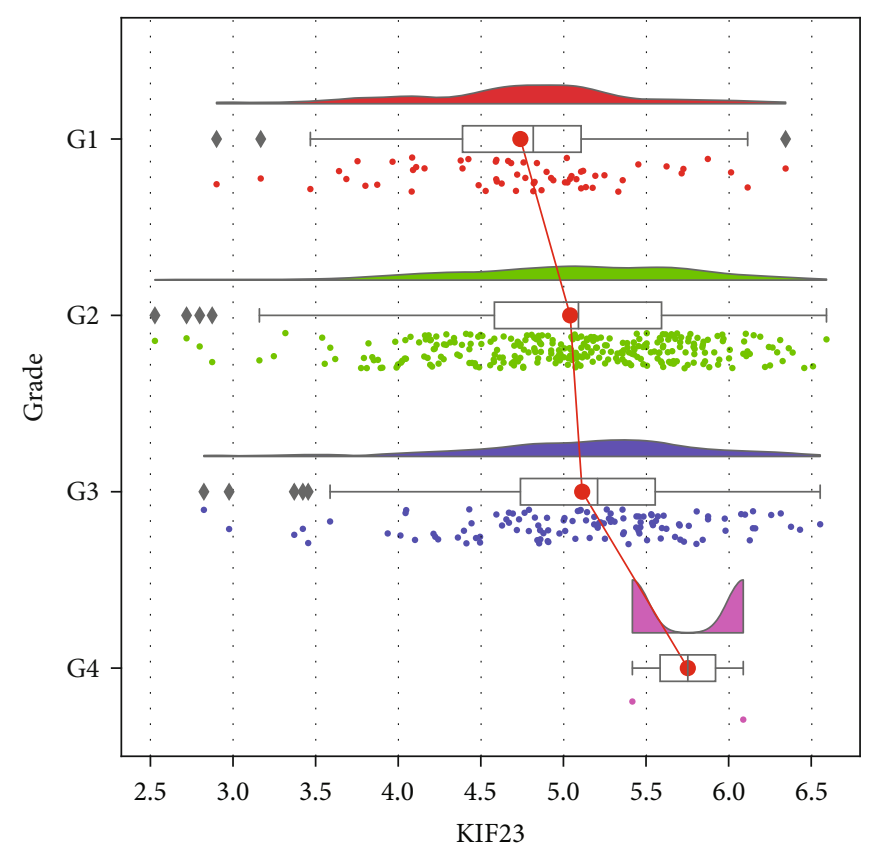

(b)

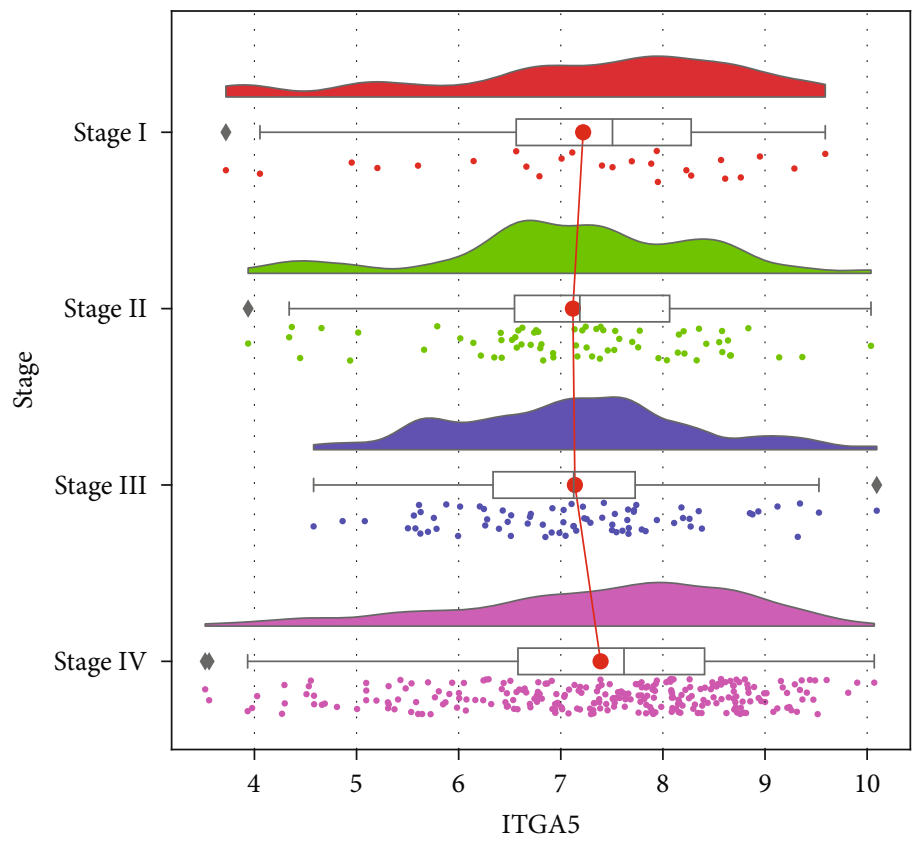

(c)

Figure 6: Continued. 


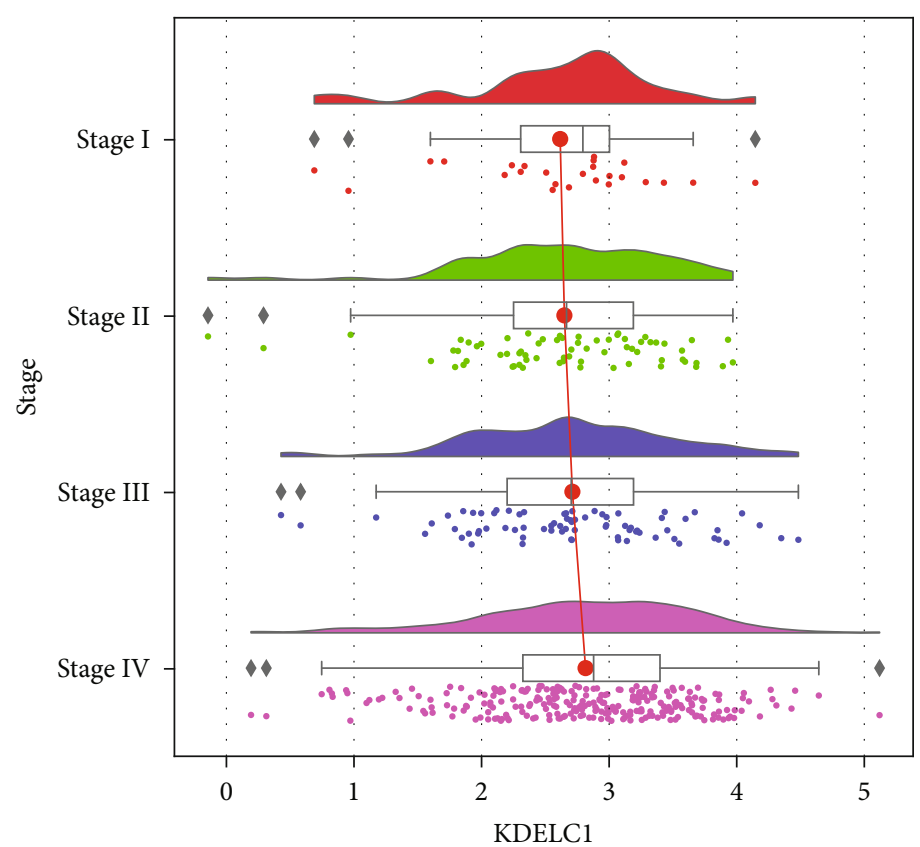

(d)

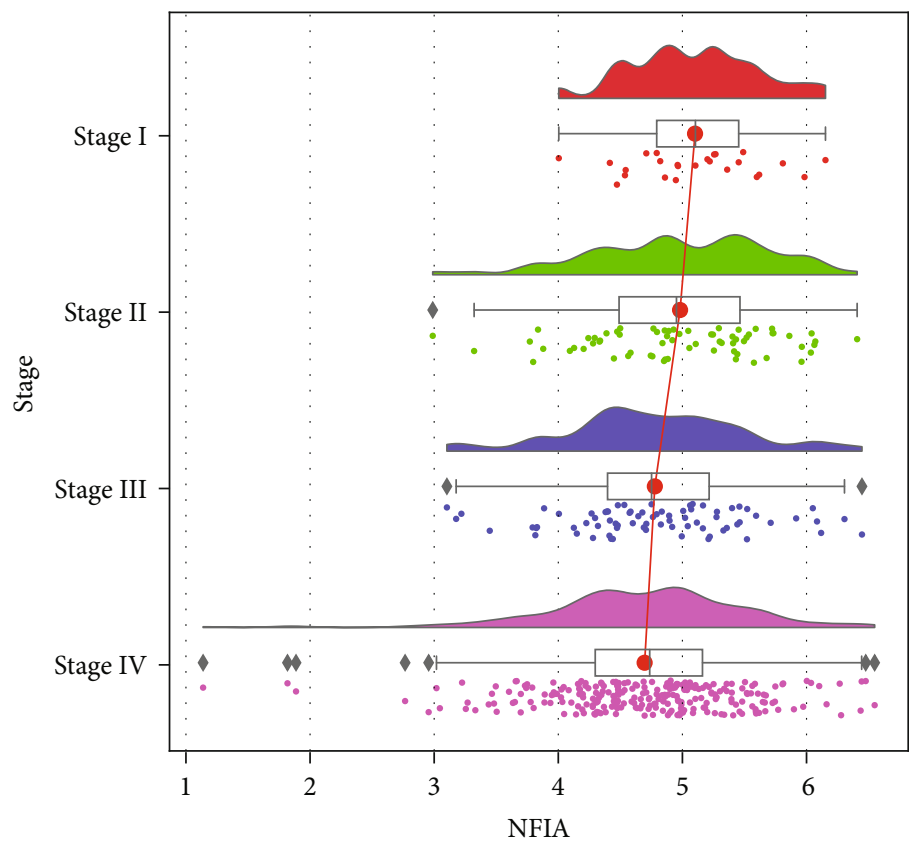

(e)

FIgURe 6: Continued. 


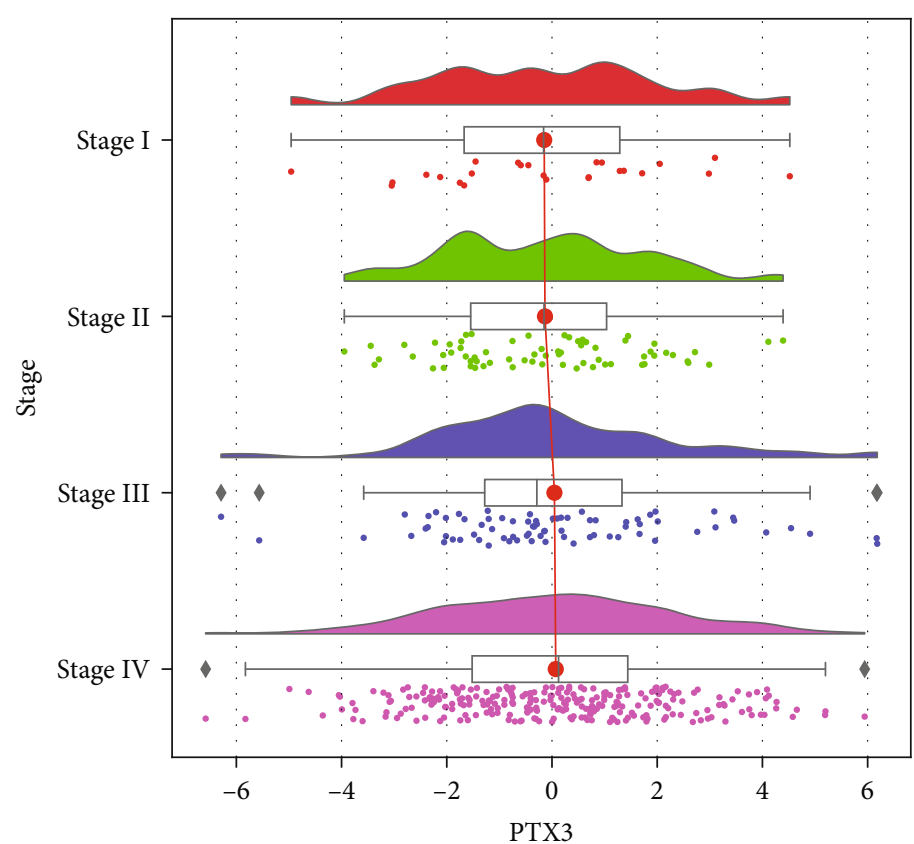

(f)

HNSC :: ANLN_exp

$\mathrm{Pv}=3.51 e-02$

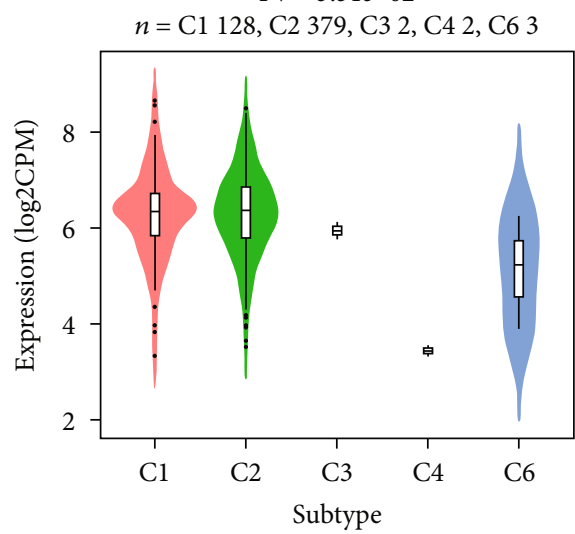

(h)

HNSC :: NFIA_exp

$\mathrm{Pv}=3.78 e-03$

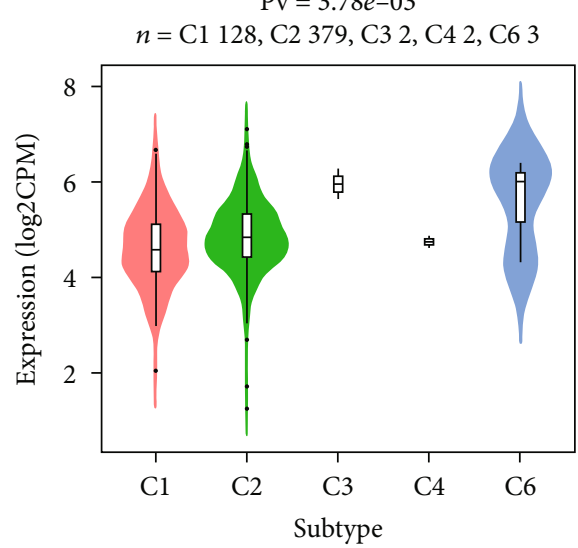

(j)

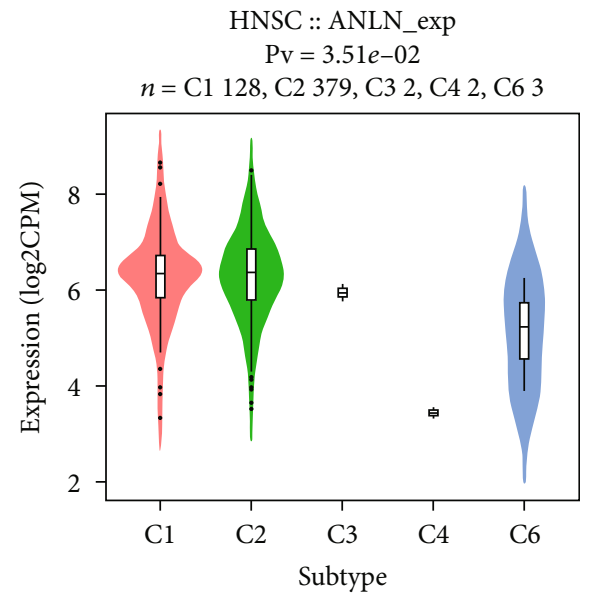

(g)

HNSC :: ANLN_exp

$\mathrm{Pv}=3.51 e-02$

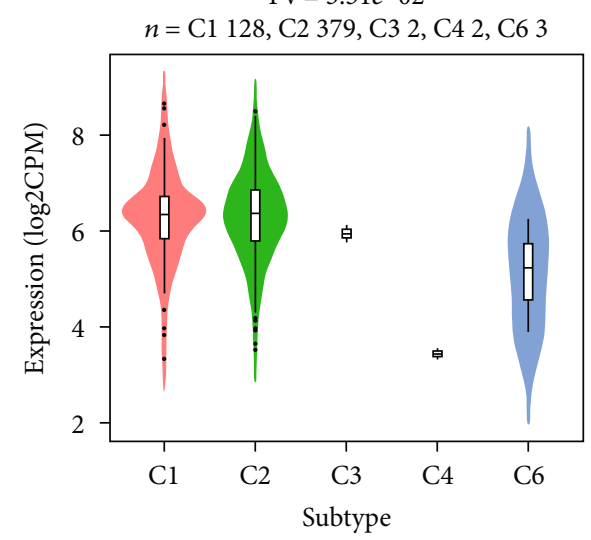

(i)

HNSC :: ANLN_exp

$\mathrm{Pv}=3.51 e-02$

$n=\mathrm{C} 1$ 128, C2 379, C3 2, C4 2, C6 3

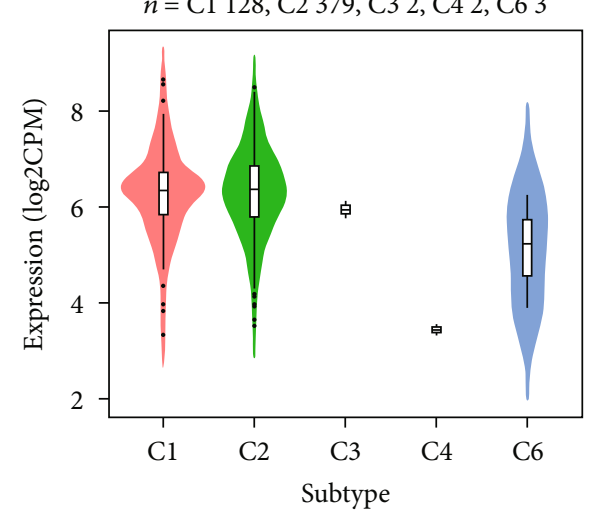

(k)

Figure 6: Continued. 


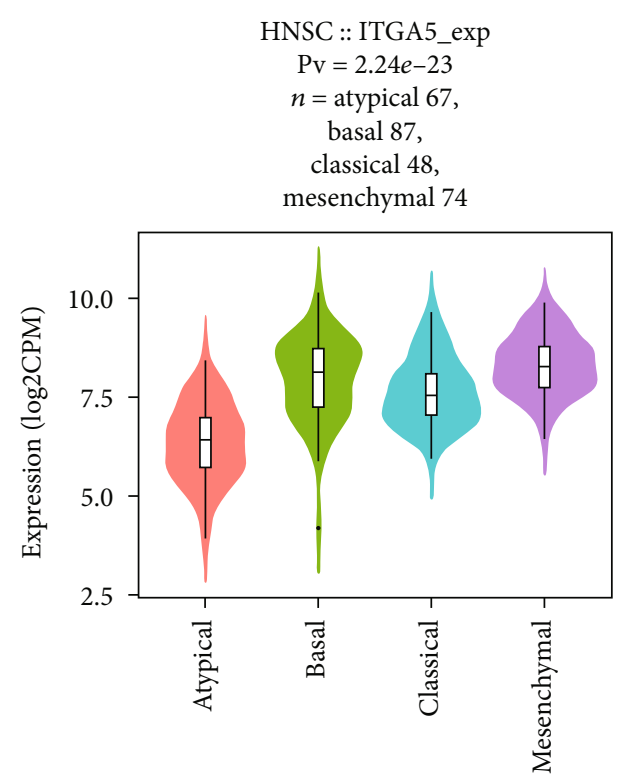

Subtype

(l)

HNSC :: NFIA_exp

$\mathrm{Pv}=3.43 e-07$

$n=$ atypical 67,

basal 87,

classical 48 ,

mesenchymal 74

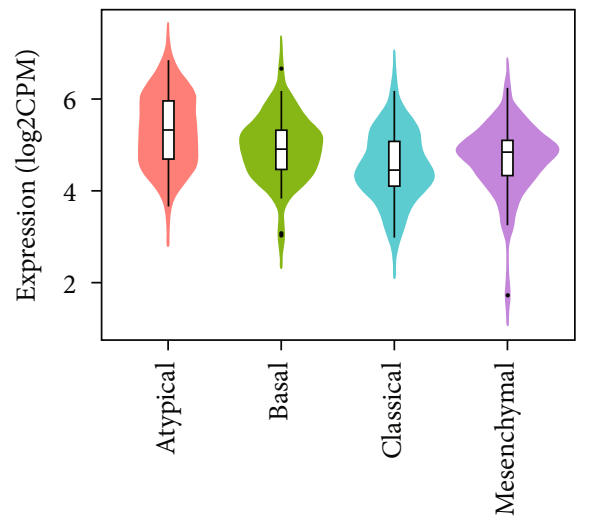

Subtype

(n)
HNSC :: ITGA5 exp

$\mathrm{Pv}=2.24 e-23$

$n=$ atypical 67 ,

basal 87 ,

classical 48

mesenchymal 74

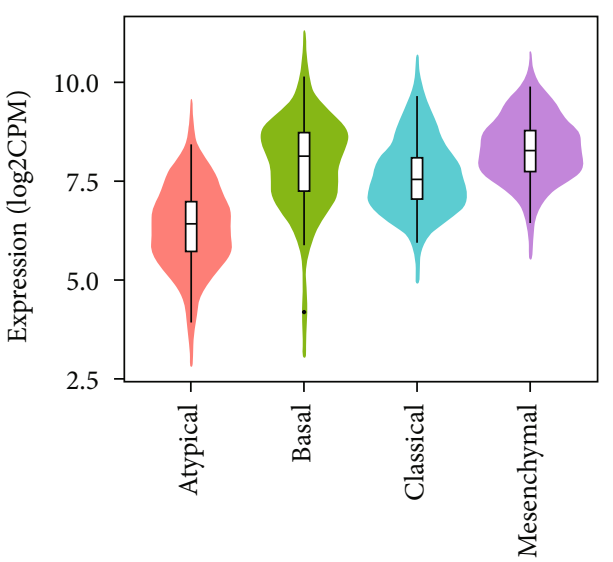

Subtype

(m)

HNSC :: PTX3_exp

$\mathrm{Pv}=2.06 e-11$

$n=$ atypical 67 ,

basal 87 ,

classical 48

mesenchymal 74

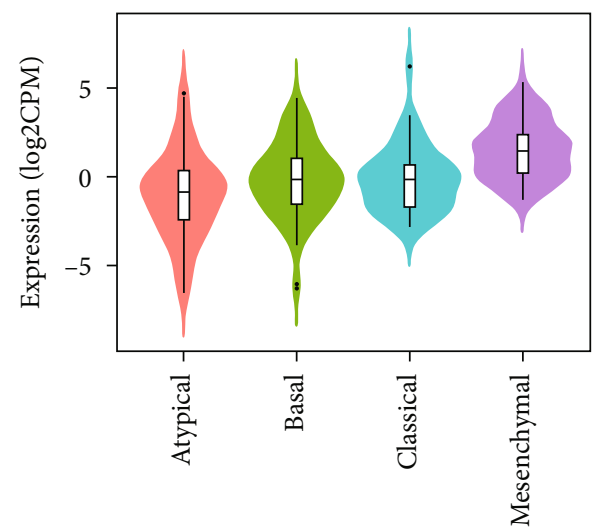

Subtype

(o)

Figure 6: Continued. 


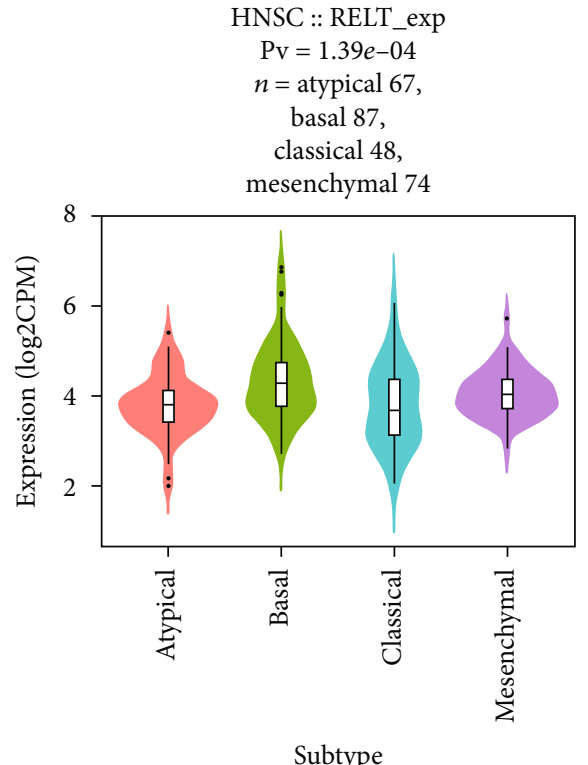

(p)
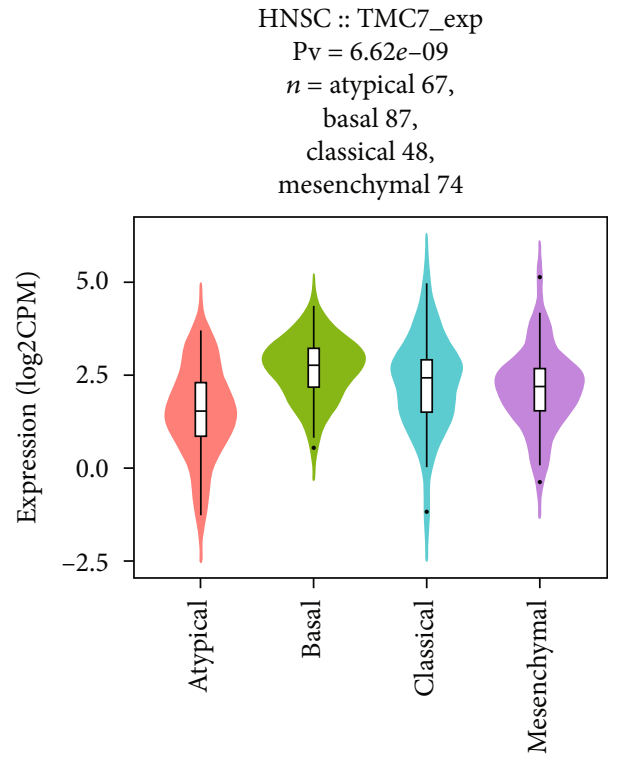

Subtype

(q)

FIGURE 6: Clinical correlation analysis. (a, b) ANLN and KIF23 expression was associated with tumor grade according to TISIDB datasets. (a) ANLN and (b) KIF23. (c-f) Key mRNAs among ceRNAs related to tumor stage according to TISIDB. (c) ITGA5, (d) KDELC1, (e) NFIA, and (f) PTX3. (g-i) Distribution of the expression of key ceRNAs across immune subtypes according to TISIDB: (g) ANLN, (h) KDELC1, (i) KIF23, and (j) NFIA. (k-q) Distribution of key ceRNA expression across molecular subtypes (TISIDB): (k) CFL2, (l) ITGA5, (m) KDELC1, (n) NFIA, (o) PTX3, (p) RELT, and (q) TMC7. Statistical significance of differential expression evaluated using Kruskal-Wallis test. C1 (wound healing), C2 (IFN-gamma dominant), C3 (inflammatory), C4 (lymphocyte depleted), and C6 (TGF- $\beta$ dominant). Molecular subtypes include four types, namely, atypical (AT), basal (BA), classical (CL), and mesenchymal (MS) based on biological characteristics of genes highly expressed in each subtype.

for predicting the prognosis of HNSCC [9, 10,37]. Accumulating evidence suggests that machine learning is a reliable and robust technique that can be used to quickly and accurately identify critical biomarkers [38, 39]. ICIs may exert beneficial therapeutic effects in HNSCC, although the response rate to ICIs remains poor [15]. A lncRNA signature of tumor-infiltrating B lymphocytes was found to have potential applications in prognostic prediction and immunotherapy for bladder cancer based on computational recognition [40], and lncRNAs associated with tumor immune infiltration were identified as improving clinical outcomes and immunotherapy response in non-small-cell lung cancer based on comprehensive analysis [41]. Combinations of ceRNA with ICIs and TIICs for improving prognosis and immunotherapy response in HNSCC are limited. Therefore, it is crucial to establish predictive biomarkers for selecting patients who will be responsive to ICI therapy.

In the present study, we defined nine key ceRNAs (ANLN, CFL2, ITGA5, KDELC1, KIF23, NFIA, PTX3, RELT, and TMC7) and three immune cell subtypes (naïve B cells, Tregs, and neutrophils) using univariate Cox analysis, LASSO, multivariate Cox analysis, and independent prognostic analysis. Specifically, KDELC1, PTX3, and ITGA5 were found to be associated with poor prognosis in both the K-M survival analysis and the multivariate Cox analysis. ROC curve analysis was used to further evaluate both signatures and confirm their favorable predictive and prognostic abilities.
We identified a potential regulatory mechanism involving KCNQ1OT1 (lncRNA), miR-338-3p (miRNA)/miR29c-3p, ITGA5 (mRNA)/KDELC1, and naïve B cells/Tregs.

A previous report indicated that KCNQ1OT1 was associated with cell proliferation, apoptosis, prognosis, invasion, and metastasis in various cancers [10, 42-44]. Further, KCNQ1OT1 facilitated invasion and inhibited apoptosis in oral squamous cell carcinoma by regulating the miR-1855p/Rab14 axis [45]. miR-338-3p was downregulated in esophageal squamous cell carcinoma and could act as a tumor suppressor [46]. In addition, miR-338-3p was shown to inhibit colorectal carcinoma cell invasion and migration by targeting smoothened [47] and was associated with favorable prognosis in urothelial carcinoma of the bladder [48]. Inhibition of miR-29c-3p was found to be associated with poor prognosis in patients with laryngeal squamous cell carcinoma [49]. ITGA5 has essential roles in tumorigenesis, migration, and invasion in various cancer types [10]. Studies have shown that ITGA5 is highly expressed in HNSCC, where its high expression is significantly associated with poor survival [50]. Overexpression of PTX3 has been related to poor prognosis in pancreatic cancer and is linked to more advanced stages of the disease [51]. Evidence also suggests that PTX3 promotes metastasis of cervical cancer and EGF-induced metastasis of HNSCC through upregulation of MMP-2 and MMP-9 [52]. Our results are in accordance with these findings and suggest that the KCNQ1OT1/miR338-3p/ITGA5 and KCNQ1OT1/miR-29c-3p/KDELC1 axes 


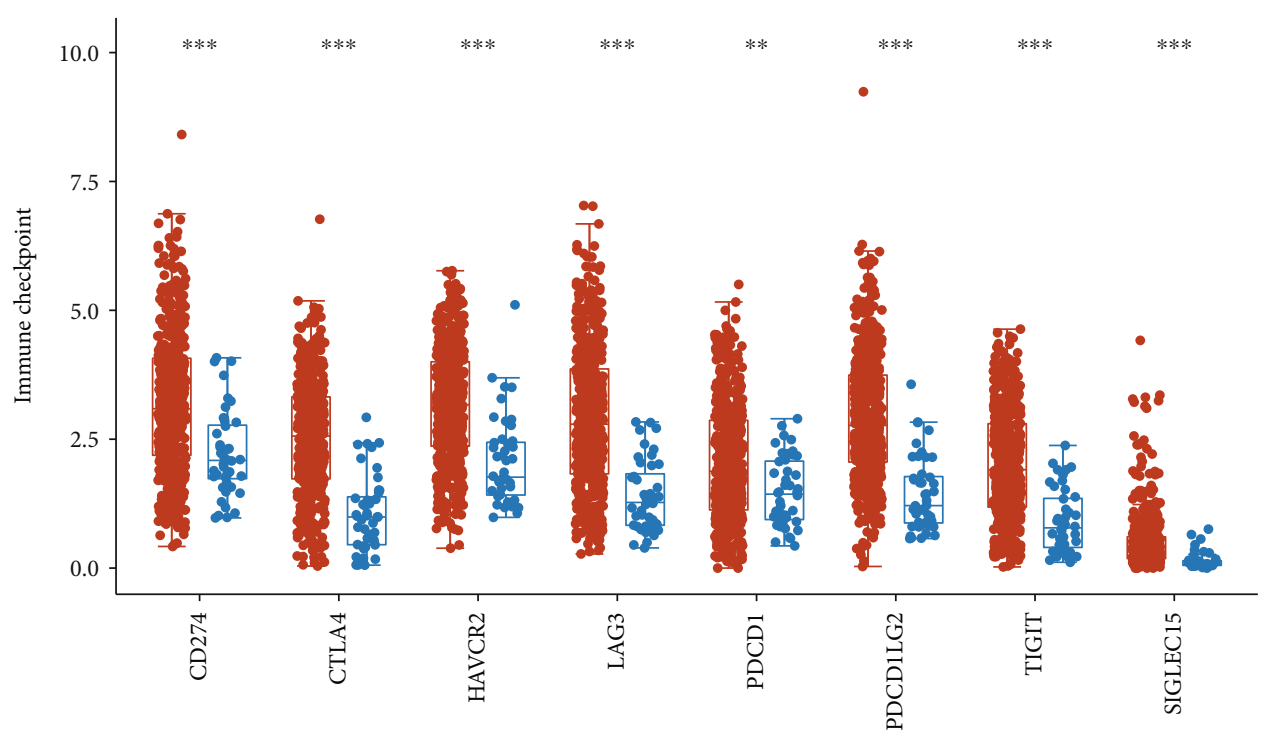

Group

Tumor

Normal

(a)

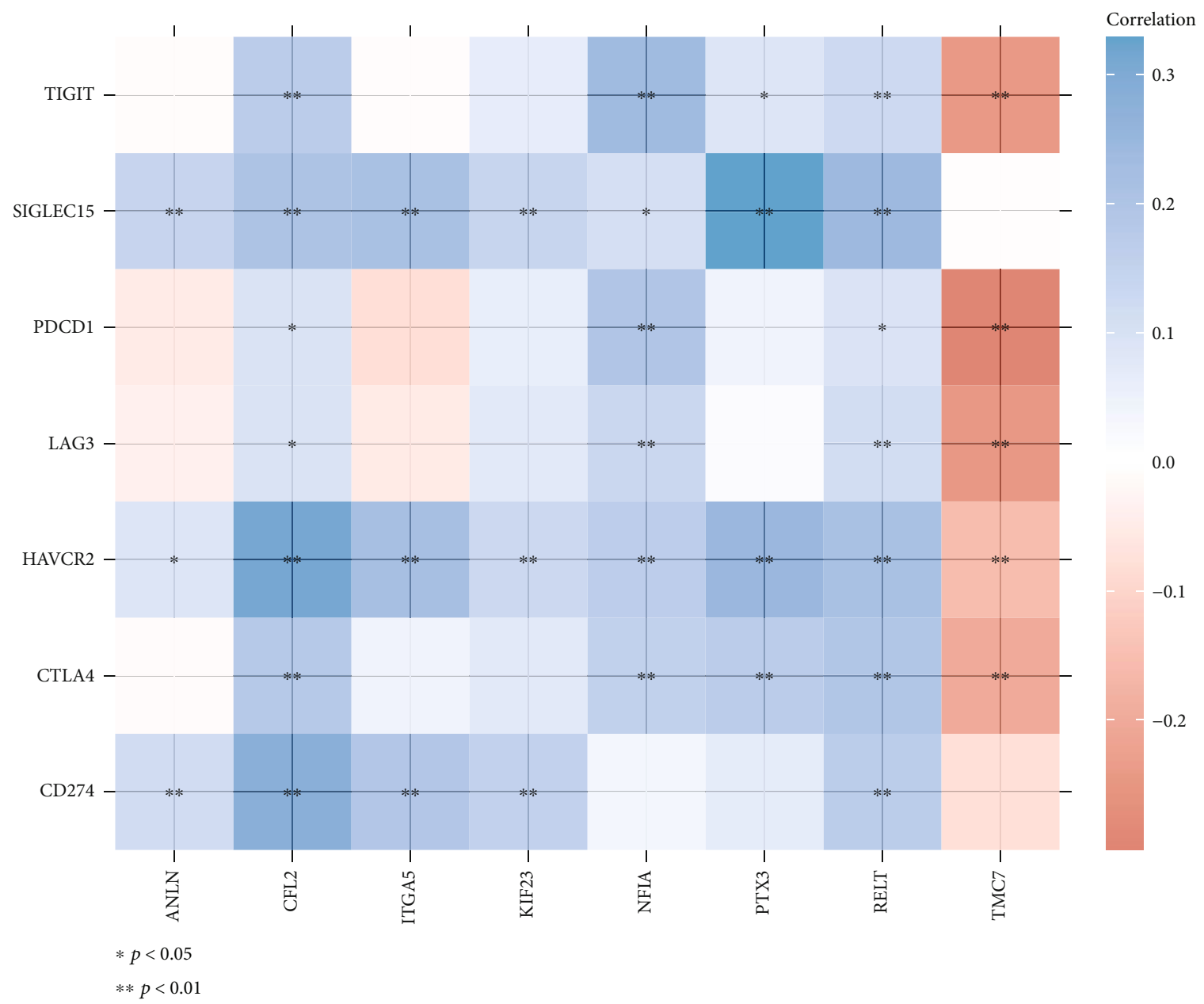

(b)

FIgURE 7: Continued. 


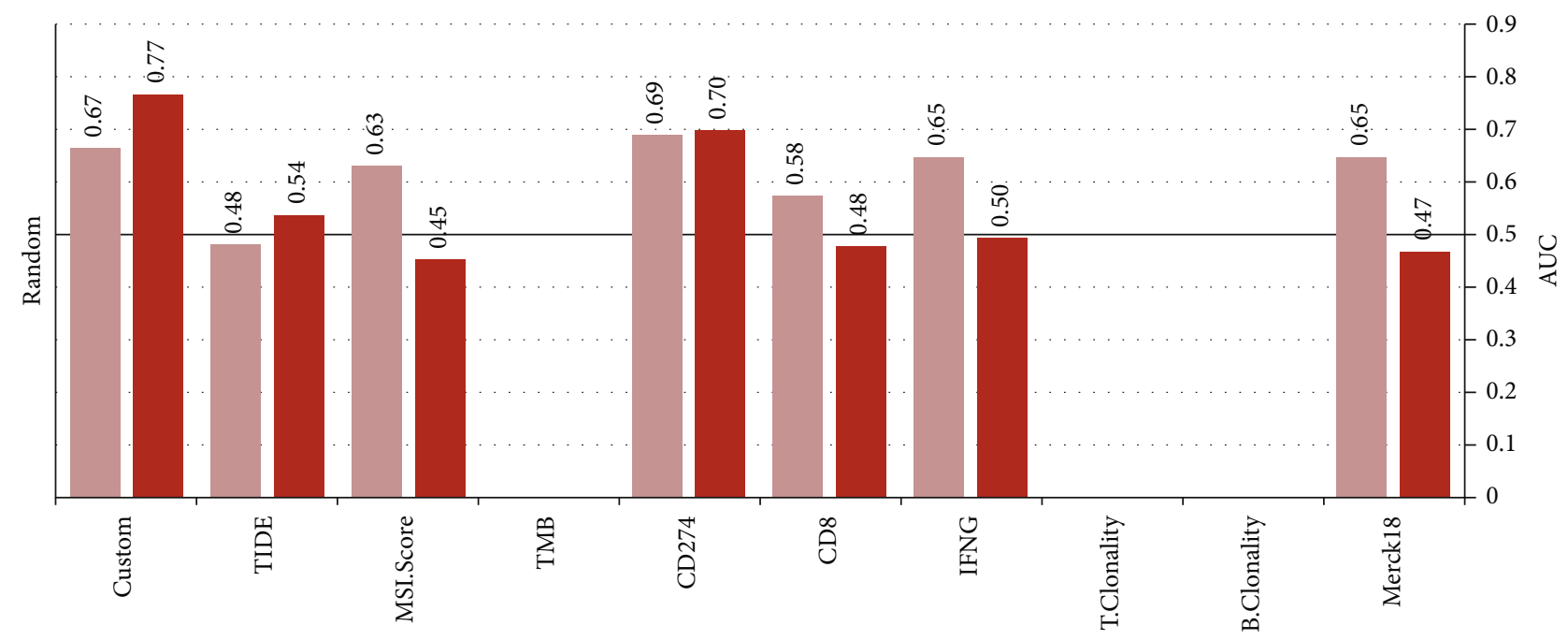

- Uppaluri2020_PD1_HNSC_Pre

$\mathrm{Pos}=8, \mathrm{Neg}=15$

- Uppaluri2020_PD1_HNSC_Post

Pos $=9, \mathrm{Neg}=13$

(c)

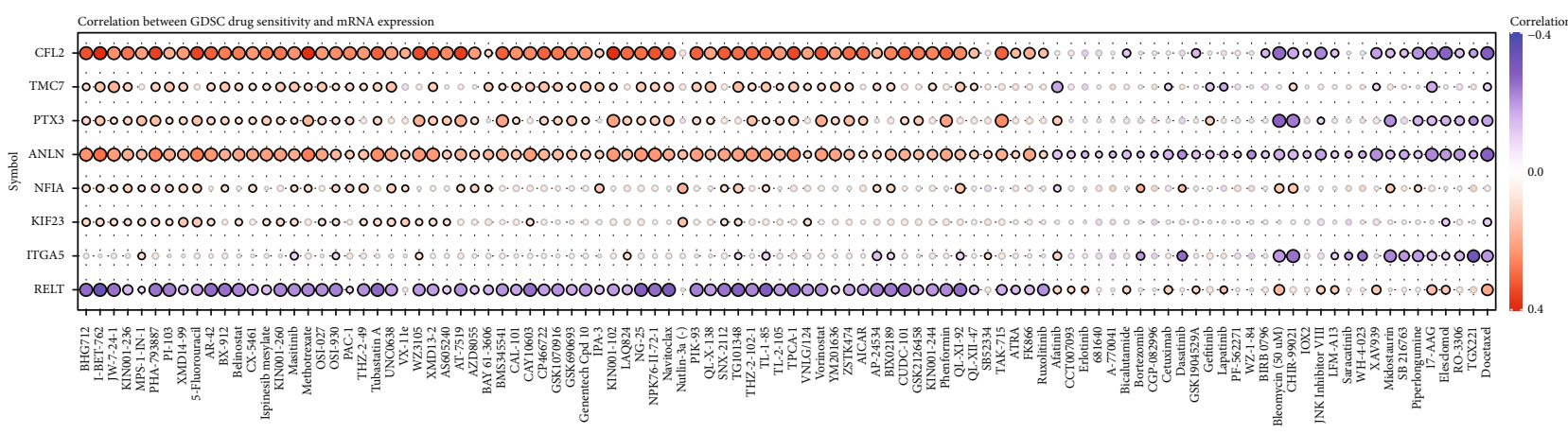

Drug

FDR
G $<0.05$

(d)

FIGURE 7: Key ceRNA expression correlates with immunotherapy response and drug sensitivity. (a) Differences in expression of tumorrelated immunosuppressive molecules in HNSCC; ${ }^{*} P<0.05,{ }^{* *} P<0.01$, and ${ }^{* * *} P<0.001$. (b) Correlations between key ceRNAs and IC molecules. The horizontal and vertical coordinates represent genes. Different colors represent correlation coefficients (blue, positive correlation and red, negative correlation); the darker the color, the stronger the correlation; ${ }^{*} P<0.05$ and ${ }^{* *} P<0.01$. (c) Comparison of AUC values between the custom biomarker and other published biomarkers in predicting anti-PD1 response. (d) Correlation analysis of drug sensitivity and expression of key ceRNAs.

TABLE 2: Survival analysis for major target ceRNAs using LOGpc.

\begin{tabular}{lccccc}
\hline Symbol & Dataset & HR & $95 \%$ CI & $P$ value & Prognostic \\
\hline CFL2 & TCGA & 1.3608 & $1.0137-1.8268$ & 0.0403 & 0.0038 \\
ITGA5 & GSE31056 & 2.6804 & $1.3752-5.2245$ & 0.0067 & Poor \\
ITGA5 & TCGA & 1.4878 & $1.1162-1.9832$ & 0.0061 & 0.0262 \\
KDELC1 & TCGA & 1.4946 & $1.1212-1.9922$ & 0.0068 & Poor \\
KDELC1 & GSE31056 & 2.1694 & $1.0960-4.2940$ & $<0.0001$ & Poor \\
NFIA & GSE65858 & 0.4627 & $0.2648-0.8086$ & 0.0426 & 0.0418 \\
PTX3 & GSE31056 & 3.9765 & $2.0435-7.7379$ & $1.0103-1.8320$ & Poor \\
PTX3 & TCGA & 1.3605 & $1.0113-1.8165$ & & Poor \\
RELT & TCGA & 1.3554 & &
\end{tabular}




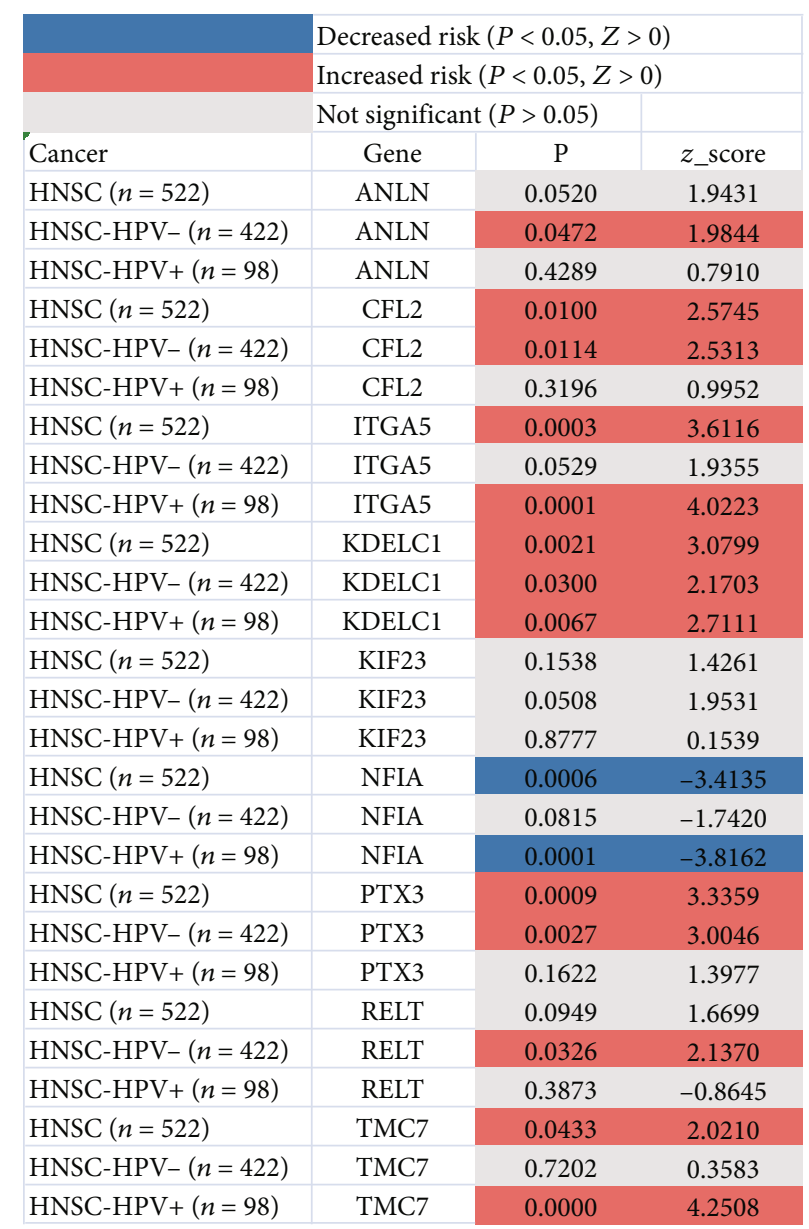

(a)

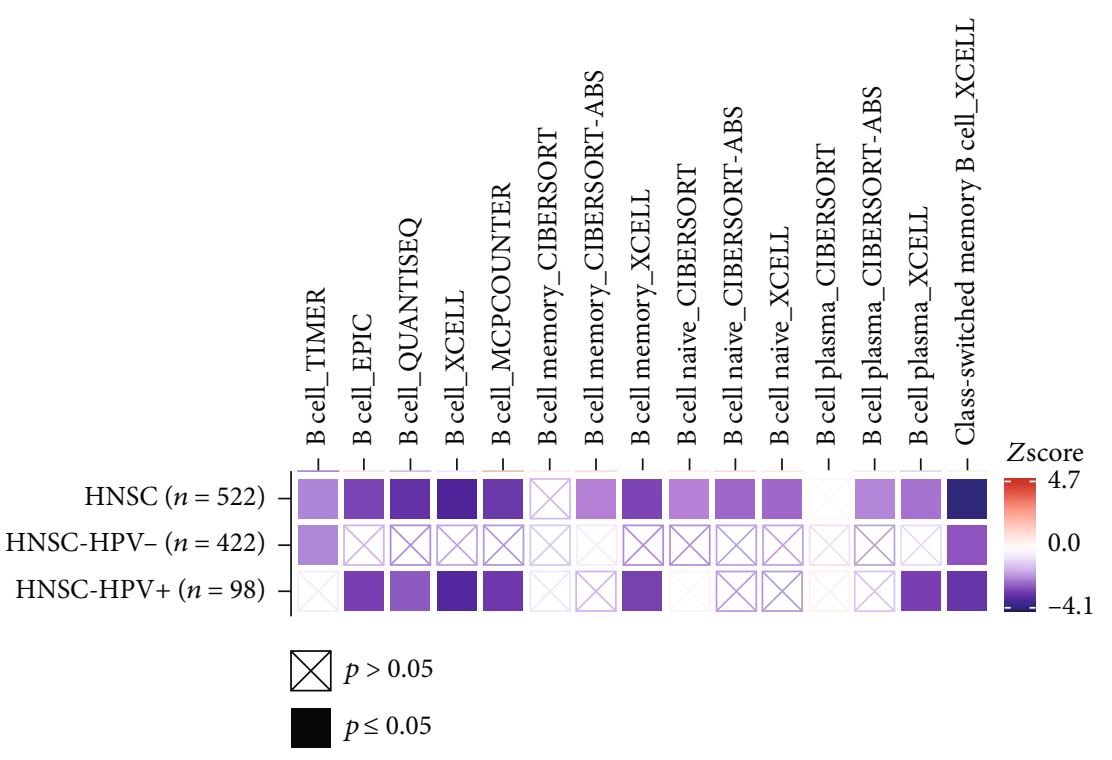

(b)

Figure 8: Continued. 


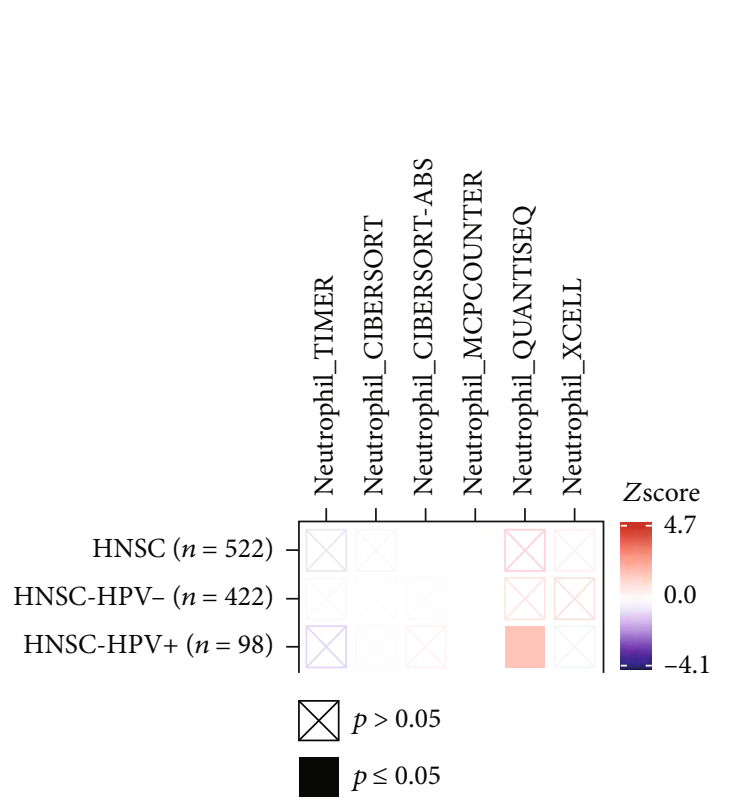

(c)

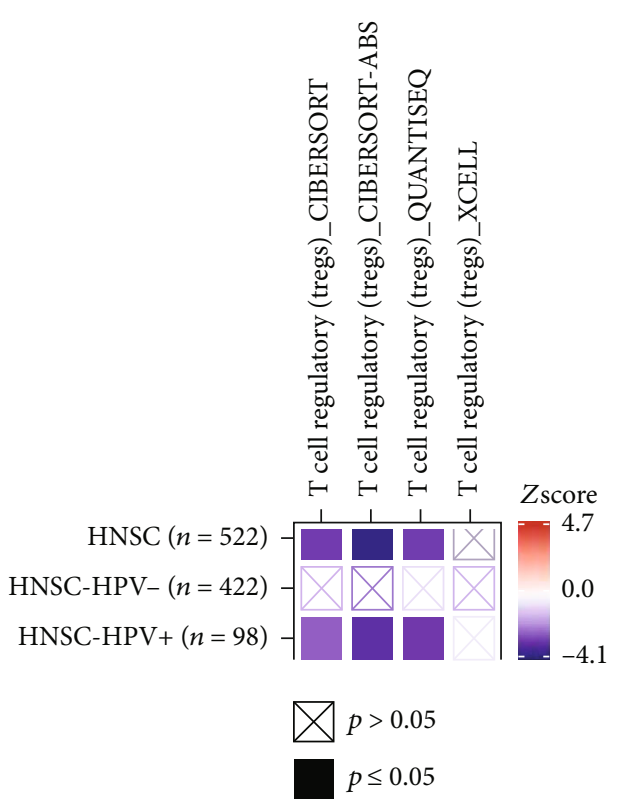

(d)

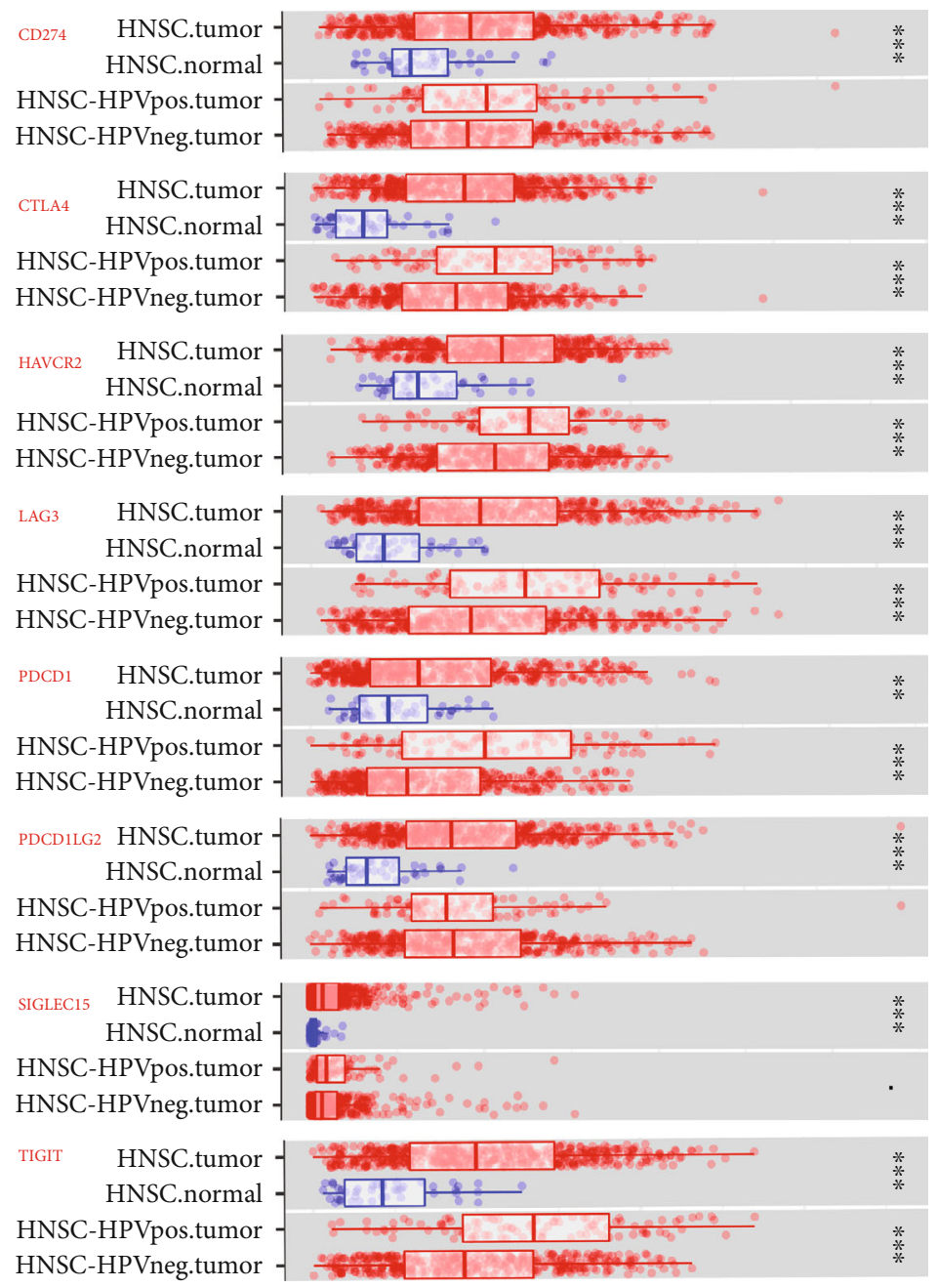

(e)

Figure 8: Continued. 
ANLN

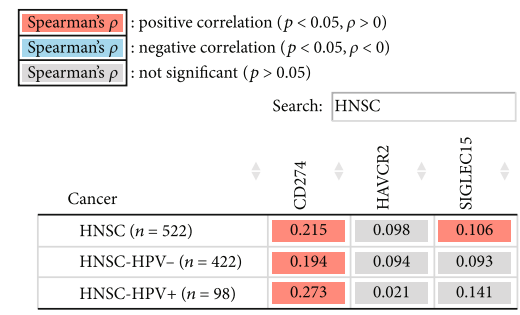

CFL2

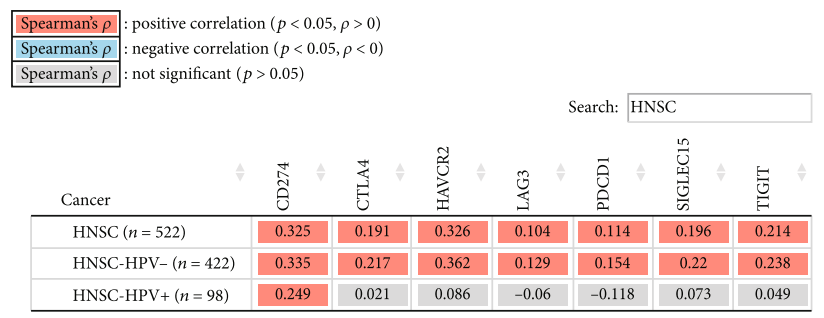

ITGA5

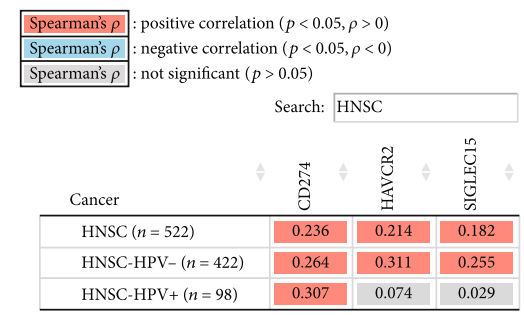

KIF23

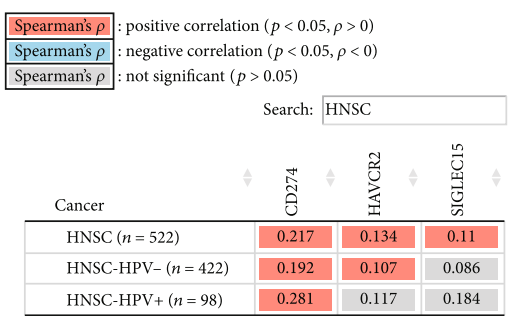

NFIA

\begin{tabular}{|l|l|l|}
\hline Spearman's $\rho$ & positive correlation $(p<0.05, \rho>0)$ \\
\cline { 1 - 2 } Spearman's $\rho$ & : negative correlation $(p<0.05, \rho<0)$ \\
\hline Spean & n
\end{tabular}

\begin{tabular}{ll}
\hline Spearman's $\rho$ & : not significant $(p>0.05)$
\end{tabular}

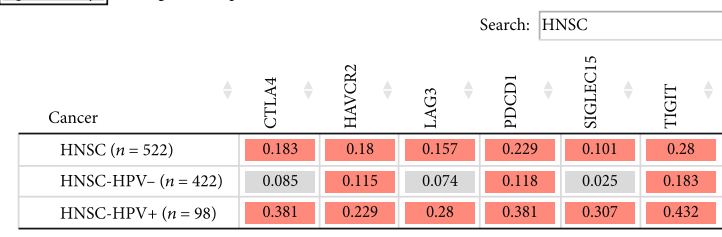

PTX3

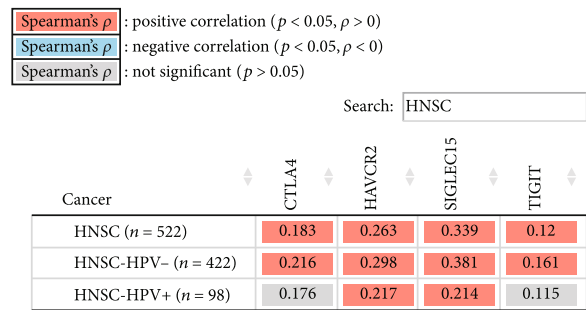

RELT

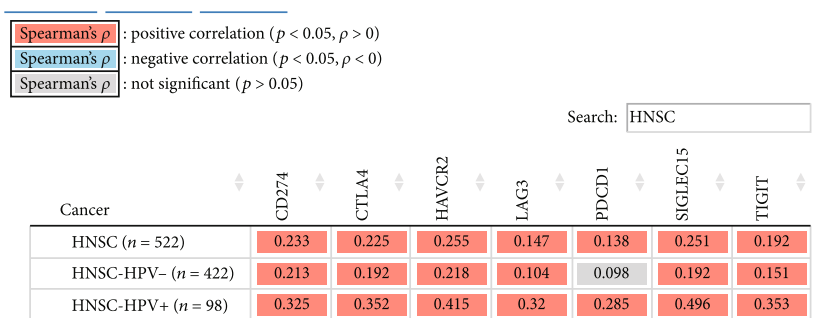

TMC7

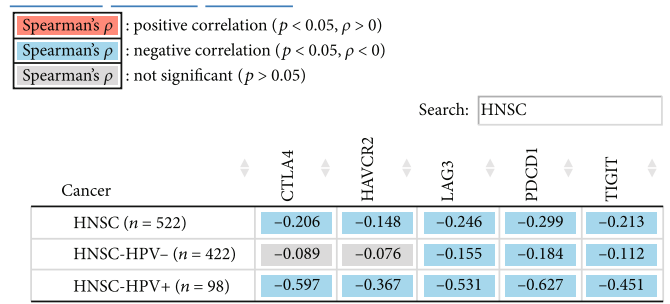

(f)

Figure 8: Validation of the prognostic value of identified biomarkers and associations between key ceRNAs and IC genes. (a) Confirmation of prognostic value of key ceRNAs in HNSCC with different HPV statuses via the TIMER 2.0 database. (b-d) Analysis of associations between key immune cells and OS in HNSCC patients with different HPV statuses based on Cox regression analysis using the TIMER 2.0 database (http://timer.cistrome.org/). (e) Differences in expression of IC genes with different HPV statuses in HNSCC using TIMER 2.0. (f) Exploration of the associations between key ceRNAs and IC molecules with different HPV statuses in HNSCC using TIMER 2.0.

have important roles in determining the prognosis of HNSCC patients.

The associations among the nine key ceRNA signatures, three immune cell signatures, and different clinicopathological features was assessed. The results showed that ANLN, KIF23, and ITGA5 expression was significantly correlated with grade. Increased KDELC1 and PTX3 expression was related to advanced stage, whereas overexpression of NFIA was correlated with low tumor stage (Figures 6(a)-6(f)). A recent study showed that the $\mathrm{C} 1$ and $\mathrm{C} 2$ subtypes were enriched in HNSCC and were associated with less favorable outcomes. The C3 subtype had the most favorable prognosis, whereas the C4 and C6 subtypes had the worst prognoses [53]. In our study, we observed increased expression of ANLN and KIF23 in subtypes C1 and C2, whereas KDELC1 showed higher expression in the C1, C2, and C6 subtypes, indicating that it may be associated with worse survival. NFIA showed increased expression in subtype C3 and decreased expression in $\mathrm{C} 4$, which indicated that it could predict better survival (Figures 6(g) $6(\mathrm{j})$ ). 
TABLE 3: Associations between gene expression and therapy outcome in clinical studies of IC blockade.

\begin{tabular}{|c|c|c|c|c|c|}
\hline Gene & Cohort & Cancer & Subtype & Survival & Risk.adj \\
\hline \multirow{2}{*}{ ANLN } & Riaz2017_PD1 & Melanoma & Ipi_Prog & OS & 2.025 \\
\hline & Riaz2017_PD1 & Melanoma & Ipi_naive & PFS & -3.136 \\
\hline CFL2 & Liu2019_PD1 & Melanoma & Ipi_naive & OS & 2.489 \\
\hline \multirow{2}{*}{ ITGA5 } & Mariathasan2018_PDL1 & Bladder & mUC & OS & 3.52 \\
\hline & Miao2018_ICB & Kidney & Clear & PFS & -3.173 \\
\hline KIF23 & Riaz2017_PD1 & Melanoma & Ipi_Prog & OS & 2.802 \\
\hline \multirow{2}{*}{ NFIA } & Mariathasan2018_PDL1 & Bladder & $\mathrm{mUC}$ & OS & 2.987 \\
\hline & Zhao2019_PD1 & Glioblastoma & Pre & PFS & -2.336 \\
\hline PTX3 & Riaz2017_PD1 & Melanoma & Ipi_naive & OS & 2.467 \\
\hline \multirow{2}{*}{ RELT } & Mariathasan2018_PDL1 & Bladder & mUC & OS & 2.505 \\
\hline & Liu2019_PD1 & Melanoma & Ipi_naive & PFS & -2.32 \\
\hline \multirow{2}{*}{ TMC7 } & Hugo2016_PD1 & Melanoma & & OS & 2.27 \\
\hline & Lauss2017_ACT & Melanoma & & OS & -2.528 \\
\hline
\end{tabular}

Recently, Walter et al. [13] reported that the MS subtype was associated with distant metastases, and that treatment with EGFR inhibitors was less likely to be effective in the AT subtype. Our results indicated that CFL2, ITGA5, KDELC1, and PTX3 were highly expressed in the MS subtype. Higher NFIA expression was associated with the AT subtype (Figures 6(k)-6(q)). These studies further support our finding that NFIA expression was correlated with favorable outcomes whereas higher expression of the other ceRNAs was associated with unfavorable outcomes.

Previous studies have revealed that naïve $B$ cells and Tregs are indicators of better survival, whereas neutrophils have been associated with HPV positivity and poorer outcomes [7, 10, 54]. Higher Treg infiltration was found to be correlated with better prognosis and longer survival in HPV-positive patients compared with HPV-negative HNSCC patients [55]. By contrast, naïve B cells could be correlated with tumorigenesis and progression of HNSCC [10]. Neutrophils induce tumor progression and promote tumor cell migration in HNSCC; therefore, neutrophil infiltration represents a risk factor. $[56,57]$. Our integrated analyses showed that three immune cell subtypes (naive B cells, Tregs, and neutrophils) could be used as prognosis-related biomarkers in HNSCC. Naïve B cells and Tregs were associated with favorable prognosis, whereas neutrophils represented an unfavorable prognostic marker in HNSCC, especially in HPV-positive HNSCC patients (Figures 4(e) and $8(\mathrm{~b})-8(\mathrm{~d}))$. ROC analysis showed that the key immune cell-related model constructed in this study could predict the prognosis of patients with HNSCC. As shown in Figures 5(j)-5(l), higher levels of naïve $B$ cells and Tregs were related to lower risk scores $(P=0.0085$ and $P=4 e-9$ ). These results further suggested that these immune cell types (naïve B cells, Tregs, and neutrophils) were related to prognosis. Our results are consistent with those of previous studies, and our immune cell-related model could be used as an independent factor to assess prognosis in HNSCC.

Finally, we found that eight IC genes exhibited higher expression in tumor tissues and were correlated with key ceR-
NAs in HNSCC with different HPV statuses (Figures 7(a) and 7(b) and 8(e) and 8(f)). Our identified biomarker could more effectively predict anti-PD1 response compared with published biomarkers (Figure $7(\mathrm{c})$ ). Previous studies have reported that HPV-positive HNCs expressed high levels of multiple $\mathrm{T}$ cell exhaustion markers, including LAG3, PD1, TIGIT, and TIM3; the high expression of these markers was correlated with improved survival in HPV-positive HNC patients $[58,59]$. Based on these studies and our findings, HNSCC may exhibit strong beneficial responses to immunotherapy and high expression levels of these key genes may contribute to predicting improved survival of HNSCC patients, especially in cases of HPV-positive HNSCC. We also observed that eight key genes were associated with treatment response to most drugs, suggesting the potential of these molecules as therapeutic drug targets in HNSCC (Figure $7(\mathrm{~d})$ ). These results provide a scientific rationale for potentially combining ICI therapy with inhibitors of the eight key genes identified in this study to improve treatment efficacy for HNSCC patients.

\section{Conclusions}

We identified nine key ceRNAs and three immune cellrelated signatures as potential biomarkers for predicting the prognosis of HNSCC. In addition, the KCNQ1OT1 (lncRNA), miR-338-3p (miRNA)/miR-29c-3p, ITGA5 (mRNA)/KDELC1, and naïve B cell/Treg axes may be linked to prognosis of HNSCC. As key IC-related members of ceRNAs may be associated with response to ICI therapy, combining ICI with inhibitors of these eight key genes may contribute to improving treatment efficacy in HNSC patients. However, additional clinical data and experiments are required to confirm the prognostic value of these signatures and their potential associations with ICI immunotherapy outcomes in patients with HNSCC. We will perform further experiments to confirm the current research results. 


\section{Data Availability}

The gene expression RNA-sequencing data and clinical information of HNSCC in the current study were obtained from TCGA data portal (https://portal.gdc.cancer.gov/).

\section{Conflicts of Interest}

The authors declare that there is no conflict of interest regarding the publication of this manuscript.

\section{Authors' Contributions}

Ya Guo conceived and designed the article, performed data analysis, and wrote the main text of the article. Wei Kang Pan reviewed the manuscript. Zhong Wei Wang performed language editing. Wang $\mathrm{Hui} \mathrm{Su}$ and Kun $\mathrm{Xu}$ prepared Figures 1-4. Hui Jia and Jing Chen prepared Figures 5-8 and tables. Ya Guo and Wei Kang Pan contributed equally to this work and are co-first authors.

\section{Acknowledgments}

This work was supported by the Shaanxi Provincial Natural Science Foundation (grant number 2017JQ8057). We thank Zhong Guo Liang for bioinformatics-related technical support.

\section{Supplementary Materials}

Supplementary 1. Additional file 1. Original source data: online web service.

Supplementary 2. Additional file 2. Original source data: related $\mathrm{R}$ software.

\section{References}

[1] L. Zhang, B. Li, Y. Peng et al., "The prognostic value of TMB and the relationship between TMB and immune infiltration in head and neck squamous cell carcinoma: a gene expression-based study," Oral Oncology, vol. 110, article 104943, 2020.

[2] Y. Zhang, A. Lin, Y. Li et al., "Age and mutations as predictors of the response to immunotherapy in head and neck squamous cell cancer," Frontiers in cell and developmental biology, vol. 8, article 608969, 2020.

[3] J. Li, R. M. Srivastava, A. Ettyreddy, and R. L. Ferris, "Cetuximab ameliorates suppressive phenotypes of myeloid antigen presenting cells in head and neck cancer patients," Journal for Immunotherapy of Cancer, vol. 3, no. 1, p. 54, 2015.

[4] E. E. W. Cohen, R. B. Bell, C. B. Bifulco et al., "The Society for Immunotherapy of Cancer consensus statement on immunotherapy for the treatment of squamous cell carcinoma of the head and neck (HNSCC)," Journal for Immunotherapy of Cancer, vol. 7, no. 1, p. 184, 2019.

[5] Y. Pan, G. Liu, D. Wang, and Y. Li, "Analysis of lncRNAmediated ceRNA crosstalk and identification of prognostic signature in head and neck squamous cell carcinoma," Frontiers in Pharmacology, vol. 10, p. 150, 2019.

[6] A. Jiang, N. Liu, S. Bai et al., "The construction and analysis of tumor-infiltrating immune cells and ceRNA networks in bladder cancer," Frontiers in Genetics, vol. 11, article 605767, 2020.

[7] J. Song, Z. Deng, J. Su, D. Yuan, J. Liu, and J. Zhu, "Patterns of immune infiltration in HNC and their clinical implications: a gene expression-based study," Frontiers in Oncology, vol. 9, p. $1285,2019$.

[8] X. Zhang, M. Shi, T. Chen, and B. Zhang, "Characterization of the immune cell infiltration landscape in head and neck squamous cell carcinoma to aid immunotherapy," Molecular therapy Nucleic acids, vol. 22, pp. 298-309, 2020.

[9] X.-N. Fang, M. Yin, H. Li et al., "Comprehensive analysis of competitive endogenous RNAs network associated with head and neck squamous cell carcinoma," Scientific Reports, vol. 8, no. 1, article 10544, 2018.

[10] H. Zhou, Y. He, L. Li, C. Wu, and G. Hu, "Identification novel prognostic signatures for head and neck squamous cell carcinoma based on ceRNA network construction and immune infiltration analysis," International Journal of Medical Sciences, vol. 18 , no. 5, pp. 1297-1311, 2021.

[11] R. L. Ferris, "Immunology and immunotherapy of head and neck cancer," Journal of Clinical Oncology: Official Journal of the American Society of Clinical Oncology, vol. 33, no. 29, pp. 3293-3304, 2015.

[12] X. Xu, J. Li, J. Zou et al., "Association of germline variants in natural killer cells with tumor immune microenvironment subtypes, tumor-infiltrating lymphocytes, immunotherapy response, clinical outcomes, and cancer risk," JAMA network open, vol. 2, no. 9, article e199292, 2019.

[13] V. Walter, X. Yin, M. D. Wilkerson et al., "Molecular subtypes in head and neck cancer exhibit distinct patterns of chromosomal gain and loss of canonical cancer genes," PloS one, vol. 8, no. 2, article e56823, 2013.

[14] S. Farkona, E. P. Diamandis, and I. M. Blasutig, "Cancer immunotherapy: the beginning of the end of cancer?," BMC Medicine, vol. 14, no. 1, p. 73, 2016.

[15] L. Yi, G. Wu, L. Guo, X. Zou, and P. Huang, "Comprehensive analysis of the PD-L1 and immune infiltrates of mA RNA methylation regulators in head and neck squamous cell carcinoma," Molecular therapy Nucleic acids, vol. 21, pp. 299-314, 2020.

[16] G. Wang, D. Xu, Z. Zhang et al., “The pan-cancer landscape of crosstalk between epithelial-mesenchymal transition and immune evasion relevant to prognosis and immunotherapy response," NPJ precision oncology, vol. 5, no. 1, p. 56, 2021.

[17] B. Feng and J. Hess, "Immune-related mutational landscape and gene signatures: prognostic value and therapeutic impact for head and neck cancer," Cancers, vol. 13, no. 5, p. 1162, 2021.

[18] Y. Yao, Z. Yan, S. Lian et al., "Prognostic value of novel immune-related genomic biomarkers identified in head and neck squamous cell carcinoma," Journal for immunotherapy of cancer, vol. 8, no. 2, article e000444, 2020.

[19] C. Ili, K. Buchegger, H. Demond et al., "Landscape of genomewide DNA methylation of colorectal cancer metastasis," Cancers, vol. 12, no. 9, p. 2710, 2020.

[20] E. Pérez-Riesgo, L. Gutiérrez, D. Ubierna et al., "Transcriptomic analysis of calcium remodeling in colorectal cancer," International journal of molecular sciences, vol. 18, no. 5, p. 922, 2017.

[21] Y. Zhu, Y. Bian, Q. Zhang et al., "Construction and analysis of dysregulated lncRNA- associated ceRNA network in colorectal 
cancer," Journal of Cellular Biochemistry, vol. 120, no. 6, pp. 9250-9263, 2019.

[22] P. Shannon, A. Markiel, O. Ozier et al., "Cytoscape: a software environment for integrated models of biomolecular interaction networks," Genome Research, vol. 13, no. 11, pp. 24982504, 2003.

[23] R. Huang, J. Wu, Z. Zheng et al., "The construction and analysis of ceRNA network and patterns of immune infiltration in mesothelioma with bone metastasis," Frontiers in Bioengineering and Biotechnology, vol. 7, p. 257, 2019.

[24] K. Lin, L.-J. Song, J. Ma, T.-S. Zhang, D.-Y. You, and Y.-W. He, "Identification of cancer hallmark-associated gene and lncRNA cooperative regulation pairs and dictate lncRNA roles in oral squamous cell carcinoma," Journal of Cellular and Molecular Medicine, vol. 24, no. 9, pp. 5213-5223, 2020.

[25] Y. Guo, P. T. Yang, Z. W. Wang, K. Xu, W. H. Kou, and H. Luo, "Identification of three autophagy-related long noncoding RNAs as a novel head and neck squamous cell carcinoma prognostic signature," Frontiers in Oncology, vol. 10, article 603864, 2021.

[26] S. Liu, A. Song, X. Zhou et al., "ceRNA network development and tumour-infiltrating immune cell analysis of metastatic breast cancer to bone," Journal of bone oncology, vol. 24, article 100304, 2020.

[27] J. Lin, M. Yu, X. Xu et al., "Identification of biomarkers related to CD8 T cell infiltration with gene co-expression network in clear cell renal cell carcinoma," Aging, vol. 12, no. 4, pp. 3694-3712, 2020.

[28] P. Jiang, S. Gu, D. Pan et al., "Signatures of T cell dysfunction and exclusion predict cancer immunotherapy response," Nature Medicine, vol. 24, no. 10, pp. 1550-1558, 2018.

[29] C.-J. Liu, F.-F. Hu, M. Xia, L. Han, Q. Zhang, and A. Guo, "GSCALite: a web server for gene set cancer analysis," Bioinformatics (Oxford, England), vol. 34, no. 21, pp. 3771-3772, 2018.

[30] W. Yang, J. Soares, P. Greninger et al., "Genomics of Drug Sensitivity in Cancer (GDSC): a resource for therapeutic biomarker discovery in cancer cells," Nucleic Acids Research, vol. 41, no. Database issue, pp. D955-D961, 2013.

[31] G. Wu, Y. Xu, N. Ruan et al., "Genetic alteration and clinical significance of SUMOylation regulators in multiple cancer types," Journal of Cancer, vol. 11, no. 23, pp. 6823-6833, 2020.

[32] H. Zheng, G. Zhang, L. Zhang et al., "Comprehensive review of web servers and bioinformatics tools for cancer prognosis analysis," Frontiers in Oncology, vol. 10, p. 68, 2020.

[33] B. Hu, X.-B. Yang, and X.-T. Sang, "Development of an immune-related prognostic index associated with hepatocellular carcinoma," Aging, vol. 12, no. 6, pp. 5010-5030, 2020.

[34] T. Li, J. Fu, Z. Zeng et al., "TIMER2.0 for analysis of tumorinfiltrating immune cells," Nucleic Acids Research, vol. 48, no. W1, pp. W509-W514, 2020.

[35] C. R. Leemans, B. J. M. Braakhuis, and R. H. Brakenhoff, “The molecular biology of head and neck cancer," Nature Reviews Cancer, vol. 11, no. 1, pp. 9-22, 2011.

[36] Z. X. Li, Z. Q. Zheng, Z. H. Wei et al., "Comprehensive characterization of the alternative splicing landscape in head and neck squamous cell carcinoma reveals novel events associated with tumorigenesis and the immune microenvironment," Theranostics, vol. 9, no. 25, pp. 7648-7665, 2019.

[37] A.-M. Jiang, M.-D. Ren, N. Liu et al., "Tumor mutation burden, immune cell infiltration, and construction of immunerelated genes prognostic model in head and neck cancer,"
International Journal of Medical Sciences, vol. 18, no. 1, pp. 226-238, 2021.

[38] Z. Wang, H. Yuan, J. Huang et al., "Prognostic value of immune-related genes and immune cell infiltration analysis in the tumor microenvironment of head and neck squamous cell carcinoma," Head \& Neck, vol. 43, no. 1, pp. 182-197, 2021.

[39] Y. Chen, Y. Li, R. Narayan, A. Subramanian, and X. Xie, "Gene expression inference with deep learning," Bioinformatics (Oxford, England), vol. 32, no. 12, pp. 1832-1839, 2016.

[40] M. Zhou, Z. Zhang, S. Bao et al., "Computational recognition of lncRNA signature of tumor-infiltrating B lymphocytes with potential implications in prognosis and immunotherapy of bladder cancer," Briefings in Bioinformatics, vol. 22, no. 3, 2021.

[41] J. Sun, Z. Zhang, S. Bao et al., "Identification of tumor immune infiltration-associated lncRNAs for improving prognosis and immunotherapy response of patients with non-small cell lung cancer," Journal for immunotherapy of cancer, vol. 8, no. 1, article e000110, 2020.

[42] Y. Zhu, Y. Shen, R. Chen et al., "KCNQ1OT1 lncRNA affects the proliferation, apoptosis, and chemoresistance of small cell lung cancer cells via the JAK2/STAT3 axis," Annals of translational medicine, vol. 9, no. 10, p. 891, 2021.

[43] H. Hao, H. Chen, L. Xie, H. Liu, and D. Wang, "LncRNA KCNQ1OT1 promotes proliferation, invasion and metastasis of prostate cancer by regulating miR-211-5p/CHI3L1 pathway," Oncotargets and Therapy, vol. 14, pp. 1659-1671, 2021.

[44] X. Zhong, X. Wen, L. Chen, N. Gu, X. Yu, and K. Sui, "Long non-coding RNA KCNQ1OT1 promotes the progression of gastric cancer via the miR-145-5p/ARF6 axis," The Journal of Gene Medicine, vol. 23, no. 5, article e3330, 2021.

[45] Q. Bao, X. Liao, R. Li, and N. Ding, "KCNQ1OT1 promotes migration and inhibits apoptosis by modulating miR-185-5p/ Rab14 axis in oral squamous cell carcinoma," Development, Growth \& Differentiation, vol. 61, no. 9, pp. 466-474, 2019.

[46] M. Yang, R. Liu, J. Sheng et al., "Differential expression profiles of microRNAs as potential biomarkers for the early diagnosis of esophageal squamous cell carcinoma," Oncology Reports, vol. 29, no. 1, pp. 169-176, 2013.

[47] Q. Xue, K. Sun, H.-J. Deng, S.-T. Lei, J.-Q. Dong, and G.-X. Li, "MicroRNA-338-3p inhibits colorectal carcinoma cell invasion and migration by targeting smoothened," Japanese Journal of Clinical Oncology, vol. 44, no. 1, pp. 13-21, 2014.

[48] T. Inamoto, H. Uehara, Y. Akao et al., "A panel of microRNA signature as a tool for predicting survival of patients with urothelial carcinoma of the bladder," Disease Markers, vol. 2018, Article ID 5468672, 6 pages, 2018.

[49] R. Fang, Y. Huang, J. Xie, J. Zhang, and X. Ji, “Downregulation of miR-29c-3p is associated with a poor prognosis in patients with laryngeal squamous cell carcinoma," Diagnostic Pathology, vol. 14, no. 1, p. 109, 2019.

[50] S. Kisoda, W. Shao, N. Fujiwara et al., "Prognostic value of partial EMT-related genes in head and neck squamous cell carcinoma by a bioinformatic analysis," Oral Diseases, vol. 26, no. 6, pp. 1149-1156, 2020.

[51] S. Kondo, H. Ueno, H. Hosoi et al., "Clinical impact of pentraxin family expression on prognosis of pancreatic carcinoma," British Journal of Cancer, vol. 109, no. 3, pp. 739-746, 2013.

[52] S.-H. Chan, J. P. Tsai, C.-J. Shen, Y.-H. Liao, and B. K. Chen, "Oleate-induced PTX3 promotes head and neck squamous cell 
carcinoma metastasis through the up-regulation of vimentin," Oncotarget, vol. 8, no. 25, pp. 41364-41378, 2017.

[53] V. Thorsson, D. L. Gibbs, S. D. Brown et al., "The immune landscape of cancer," Immunity, vol. 48, no. 4, pp. 812830.e14, 2018.

[54] J. Zhang, X. Zhong, H. Jiang et al., "Comprehensive characterization of the tumor microenvironment for assessing immunotherapy outcome in patients with head and neck squamous cell carcinoma," Aging, vol. 12, no. 22, pp. 22509-22526, 2020.

[55] X. Liu, J. Chen, W. Lu et al., "Systematic profiling of immune risk model to predict survival and immunotherapy response in head and neck squamous cell carcinoma," Frontiers in Genetics, vol. 11, article 576566, 2020.

[56] N. Borregaard, "Neutrophils, from marrow to microbes," Immunity, vol. 33, no. 5, pp. 657-670, 2010.

[57] C. A. Dumitru, H. Gholaman, S. Trellakis et al., "Tumorderived macrophage migration inhibitory factor modulates the biology of head and neck cancer cells via neutrophil activation," International Journal of Cancer, vol. 129, no. 4, pp. 859869, 2011.

[58] S. F. Gameiro, F. Ghasemi, J. W. Barrett et al., "Treatmentnaïve HPV+ head and neck cancers display a T-cell-inflamed phenotype distinct from their HPV- counterparts that has implications for immunotherapy," Oncoimmunology, vol. 7, no. 10, article e1498439, 2018.

[59] W. Qiang, Y. Dai, X. Xing, and X. Sun, "Identification and validation of a prognostic signature and combination drug therapy for immunotherapy of head and neck squamous cell carcinoma," Computational and Structural Biotechnology Journal, vol. 19, pp. 1263-1276, 2021. 